\title{
-
}

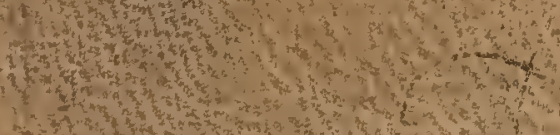

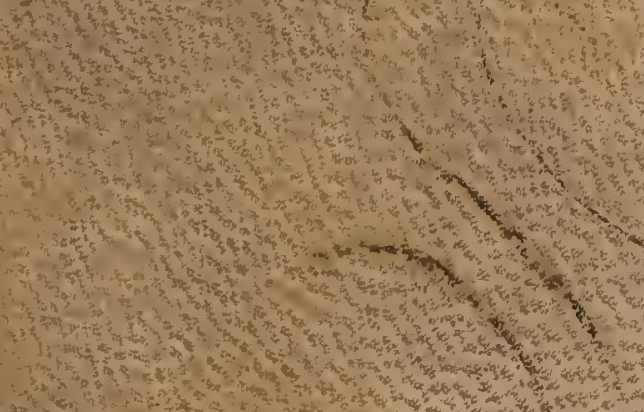

axas

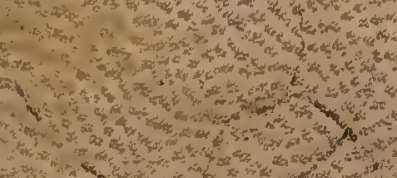

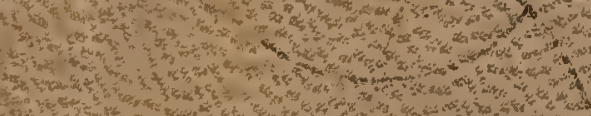

$+\sqrt{2}$

Hot

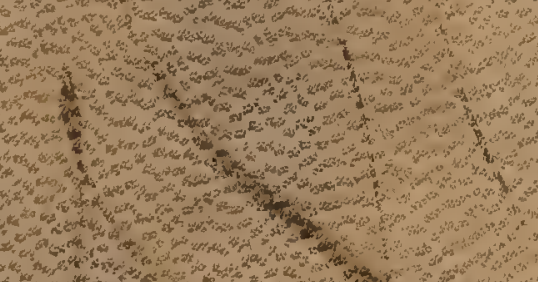

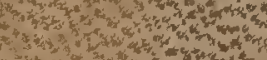

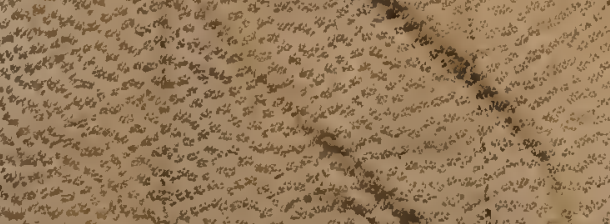

$3 x^{2}+x^{2} x^{2}$

-

-

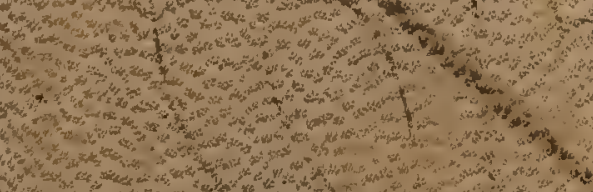

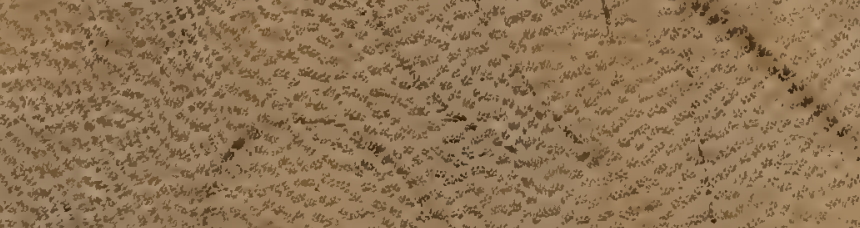

Hot
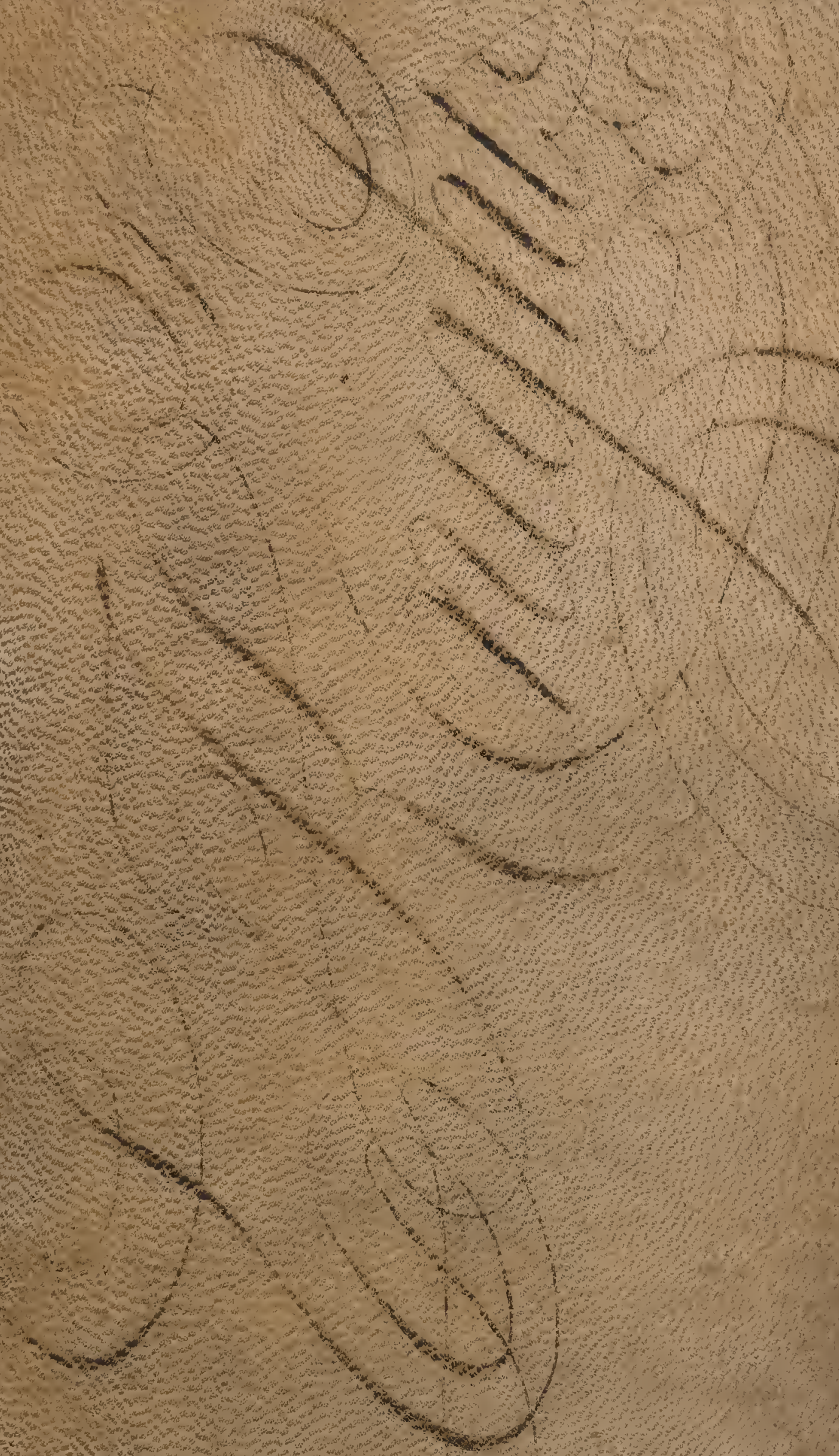


$$
110
$$

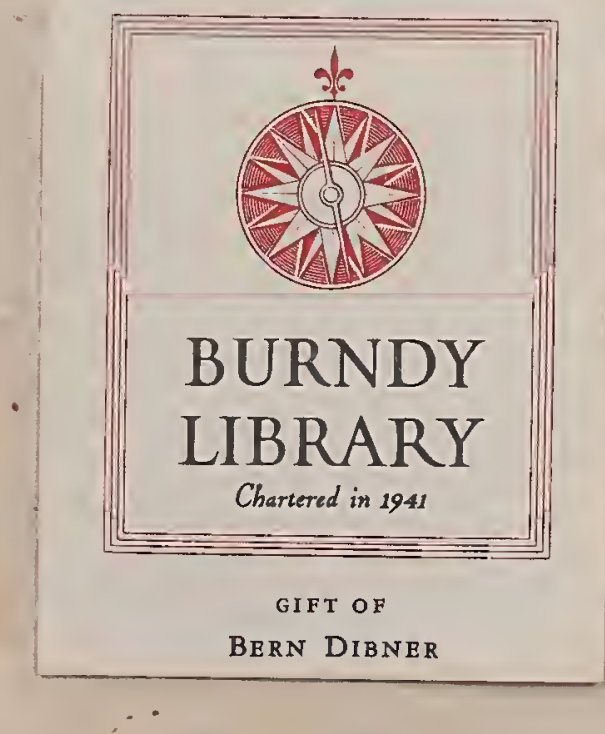




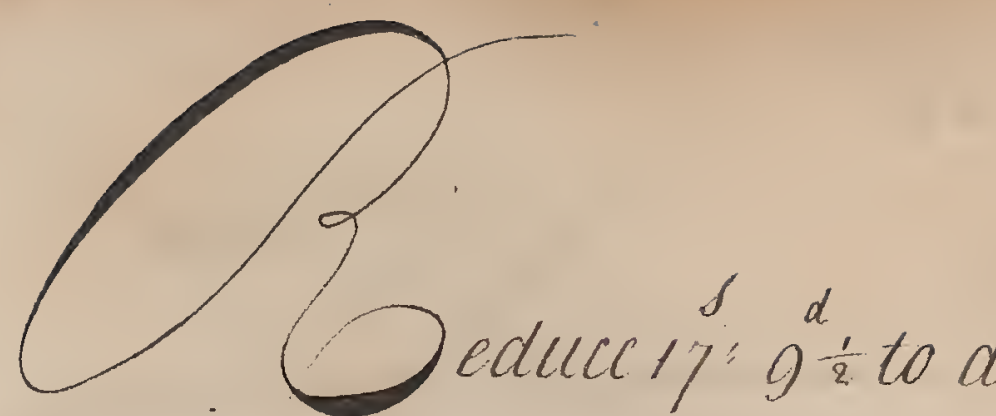

Dermal?

$\operatorname{lig} d \frac{d}{2}$

.85

$\frac{.039}{.009}$ answer to as Decimal?

$\int_{4} \cdot 15: 4 d$

4.75

$\frac{.017}{4.767}$ Answer

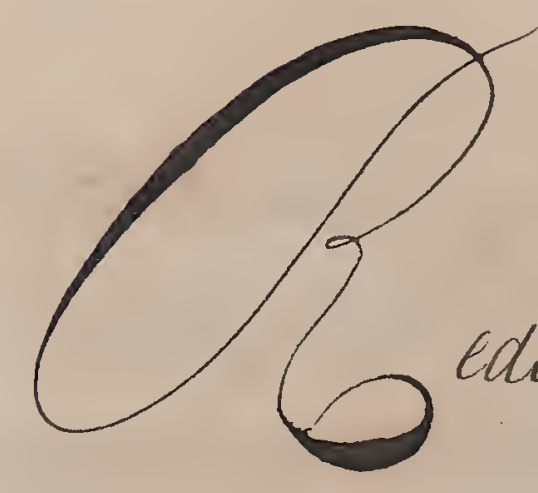

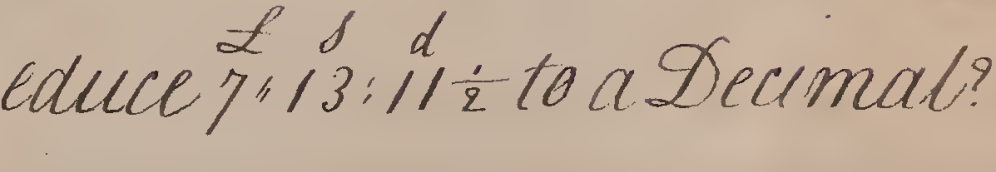

$$
\begin{aligned}
& 7.65 \\
& \frac{.047}{7.697} \text { answer }
\end{aligned}
$$




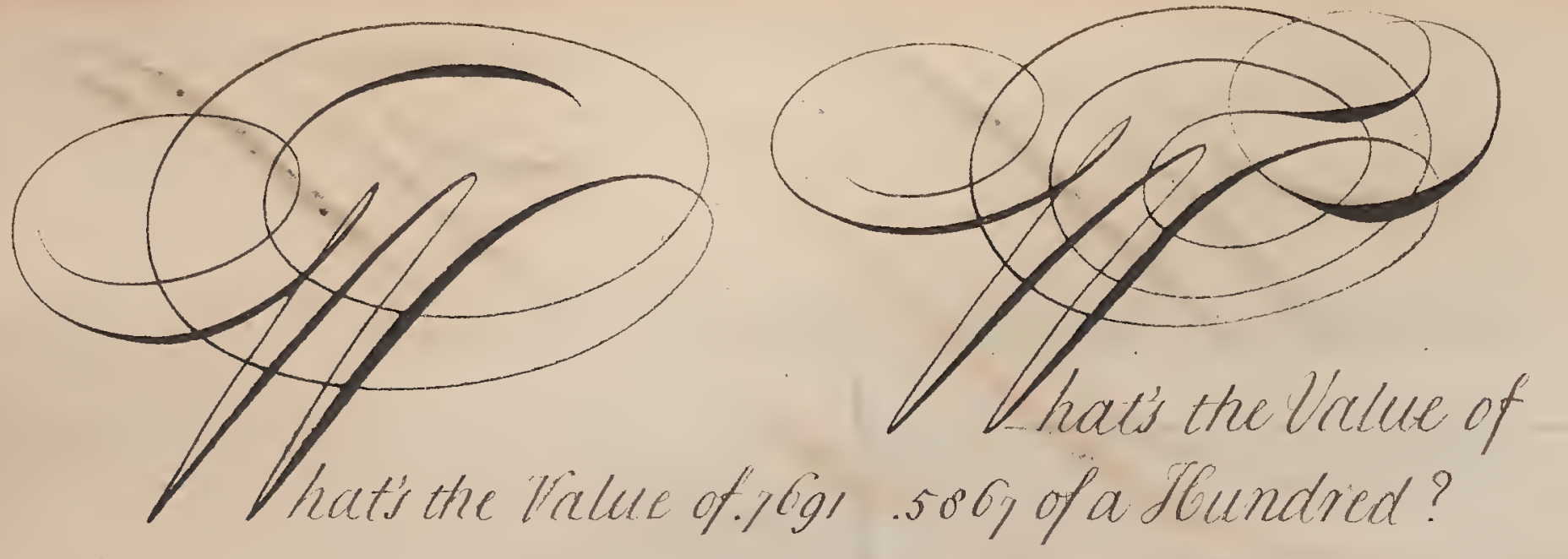
of a Son Averdupoise Weight?
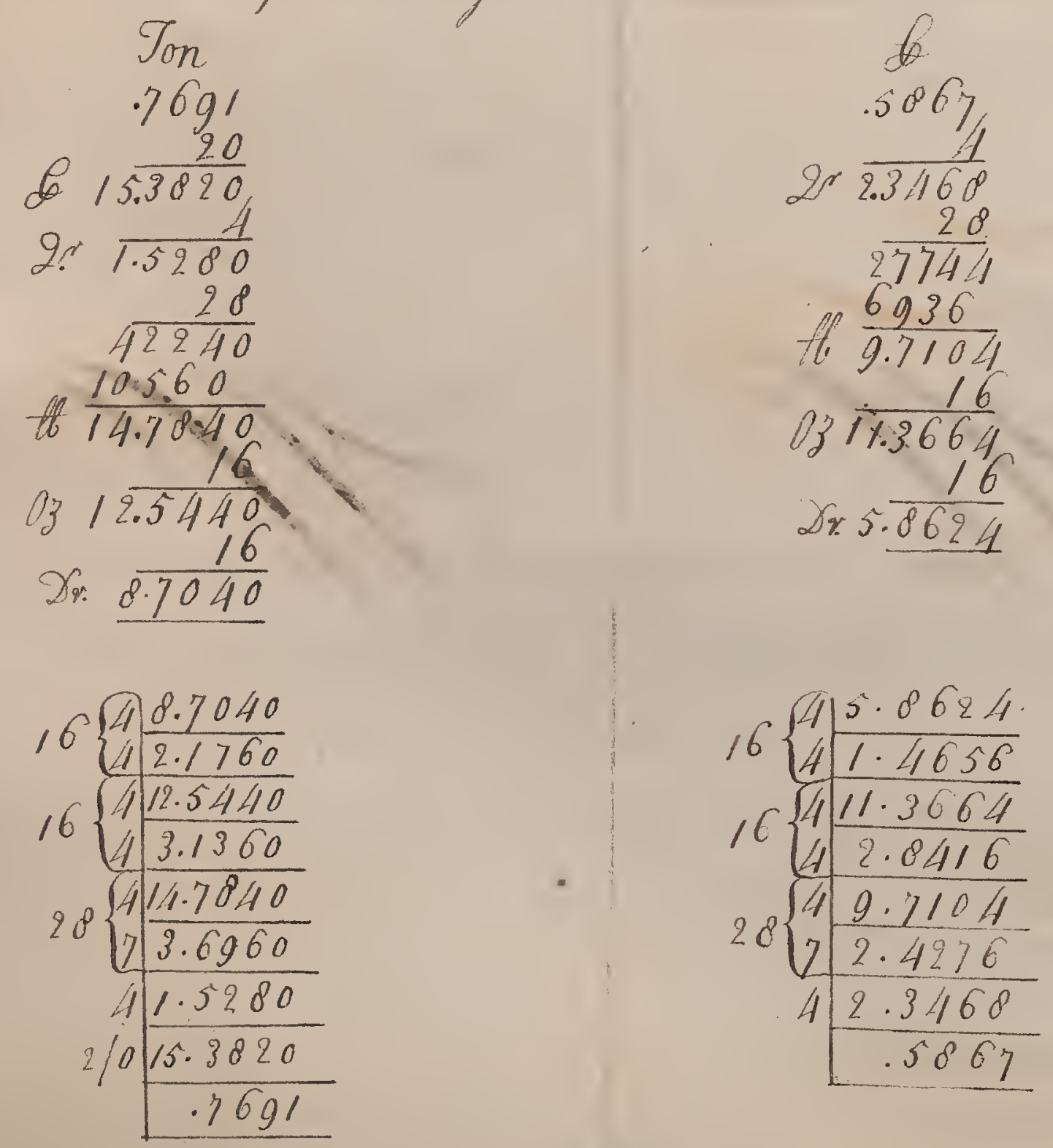
MSS 8388

RE HAH
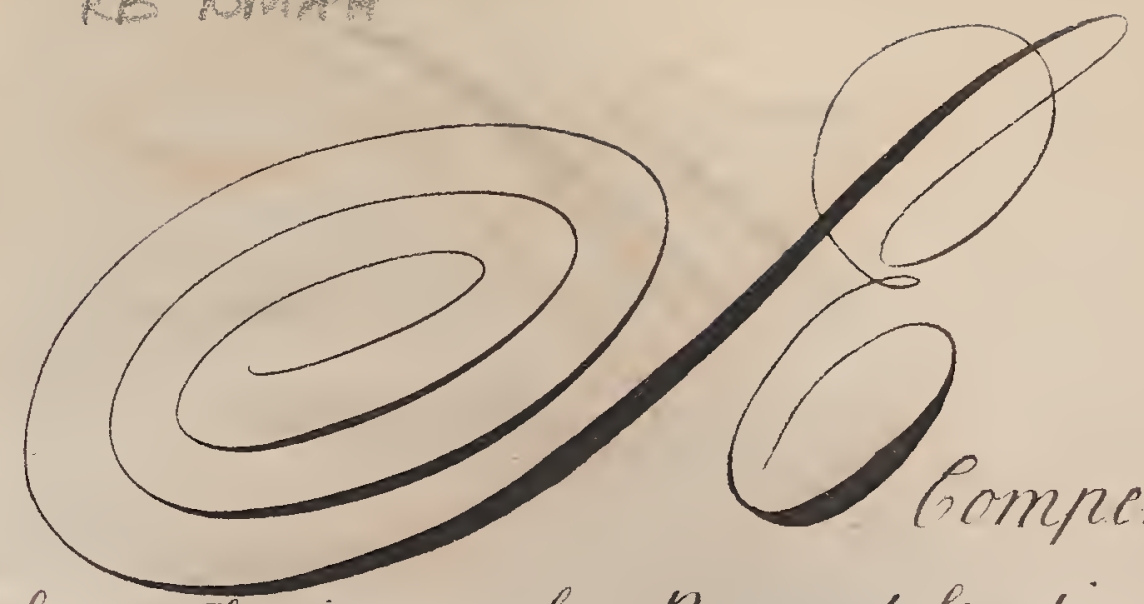

Compendious IVy to find the Vas of the Decimal of a found Sterling by Inspection.

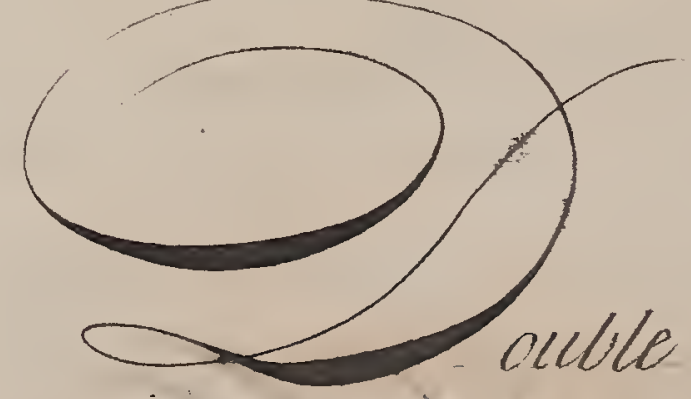

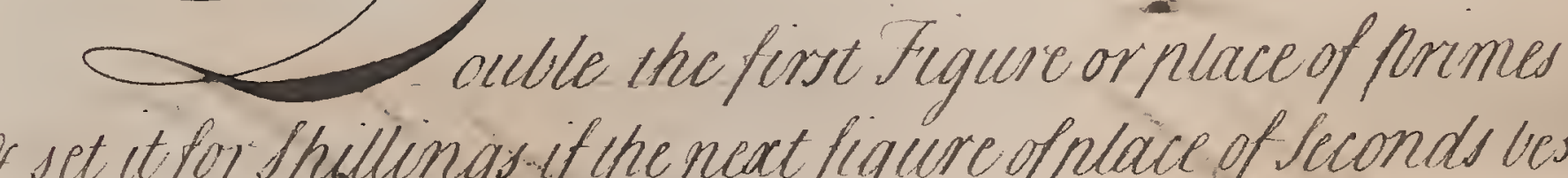
w se rt for shy ingsif the neat figure of place of Seconds les or more than s for ihs add it the former shillings: Then forevery Init in the 2." Place suppase'em as tens set before the sod place reckon these as far: but if they make above is abates or 2 if cloves s es these farthings when reduced to pence must lie added to the Shilling before found.
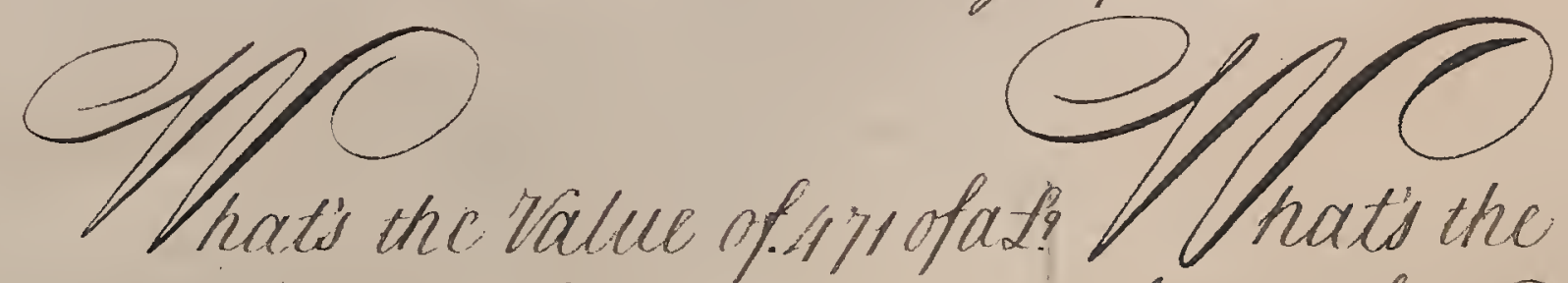

answering. of .270 of a Pound?

.15
.021
.471 proof answer is: a $\sigma^{d}$

$\frac{.25}{.020} \frac{.27 .0}{\text { pro of }}$ 


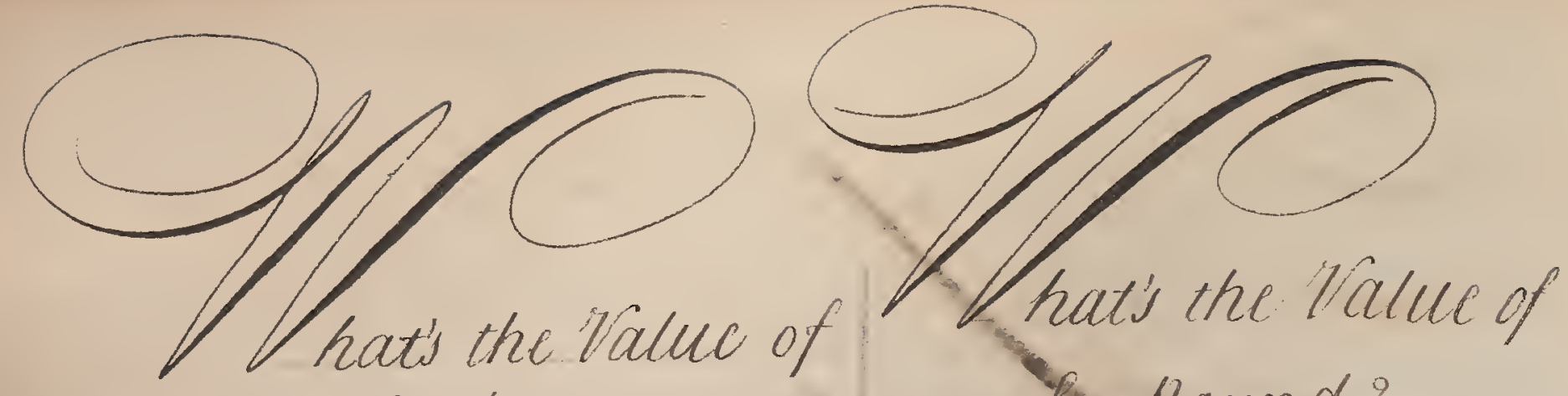

. 16 of a hound?

answer 18,38s

$.0 \% 1$ of relliund?

$\frac{.9}{.015}$ / hroof

Answer /: s

.0211 .021 Aroof

hatis the Value of.oog

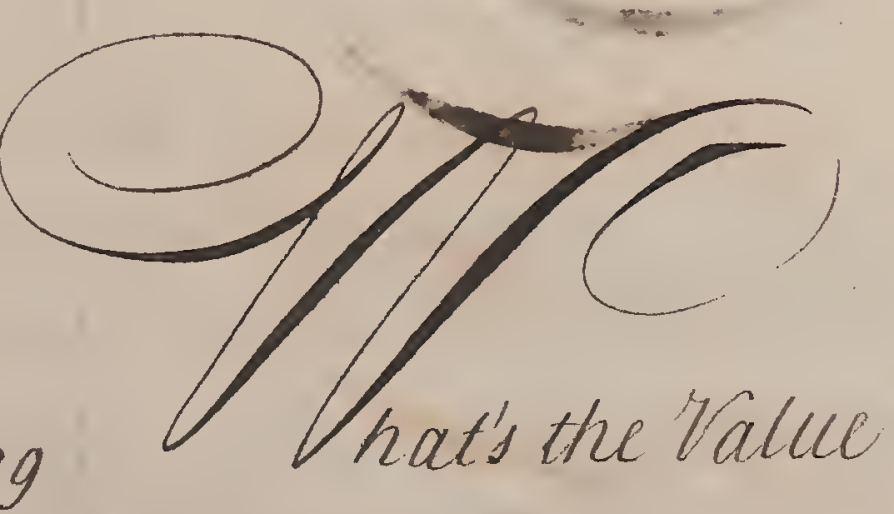

of af?

of oo es of a flound?

answer 2-4

Answer?

.009 froof

.000 froof 

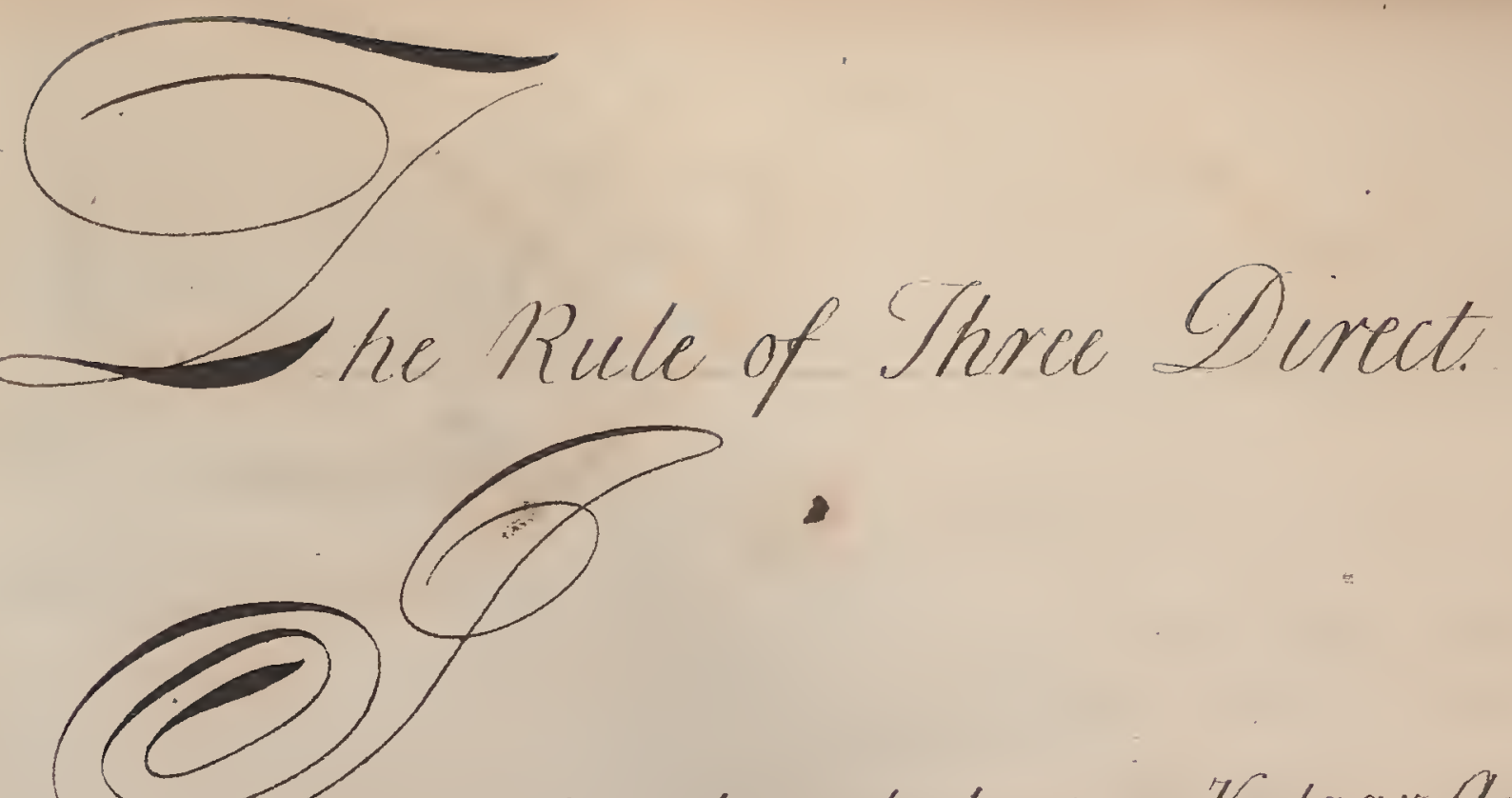

stated and worked as in Vulgar Aruhmelick only instead of preparing your Ilumbers by re. ducing them into their lowest Denominations You must Iring their Fractional Rarts into Decimals.

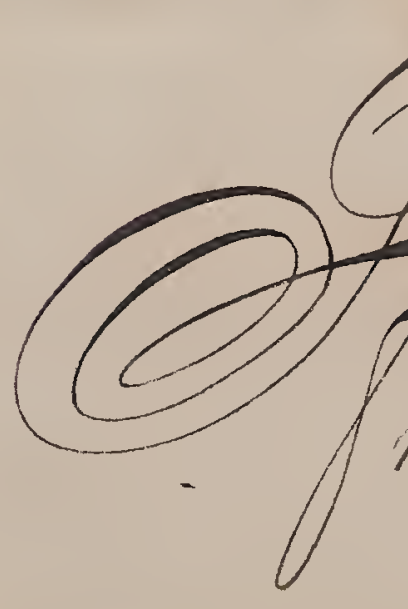

mill 32 y yarls cost at that Rete?

yos 1 s d ysu

$264: 3.16 .3: 324$.

$\frac{41 \frac{1.00}{26.25} \quad \frac{1213.00}{2 / 0 \frac{16.25}{3.0125}}}{\frac{32.25}{190625}}$

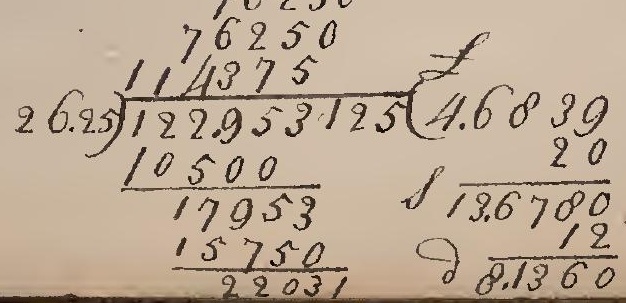

$2903 \%$

$\frac{21000}{10312}$ $\frac{7875}{24.375}$

$\frac{23625}{750}$ 


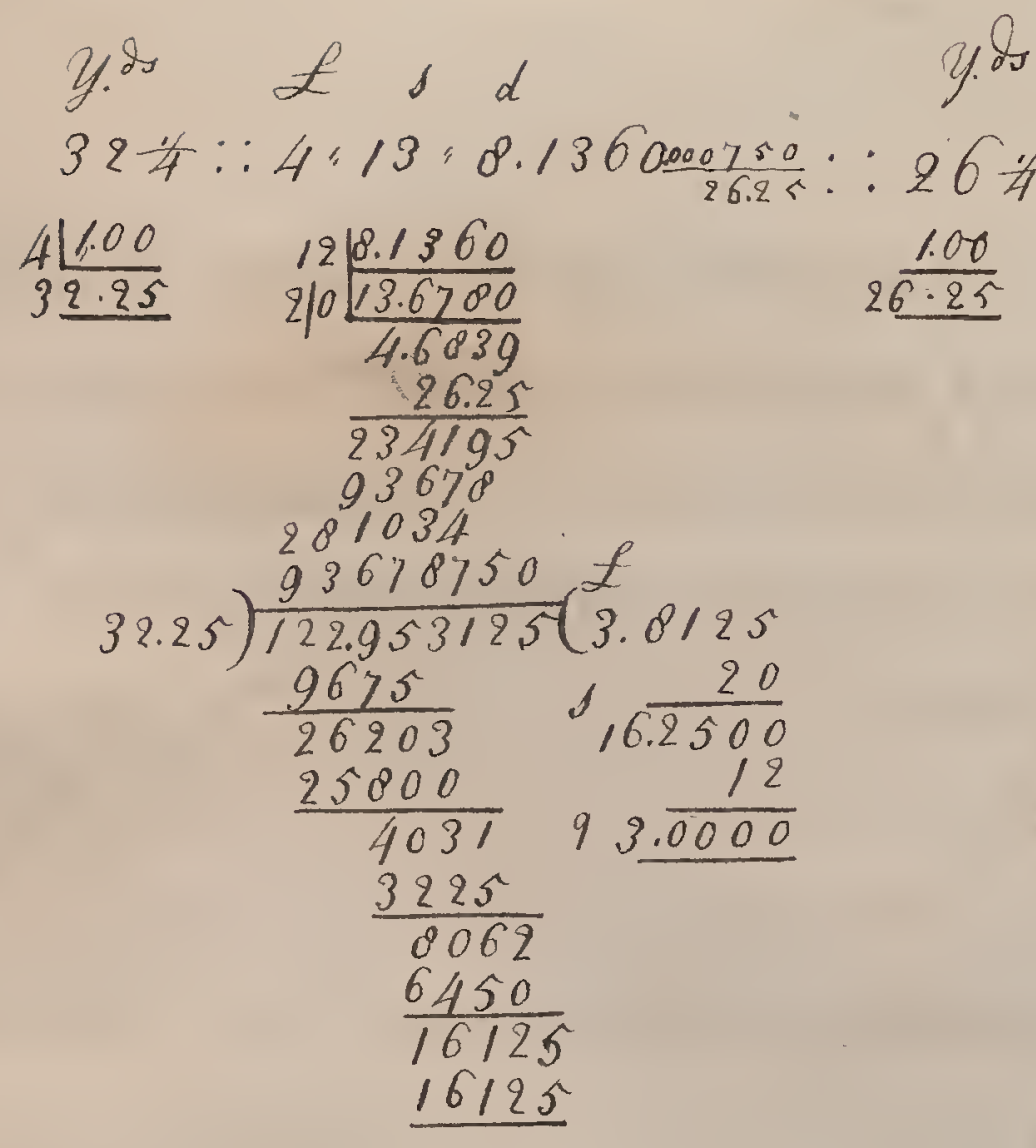




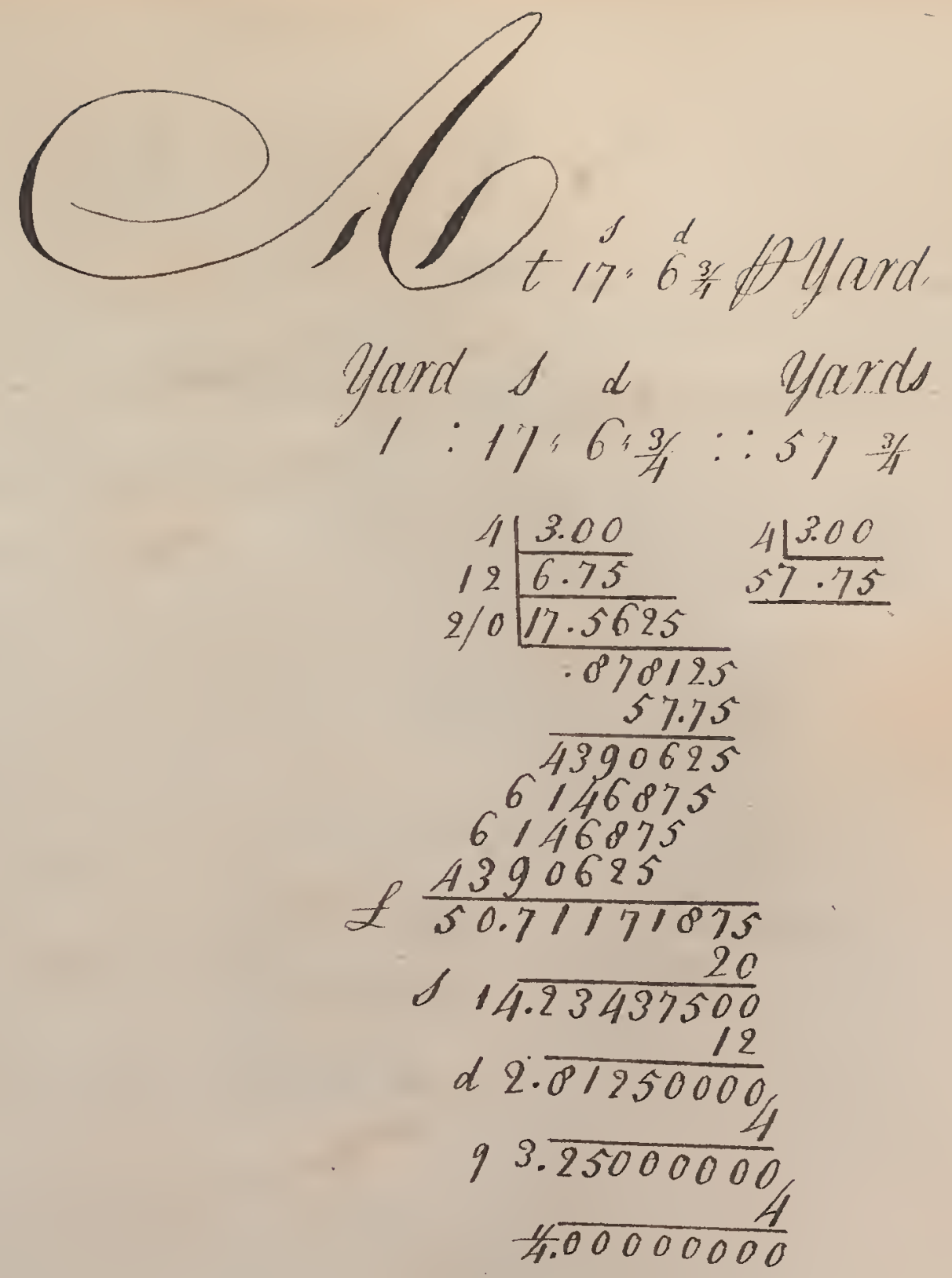




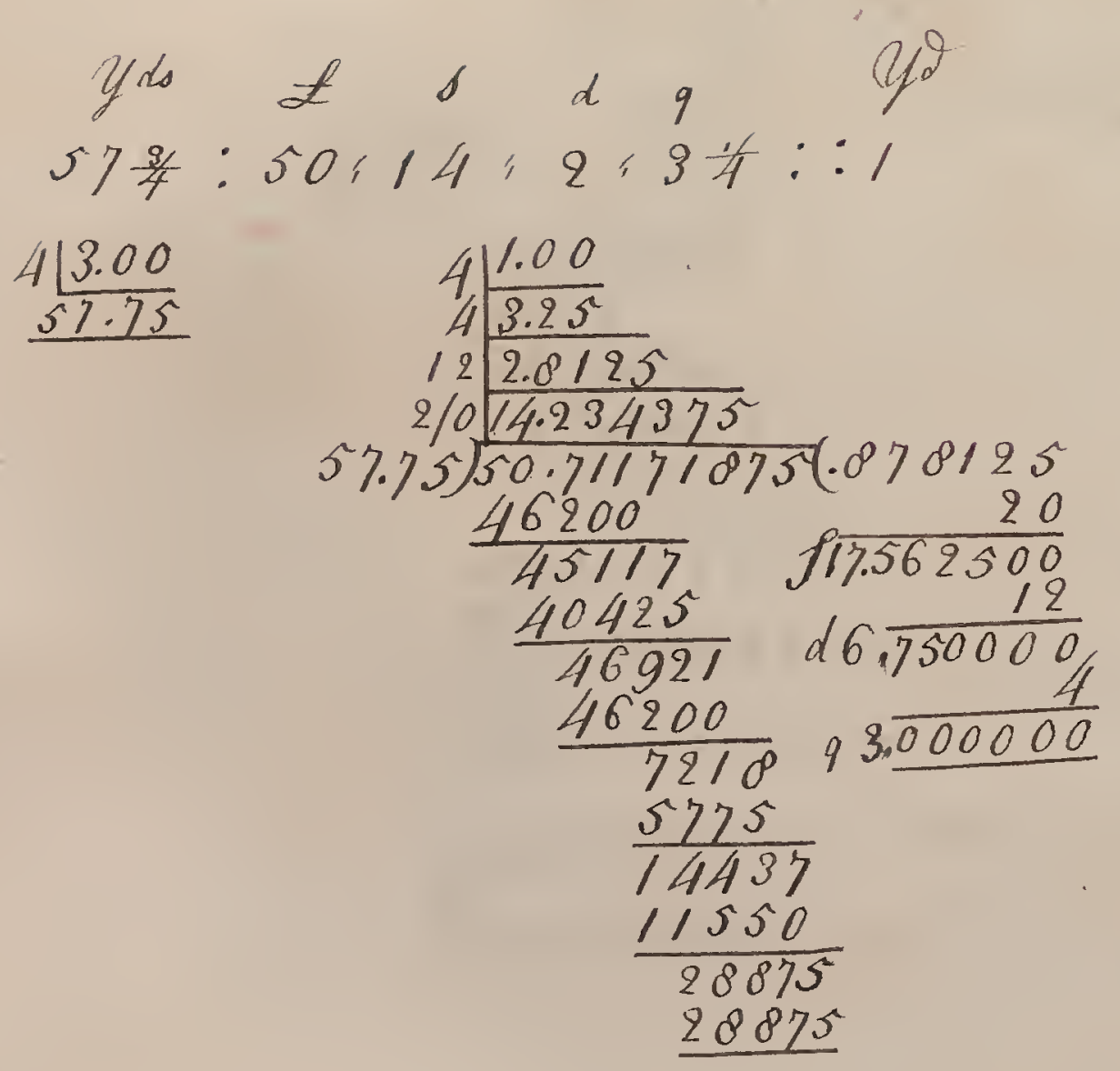




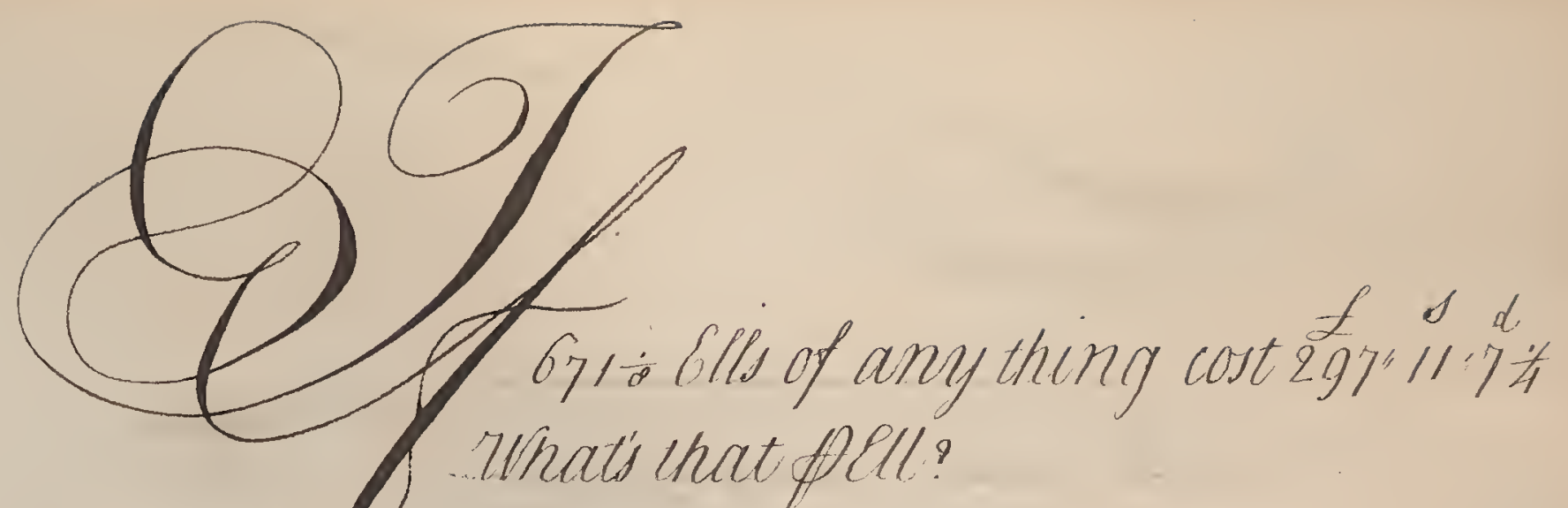

Gyis blis of any

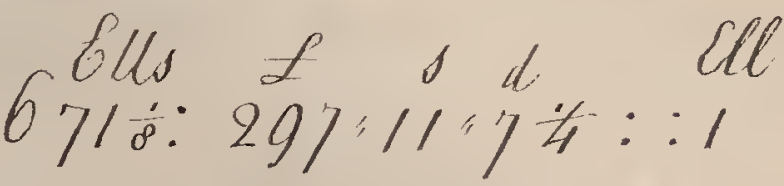

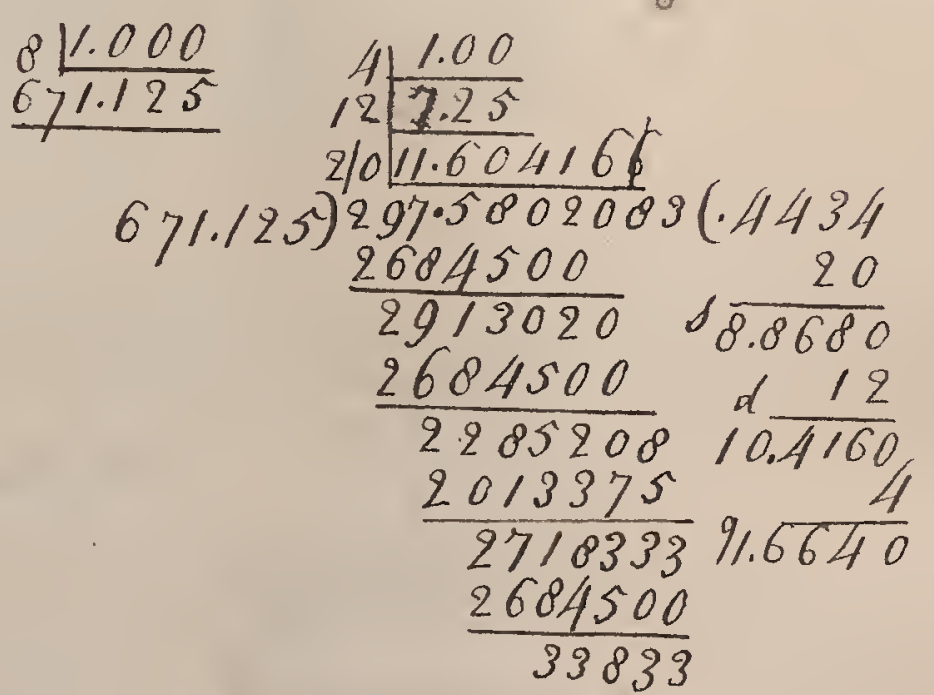


oll. s d 9

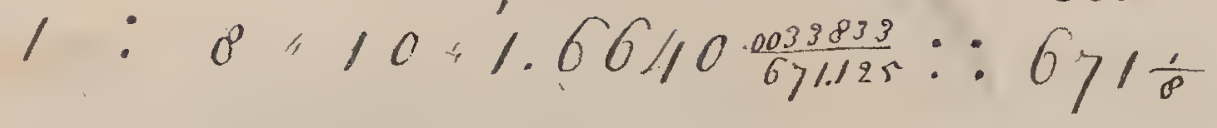

$$
\begin{aligned}
& \begin{array}{r}
4 \frac{1.6640}{10.4160} \\
2 / 0 \frac{8.0600}{.4434} \\
\frac{6.4125}{20}
\end{array} \\
& \frac{671.125}{22170} \\
& 0860^{\circ} \\
& 4434 \\
& 4434 \\
& \mathcal{L} \frac{36038}{266433833} \\
& 111.6041660 \\
& \text { d) } \frac{12}{2499920} \\
& 9989680
\end{aligned}
$$

$\frac{81.000}{671.125}$

Ells 

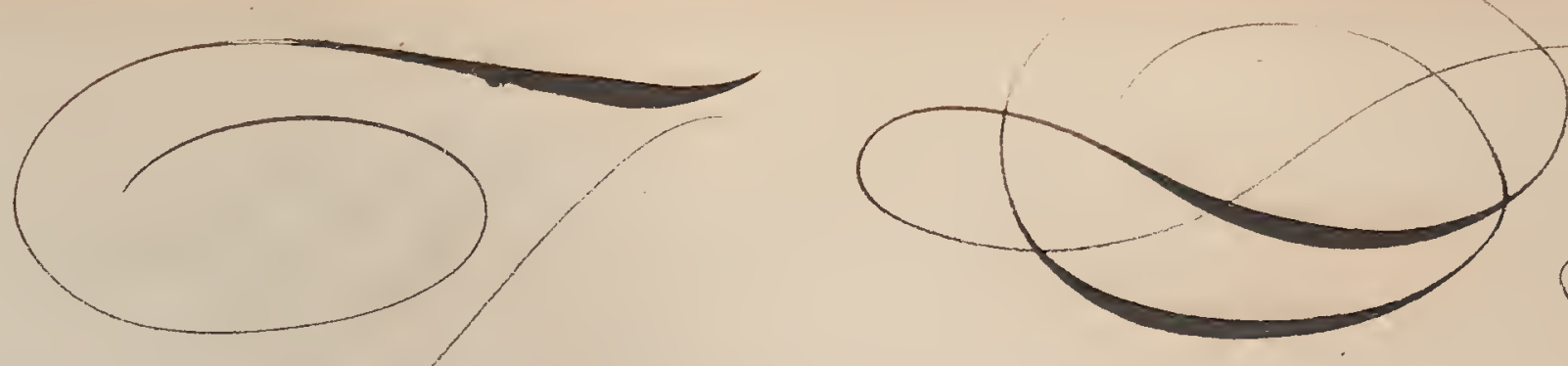

he Indirrect Rule of Storee.
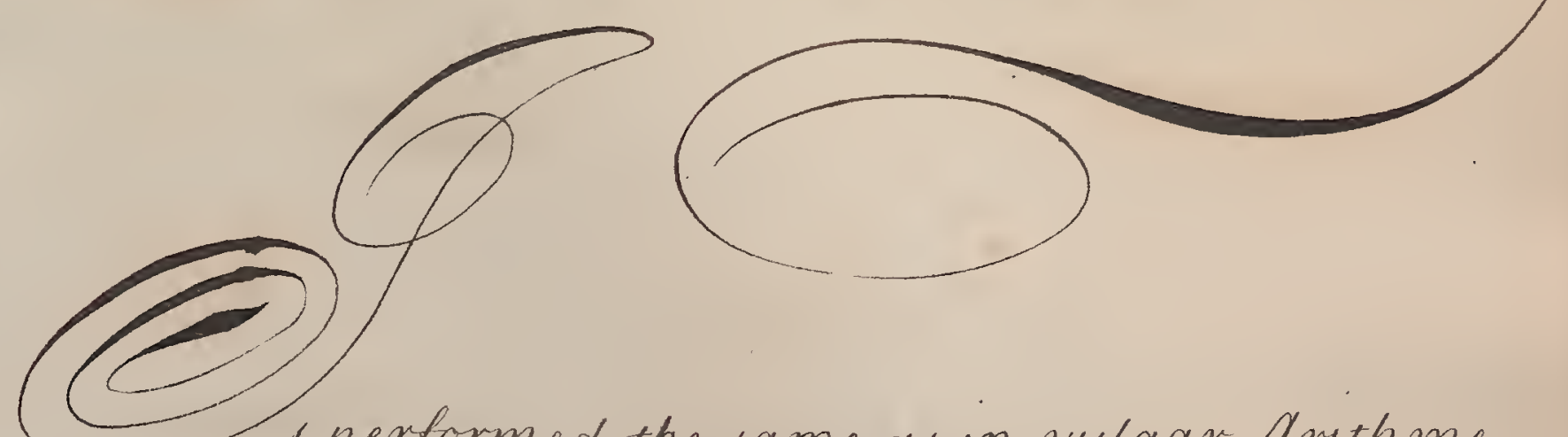

- tick after the Nlumbers are prepared nstaught in Decimals.

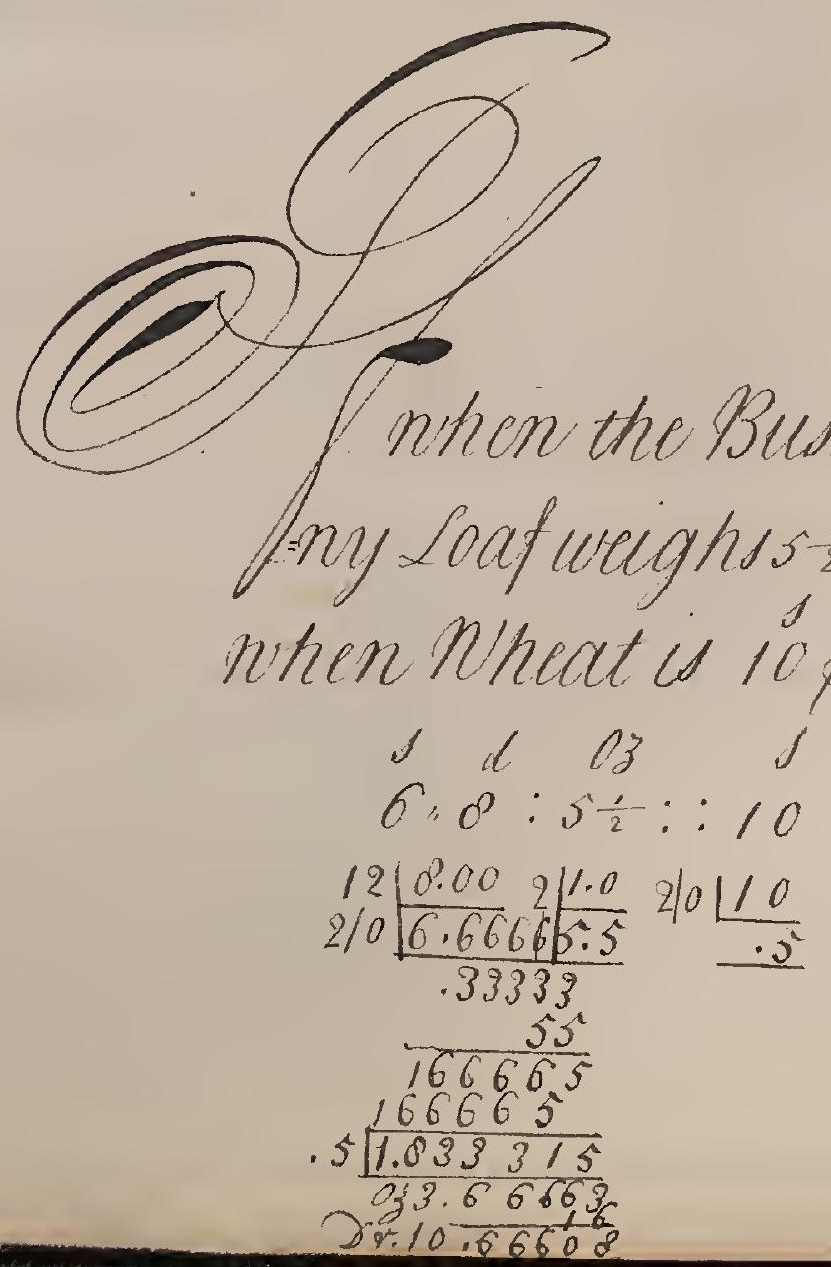




$$
f_{10}: 3: 10.66608:: 6: 8
$$

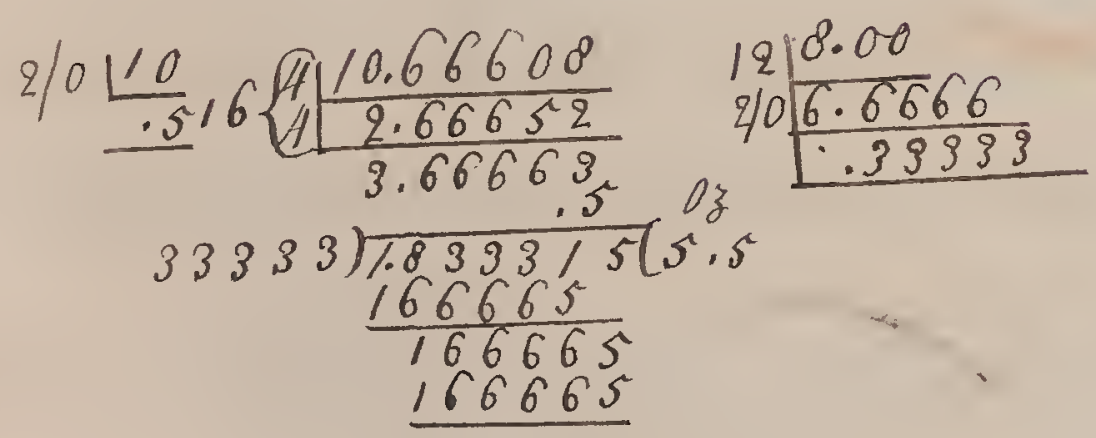




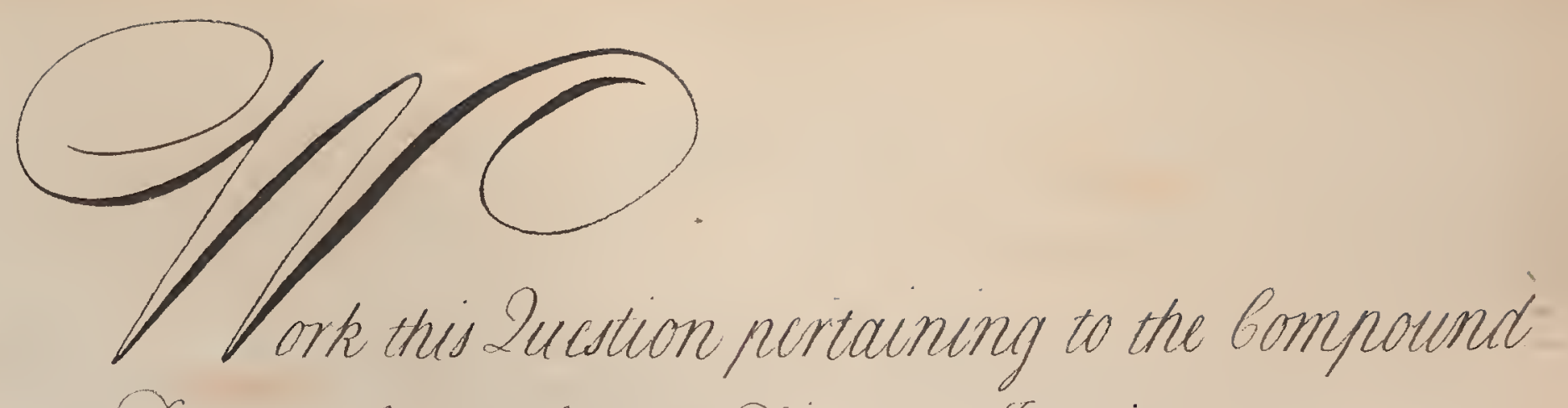
or Doubte Rule of three Direct\& Indirect.

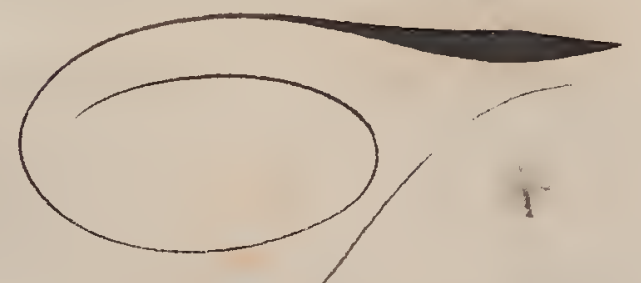

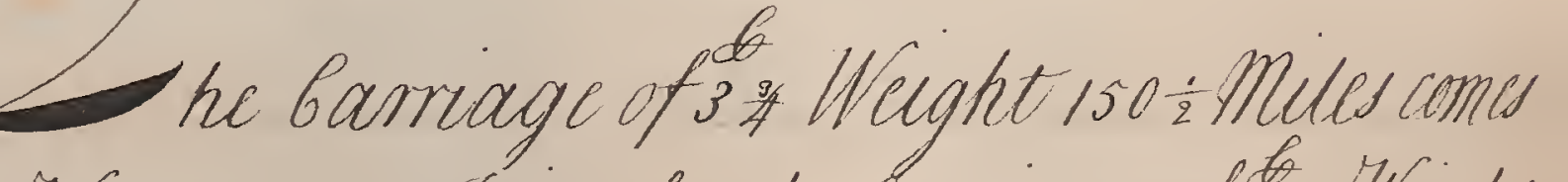

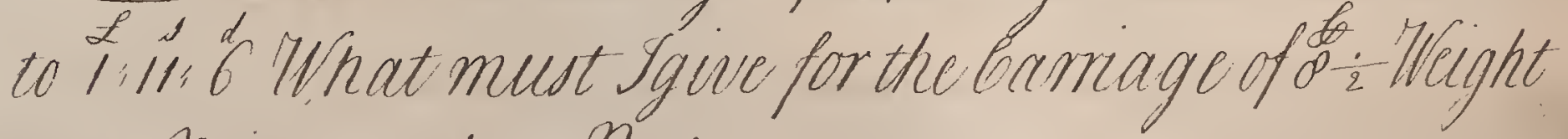
s.2: Miles at that Rate?

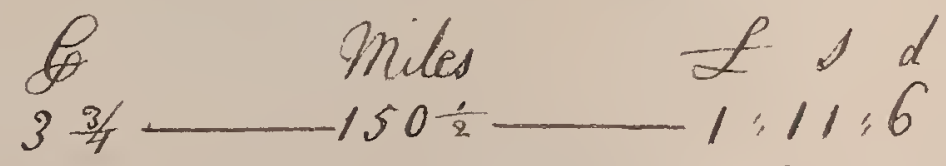

$$
\begin{aligned}
& 8 \frac{1}{2}-50 \frac{1}{2}=* \\
& 4 \frac{3.00}{3.75} \quad \frac{211.0}{250.5} \quad \frac{12}{210} \frac{6.0}{1.5} \\
& 2 \frac{1.0}{8.55} \quad \frac{1.0}{32.5} \quad \frac{0.5}{7870^{\circ}} \\
& \frac{12600}{133875} \\
& \frac{150.5}{3.75}
\end{aligned}
$$

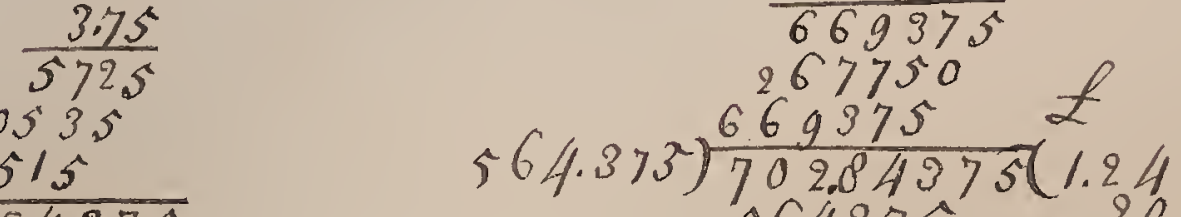

$$
\begin{aligned}
& \frac{4515}{564.375} \\
& \frac{564375}{138460^{7}} \& \frac{20}{4.80} \\
& \frac{1128750}{2559375} \cdot \frac{12}{604} \\
& \frac{22575009.240}{301875}
\end{aligned}
$$




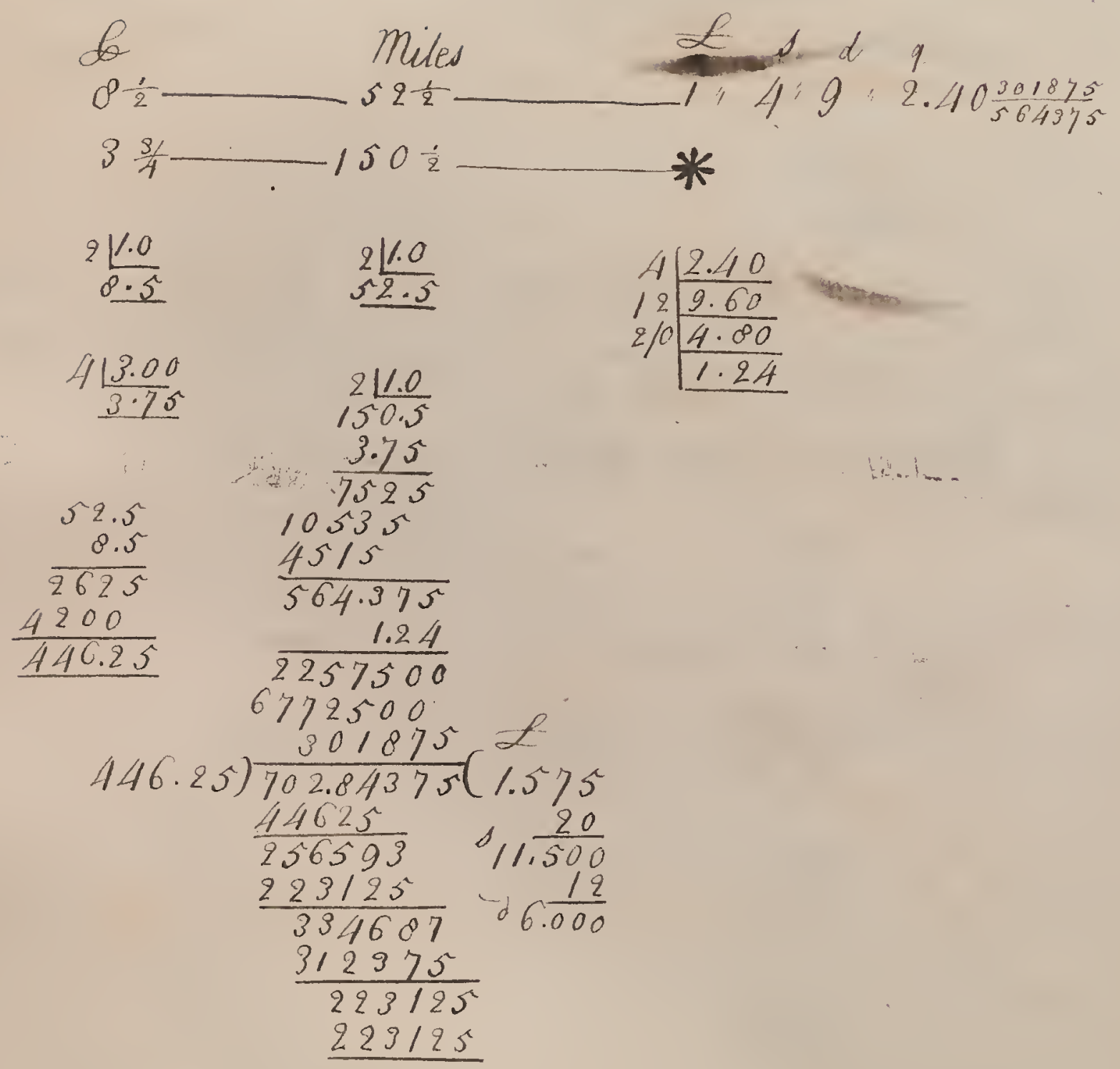




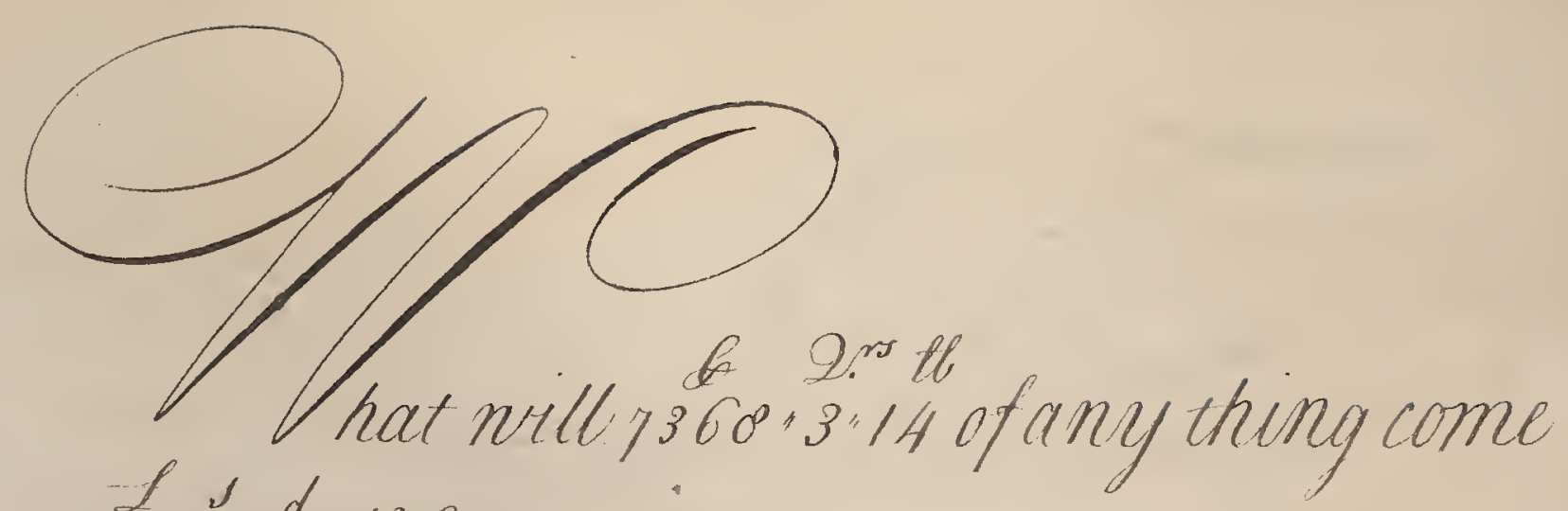
to at 1"12" 6 tiff?

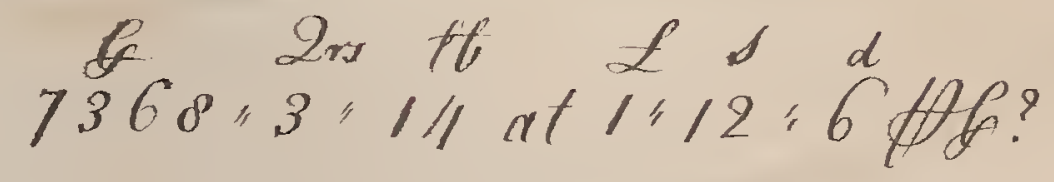

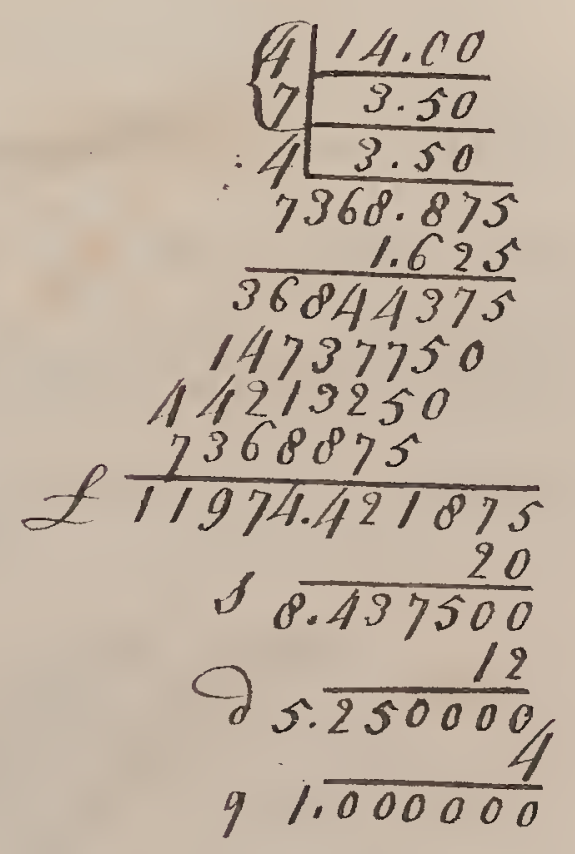

$$
12 \frac{\frac{6.00}{12.50}}{1.625}
$$





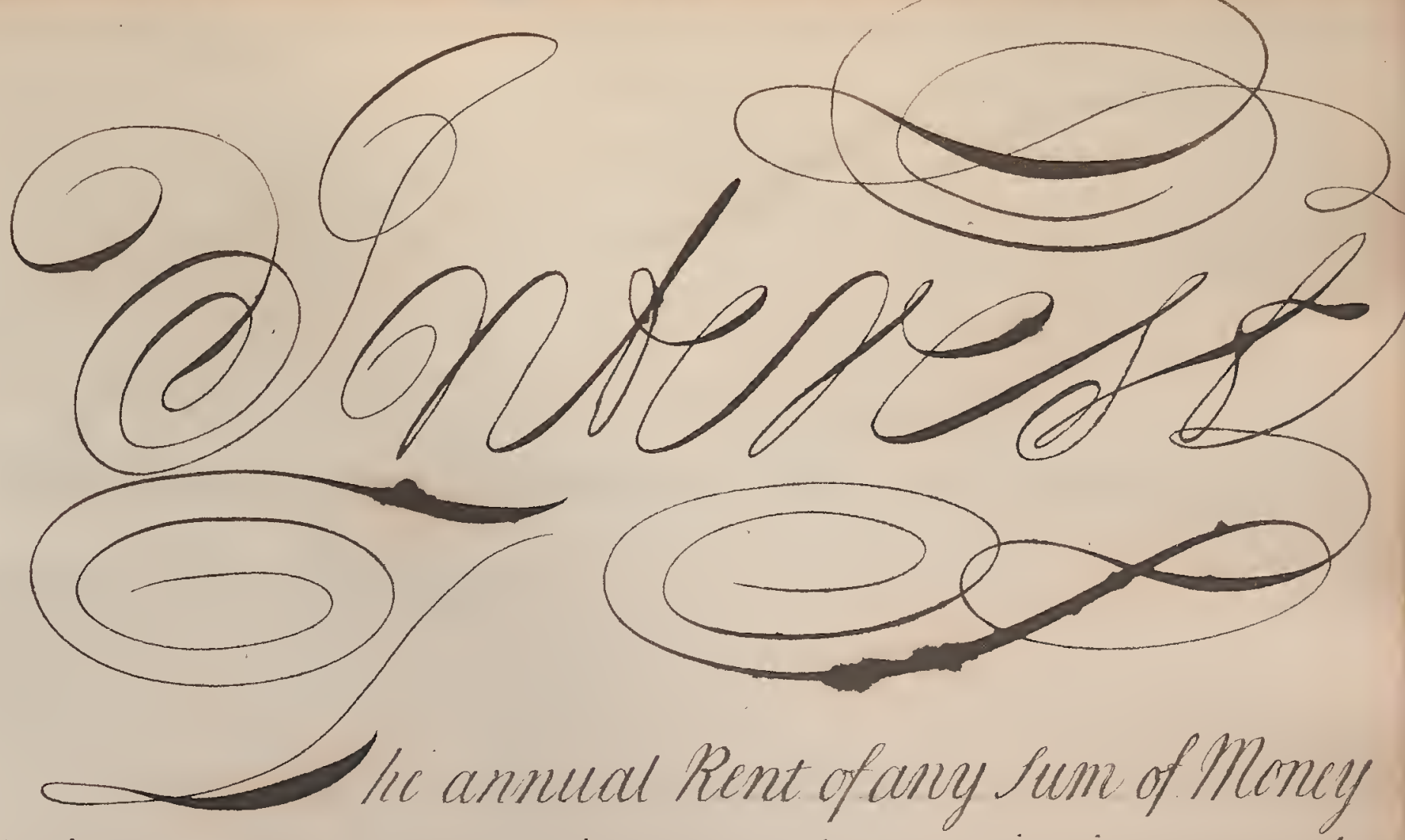
is found by multiplying the given prinipal by the Interest of , pound for a Your, n'thich is found by dividing the Rate of Sutercst floo.

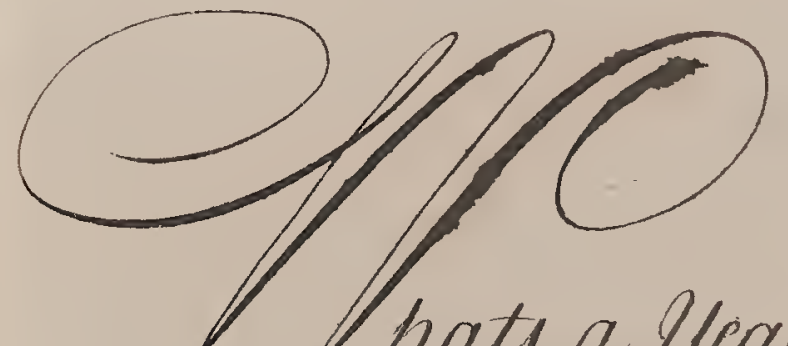
hats a years' Snterest of $102=14: 6$ at 4 focent:pannum? 702:11: 'd

$$
\begin{array}{r}
12 \frac{6.00}{\frac{64.50}{782.725}} \\
\mathcal{L} \frac{.04}{31.30900} \\
\& 6 \frac{20}{1.18000} \\
d \frac{12}{2.16000} \\
\frac{.64000}{164}
\end{array}
$$



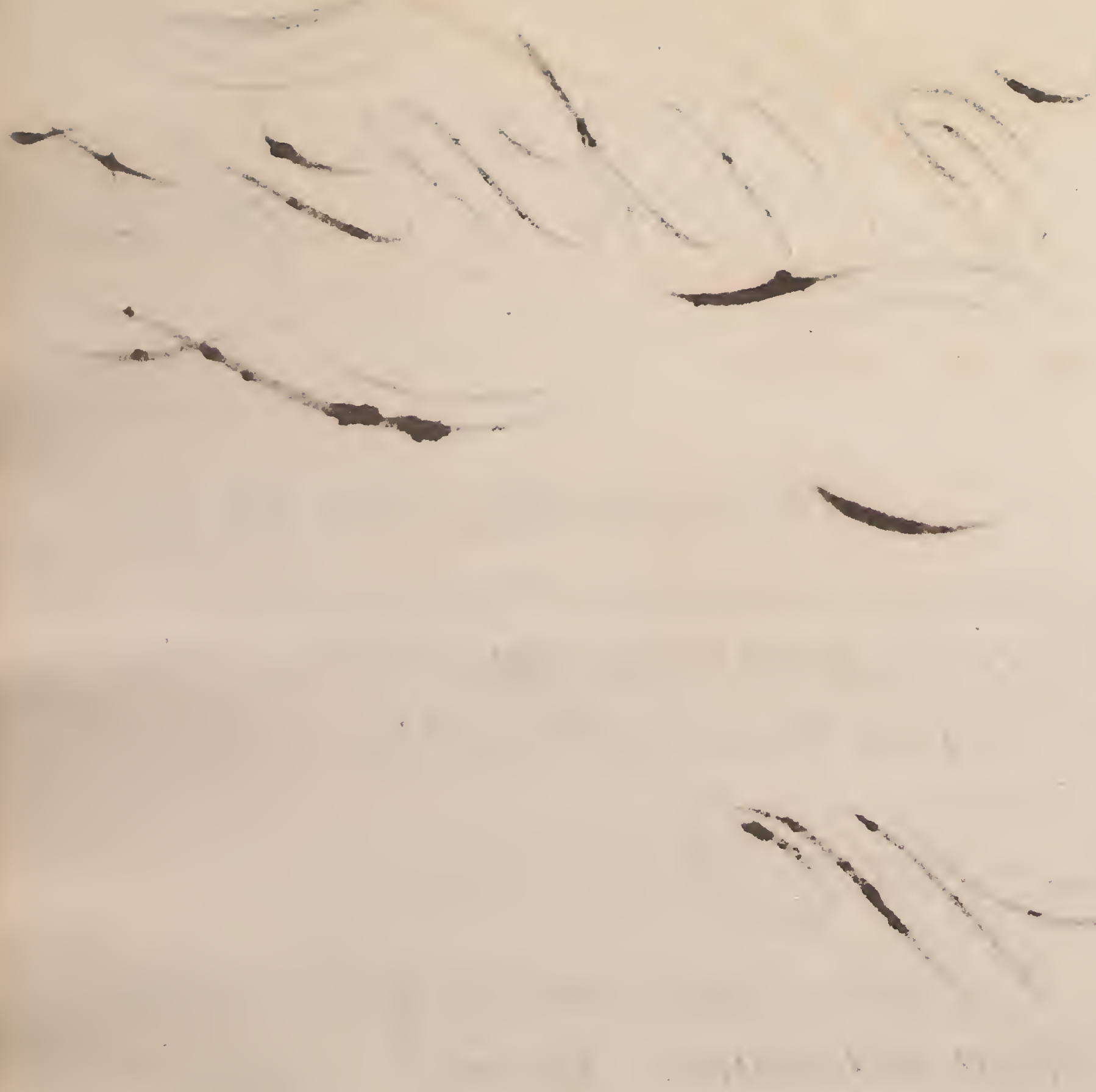


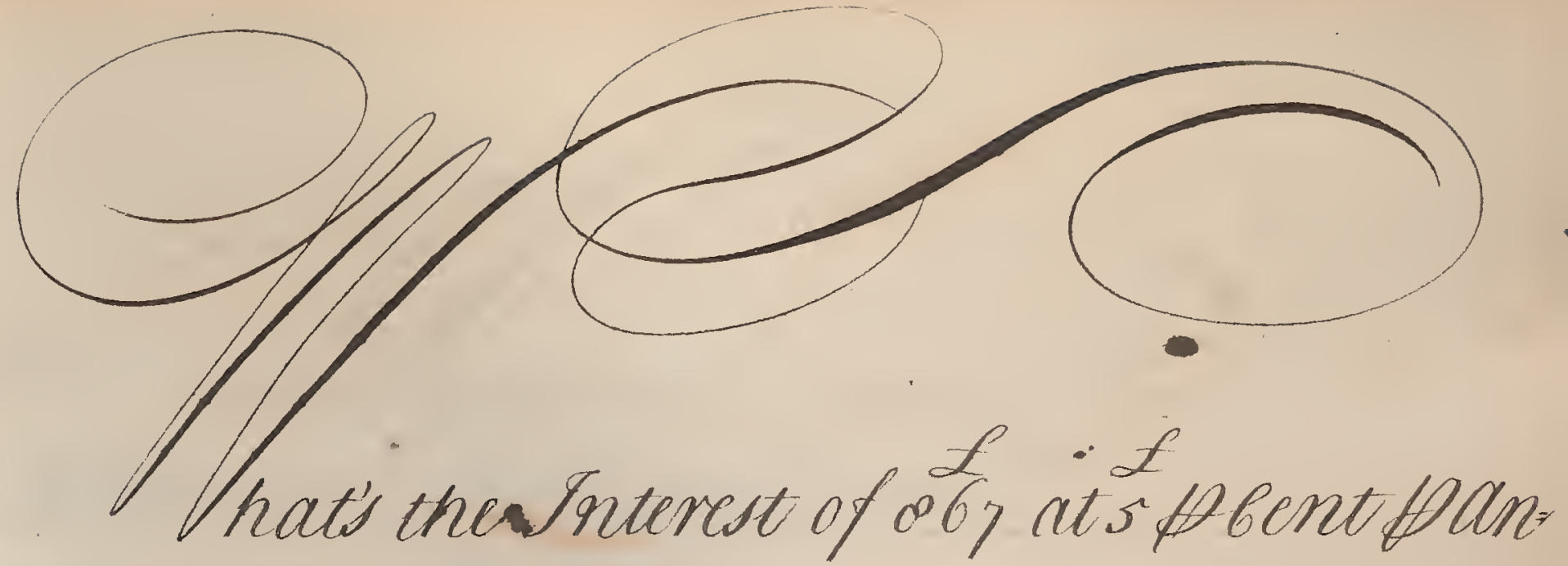

-num, for 2 Years?

$$
\begin{aligned}
& \mathcal{L} \\
& 867 \\
& \frac{.05}{43.35} \\
& 57.000
\end{aligned}
$$$$
\begin{array}{r}
7 \\
13: 5 \\
\frac{2}{86.14} \text { answer }
\end{array}
$$ 



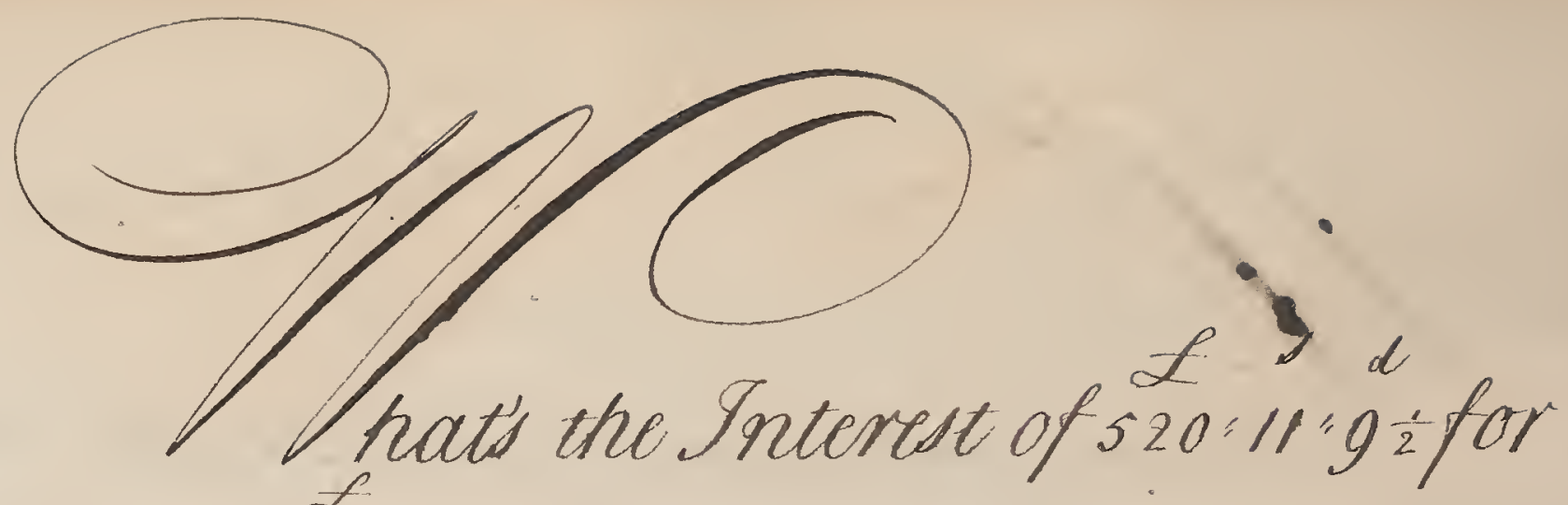

$5 \frac{1}{2} y$ ears at of pbent pannum?

s. so" $11 " d+$

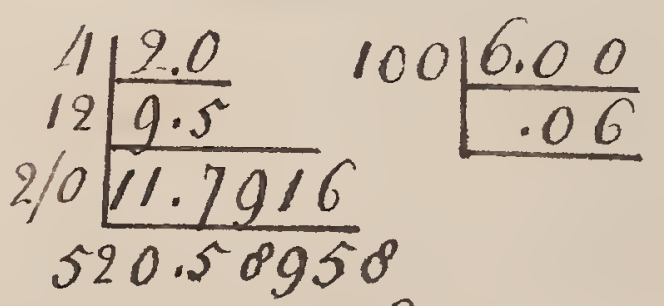

$$
\begin{aligned}
& \frac{.06}{31.2359740^{\circ}} \\
& 14.7074960 \\
& \text { D } 8 . \overline{4899520} \\
& \text { q } 1.9598080 \\
& \begin{array}{l}
\mathcal{L}, 9,9 \\
31.4: 0: 1.959008 \\
156: 3: 6: 1.799040 \\
15: 12: 4: 0.979904 \\
\hline 111: 15: 10: 2.770944 \\
\hline
\end{array}
\end{aligned}
$$




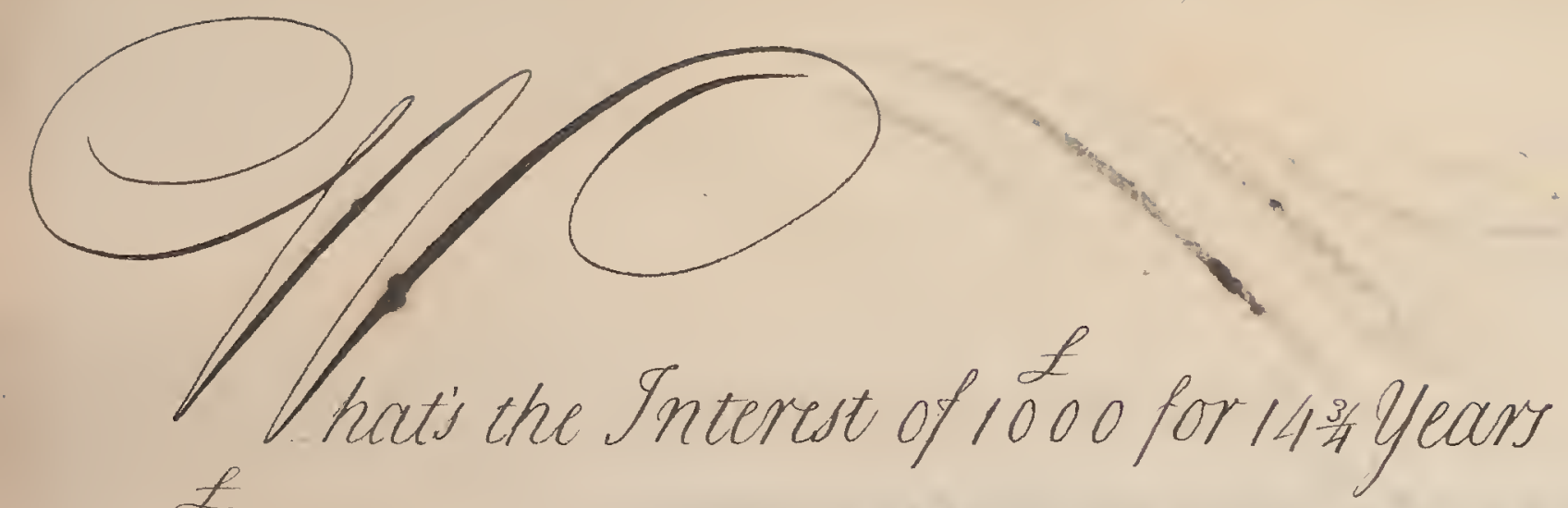
at $\frac{f}{6}$ plent: fannum?

$$
\begin{aligned}
& \frac{\mathscr{L}}{1000} \\
& \frac{.06}{60.00} \\
& \\
& \mathscr{L} \frac{14.75}{80500}
\end{aligned}
$$$$
100 \frac{6.00}{1.06}
$$ 


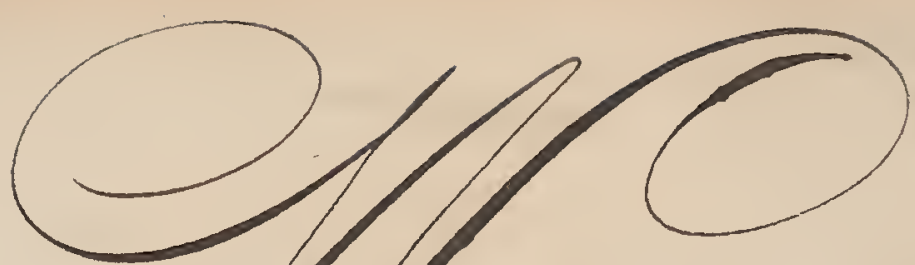

120 Darys at th pent pannum?

$$
\begin{array}{r}
.00016438356 \\
001621 \\
00032076356 \\
00098630136 \\
\hline 0.10208219076 \\
f_{12.24986289120}^{120} \\
14.99725782400 \\
d 11.96709388800 \\
19.86037555200
\end{array}
$$




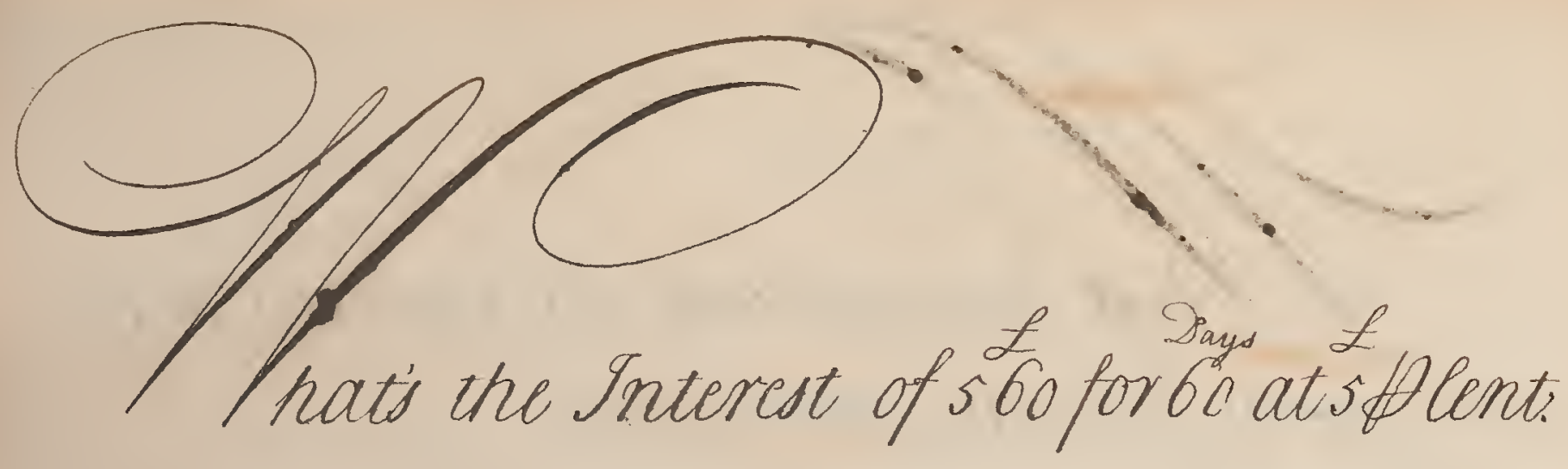

toannum?

$$
\begin{array}{r}
.00013698630 \\
60 \\
0.00821917800 \\
0049315068000 \\
\frac{4709589000}{4.60273968000} \\
d 19.05479360000 \\
d 0.65752320000 \\
q 2.63009280000
\end{array}
$$




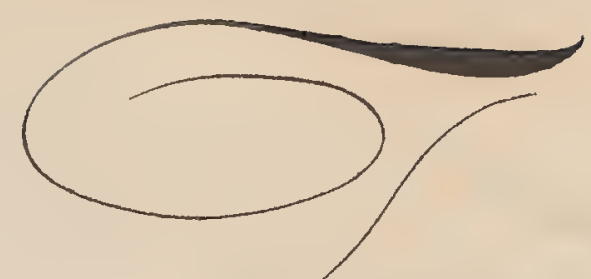

the Interest of $1_{\text {for }}$, Day is thus found the given Rate or Interest of 100 for a Year being di= vided p100 quotes the Interest of I for a Ylear, which again divided p36s gives the required Inuerse of if or iDay: which when Mulliplyed into both ithe Rumber of

Danys the principal fum produces the Sruterset for timere guired.

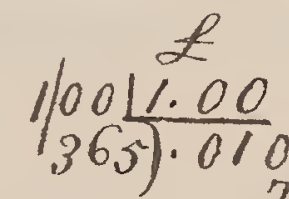

$$
\begin{aligned}
& 730 \\
& \frac{7300}{2700} \\
& \frac{2555}{1450} \\
& \frac{1095}{3550} \\
& \frac{3205}{9650} \\
& \frac{9555}{950} \\
& \frac{790}{9200} \\
& \frac{2100}{2190}
\end{aligned}
$$

The Interest 12 for 1 Cay.

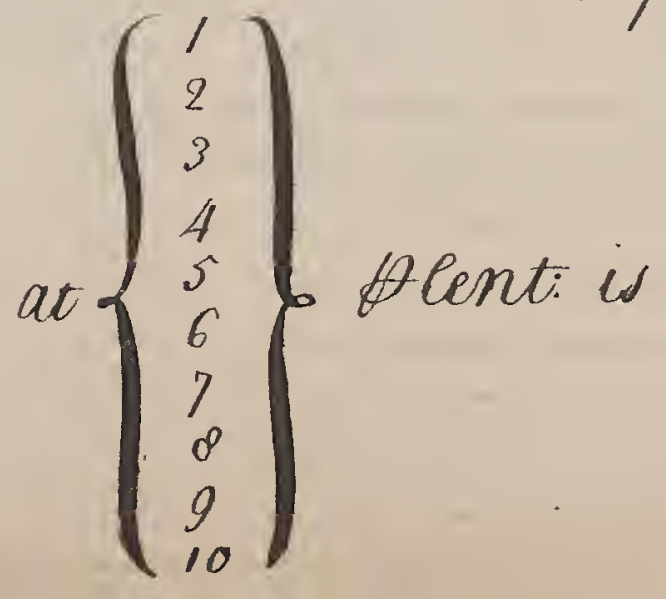

$$
\left\{\begin{array}{l}
.00002739726 \\
.00005479452 \\
.00000219170 \\
.00010950904 \\
.00013698630 \\
.00016438356 \\
.00019177002 \\
.00021917808 \\
.00024657594 \\
.00027997260
\end{array}\right.
$$






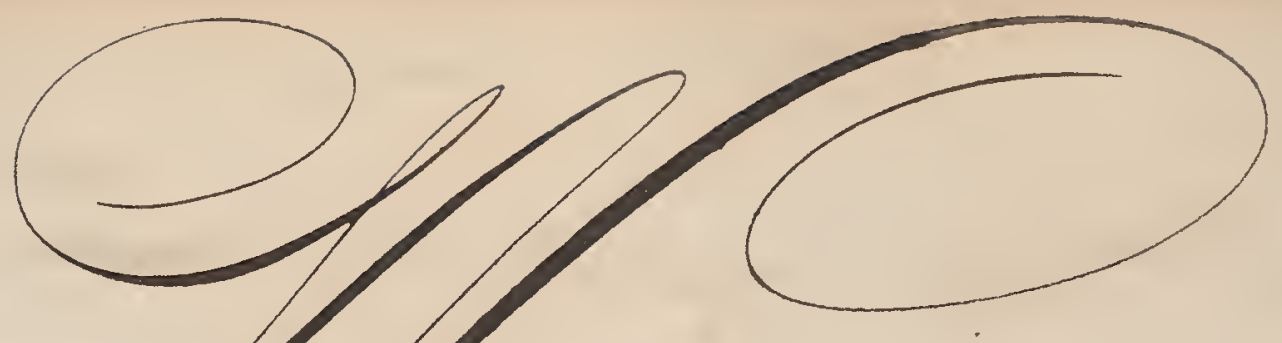

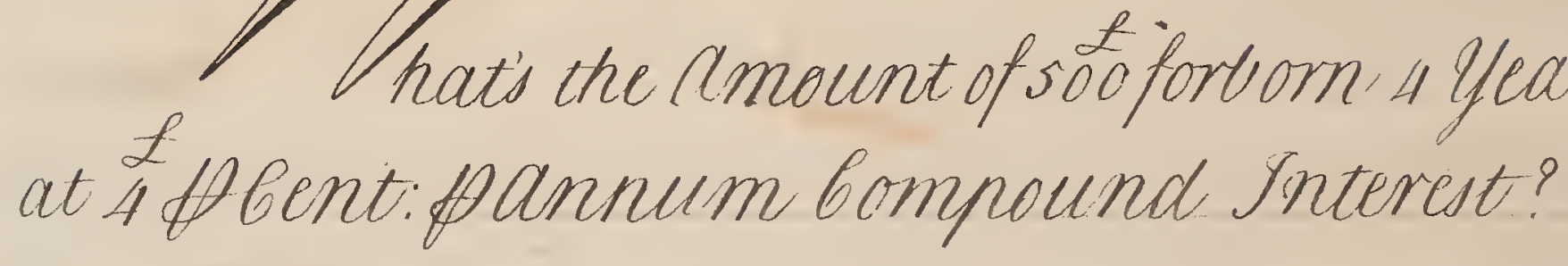

hat's the Imount of soofortorn a lears
pbent:pannum bompound Snterest?

$$
\begin{aligned}
& 1.04 \\
& \frac{1.04}{4.16} \\
& \frac{1040}{1.0816} \\
& \frac{1.04}{43264} \\
& \frac{108160}{1.124864} \\
& \mathcal{L}_{584.92928000} \\
& 818.58560 \\
& \text { d } 6.02720 \\
& . \frac{4}{10880}
\end{aligned}
$$




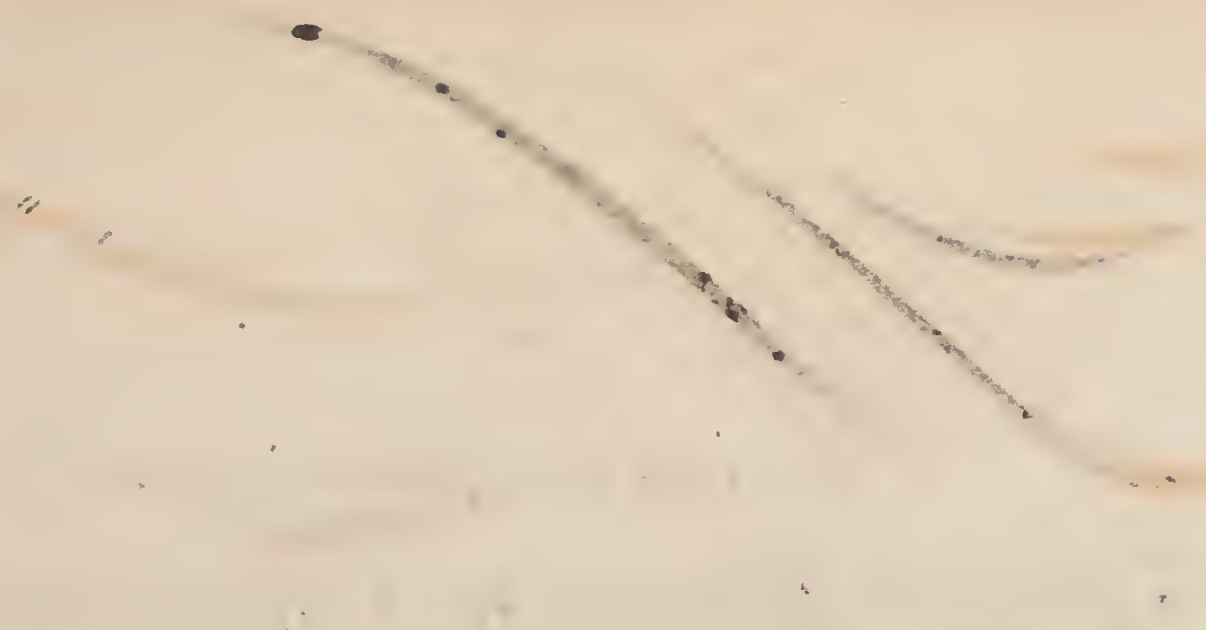




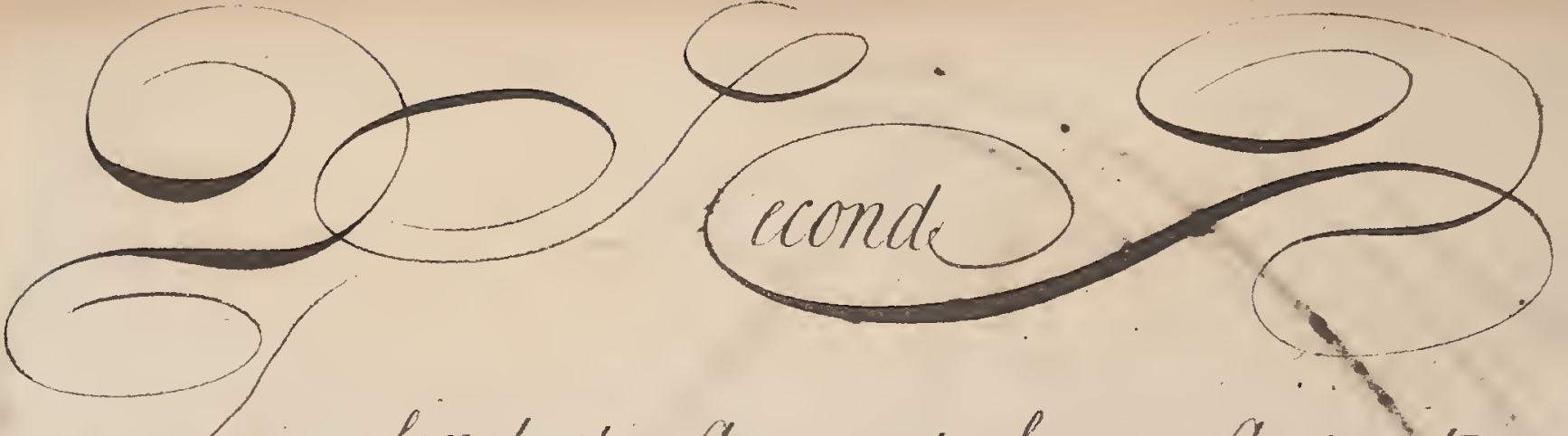

- find the Imount of any Annuisty ox Year. ty flension, fortion any Number of Yeairs vhatsoe= ver at any Rate of bompound. Interest.

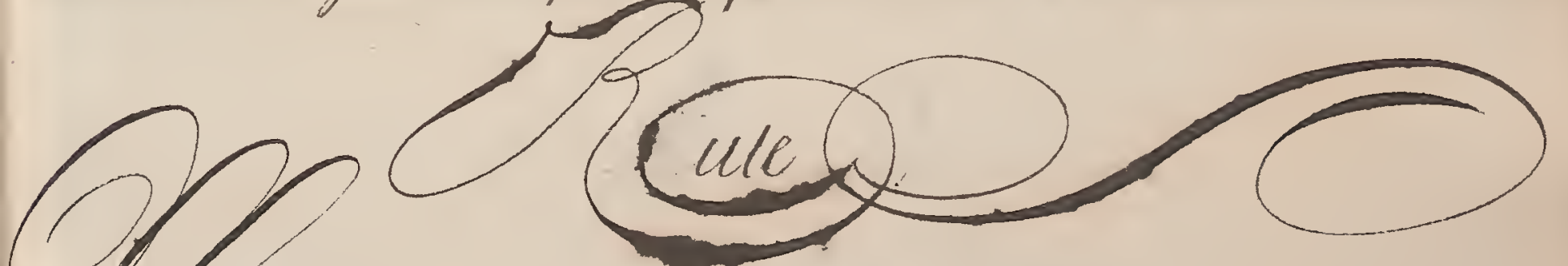

I Sultiply the first Yearly Pryment oy the Rate \& to the flroduct add the Sccond Yearly Rayment the Sum is the Amoune in 2 Yeans nhich multiphyed again by the Rate, the product rvith the Addition of the third yearly hayment is the Mmount for 3 Years. \&C. 


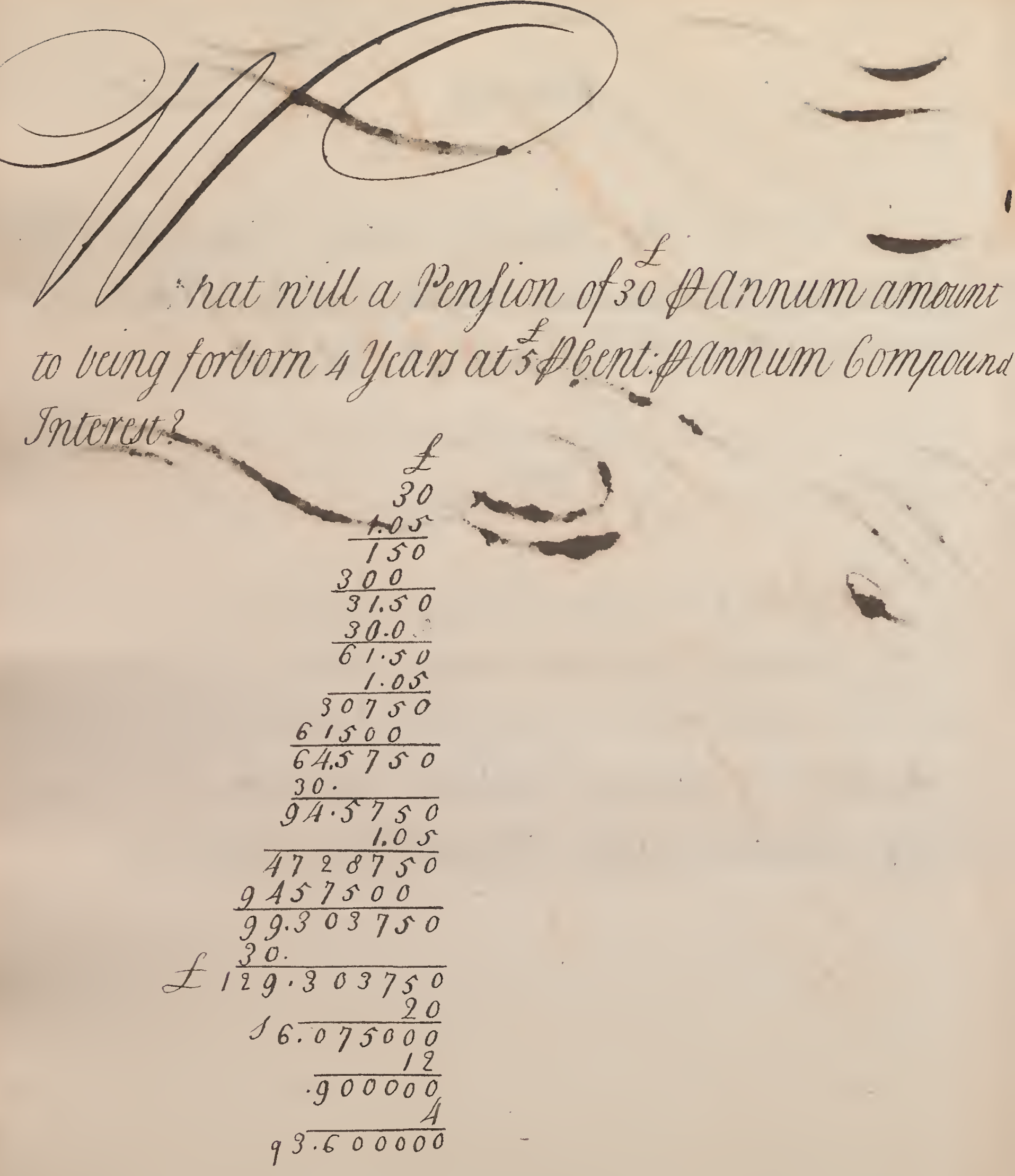




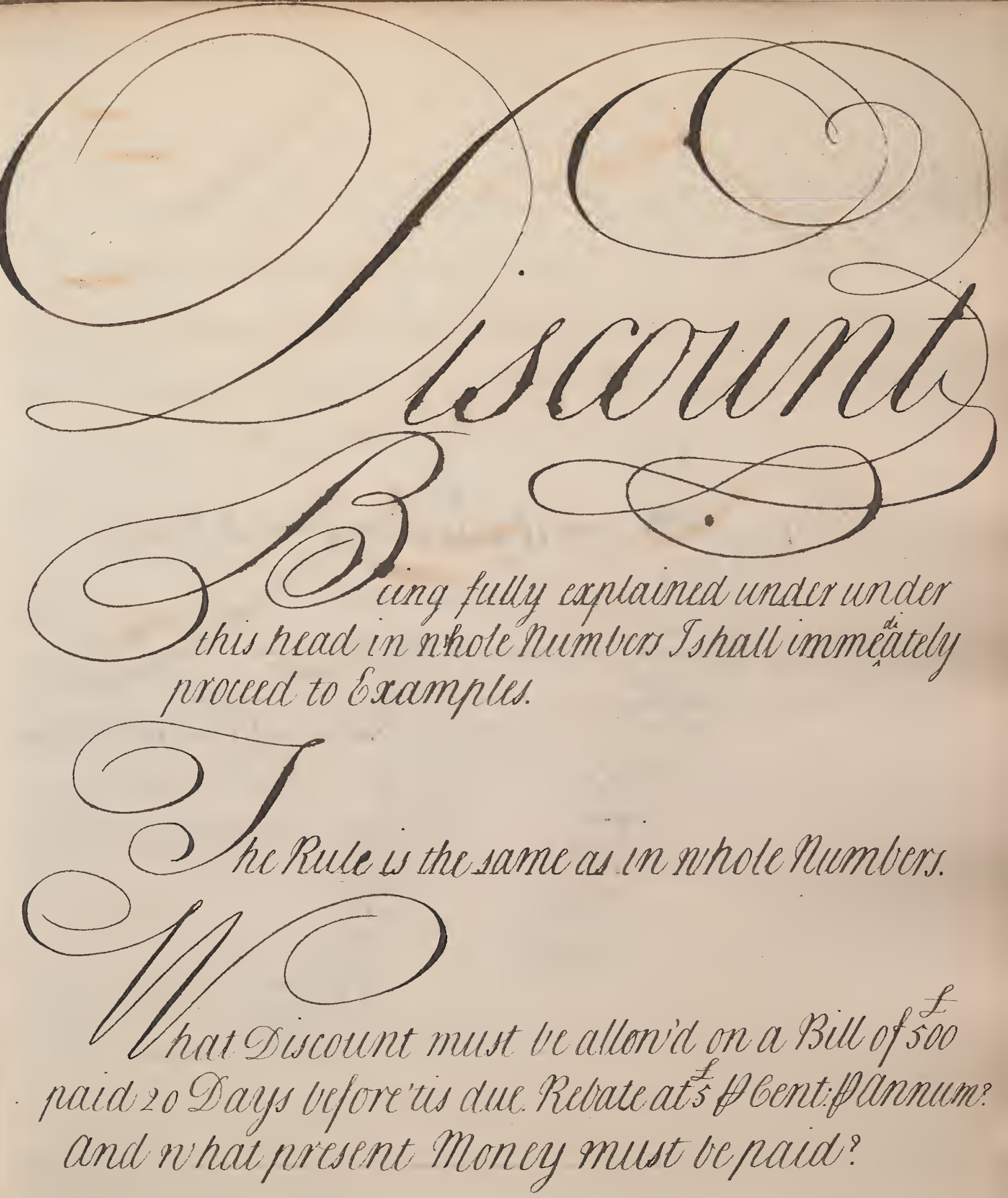




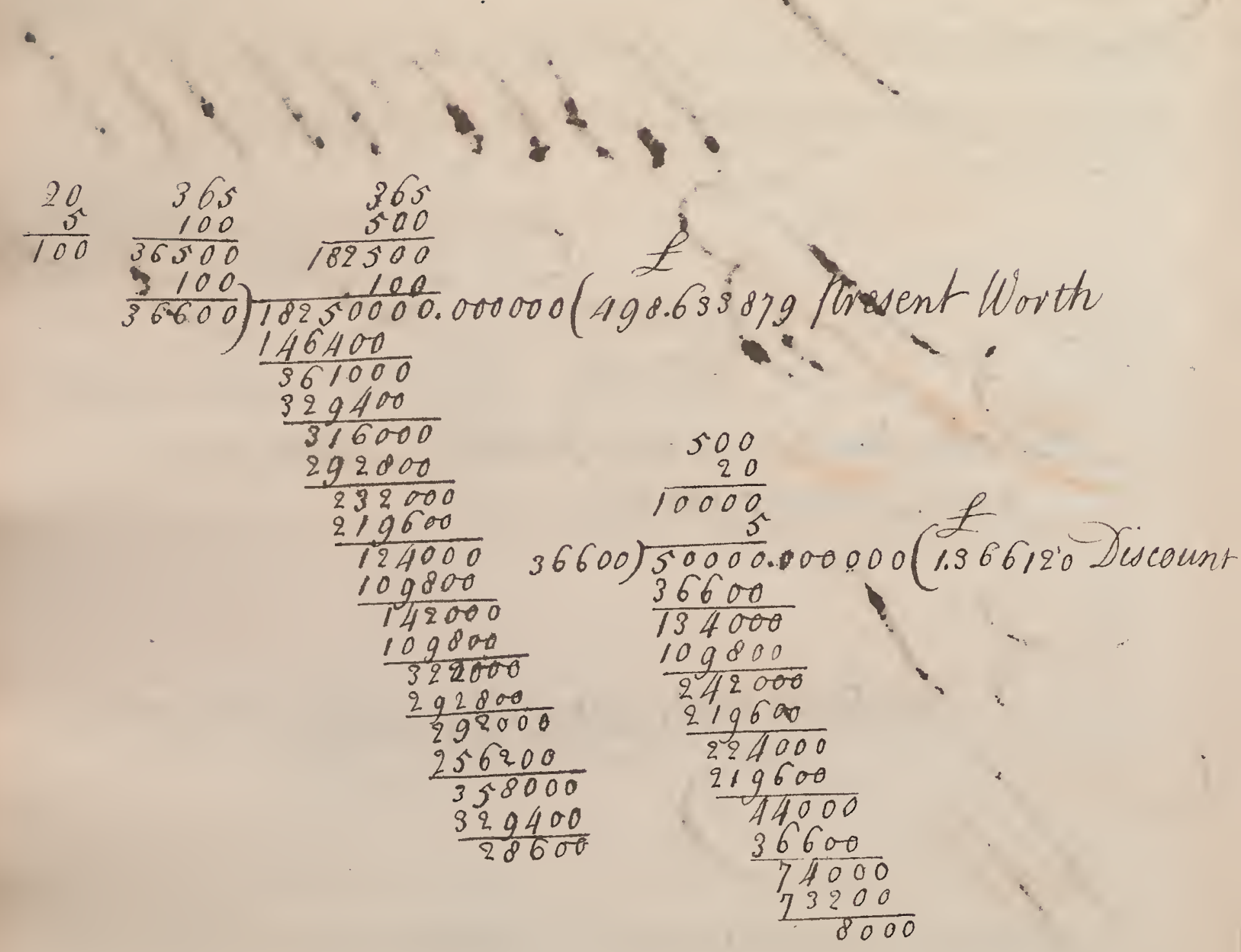




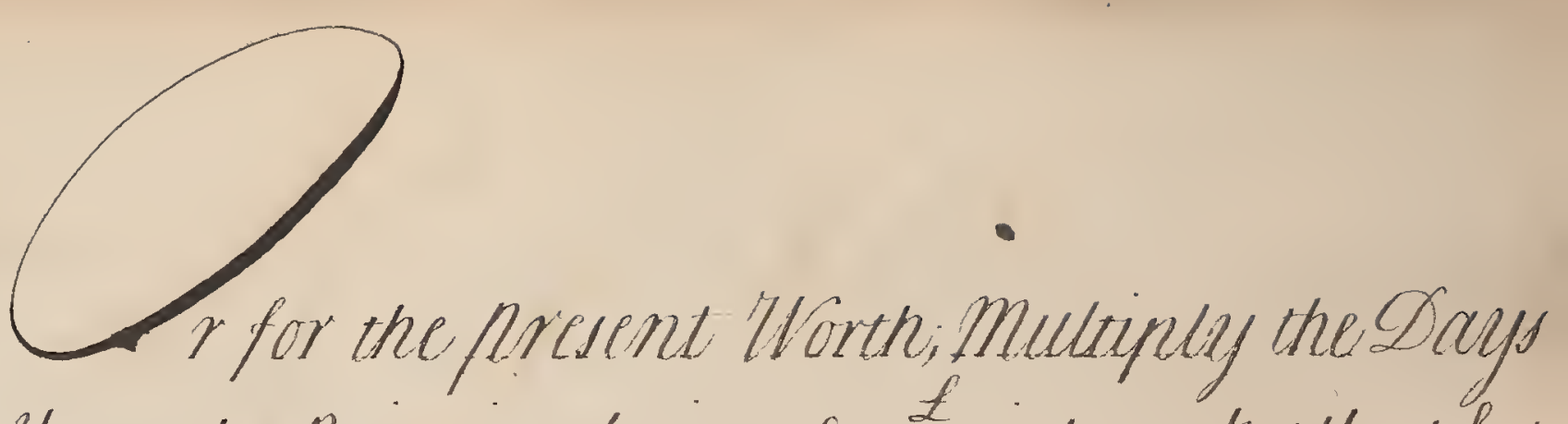
in a Year, the frincipal given, 8 10o into each other for a Dividend; s add the froduct of 365 ff 100 to that of the Dars multinlyed into the Rate given for a Divisor, \& the Lustiont resulting is the answer.
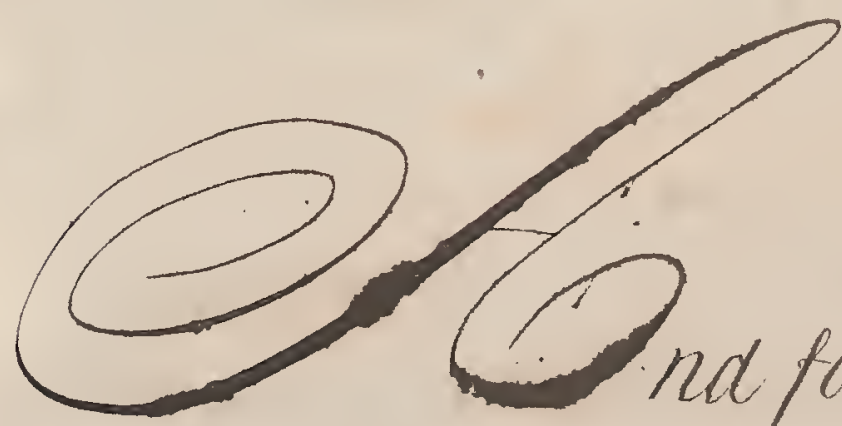
the Rate, Principal\& Days given together for aDivi. dend; tproceeding as above for a Divisor the 2uo= teent nill be the answer. 


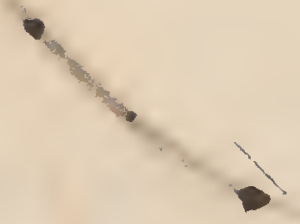

$+$

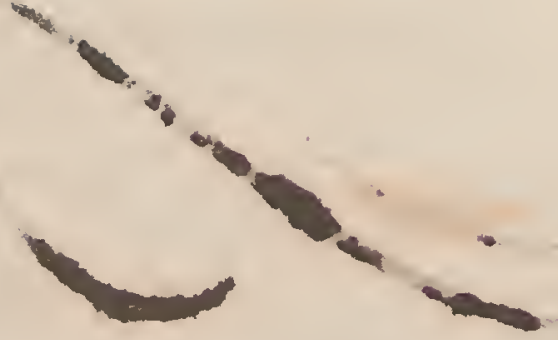



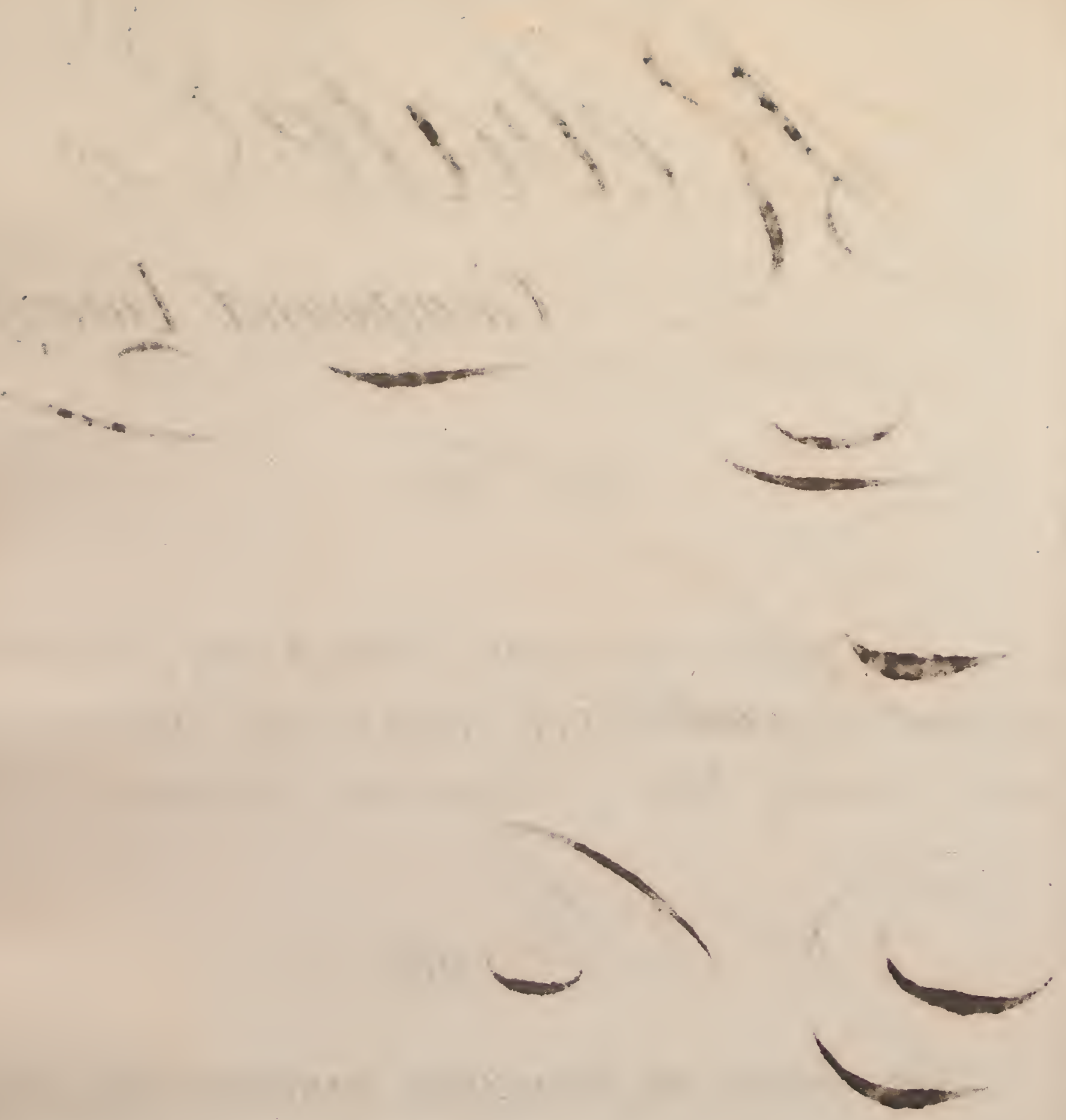


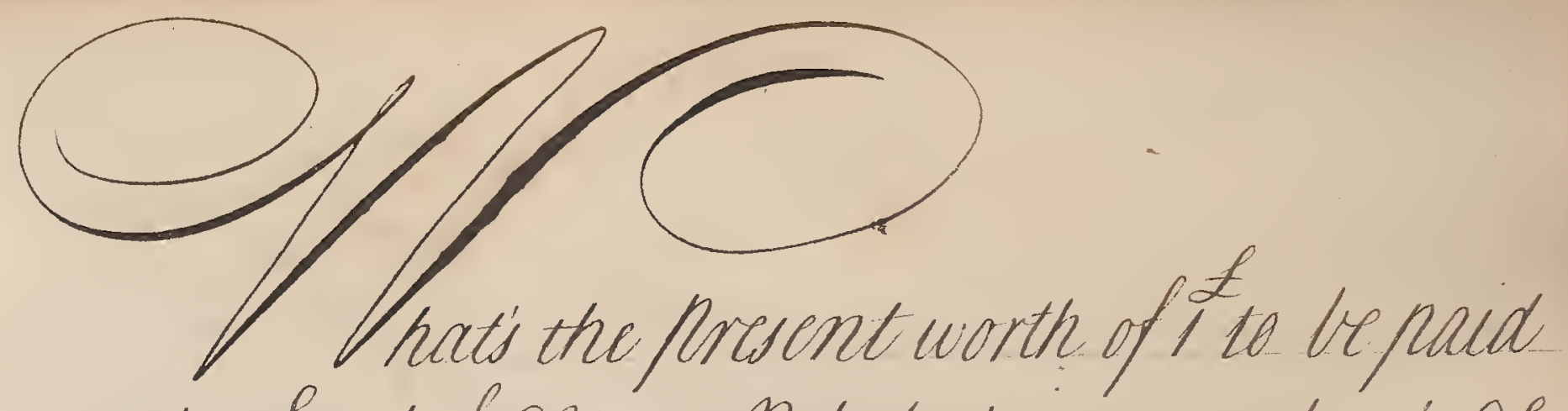
at the Gnd of byears Rebate leing made at 6\& pbont: H annum fompound Interest.

1.06) $1.000000000(1.06) .943396200(1.06) .80899964000) 1.06) .8396192000$

$$
\begin{aligned}
& \frac{954}{460} \\
& \frac{424}{360} \\
& \frac{310}{420} \\
& \frac{310}{1020} \\
& \frac{954}{660} \\
& \frac{636}{240} \\
& \frac{212}{20}
\end{aligned}
$$
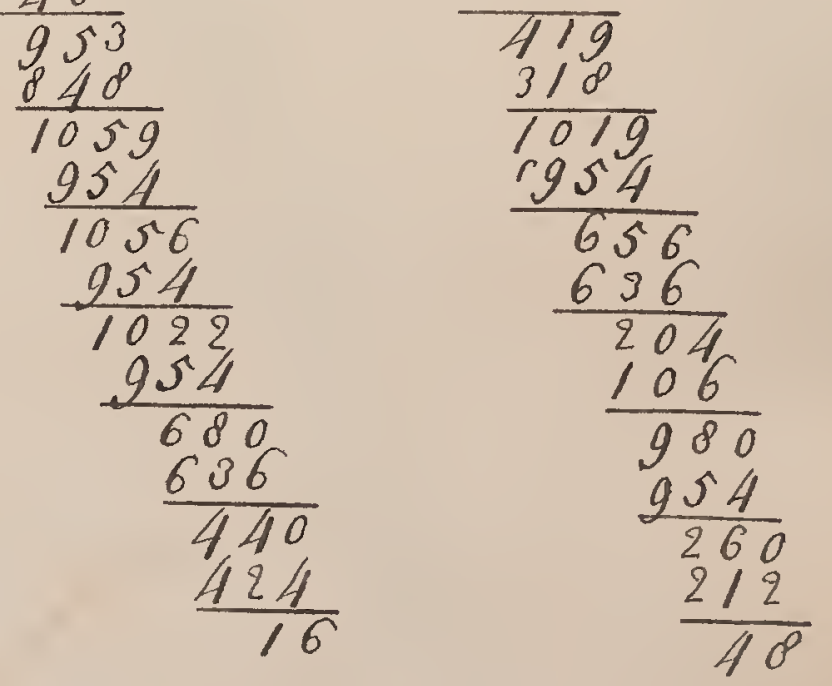

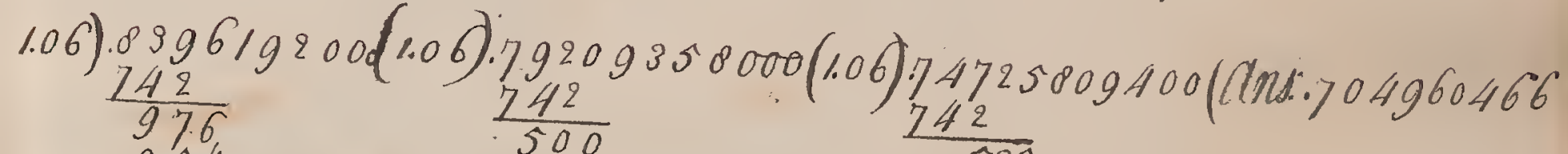

$$
\begin{aligned}
& \frac{242}{976} \\
& 254 \\
& \frac{212}{99^{2}} \\
& \frac{204}{380} \\
& \frac{310}{620} \\
& \frac{530}{900} \\
& \frac{048}{52} \\
& \frac{242}{500} \\
& \frac{424}{769} \\
& \frac{742}{273} \\
& \frac{2 / 2}{615} \\
& 500 \\
& 858 \\
& \frac{848}{1000} \\
& \frac{954}{460} \\
& \frac{424}{36}
\end{aligned}
$$

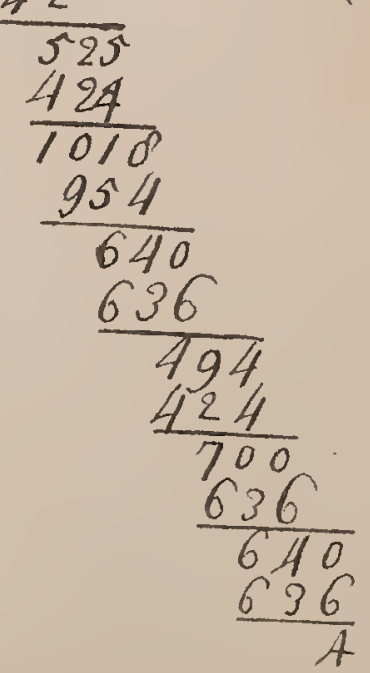




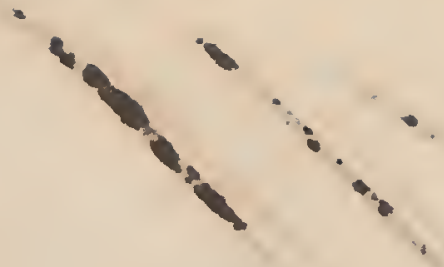




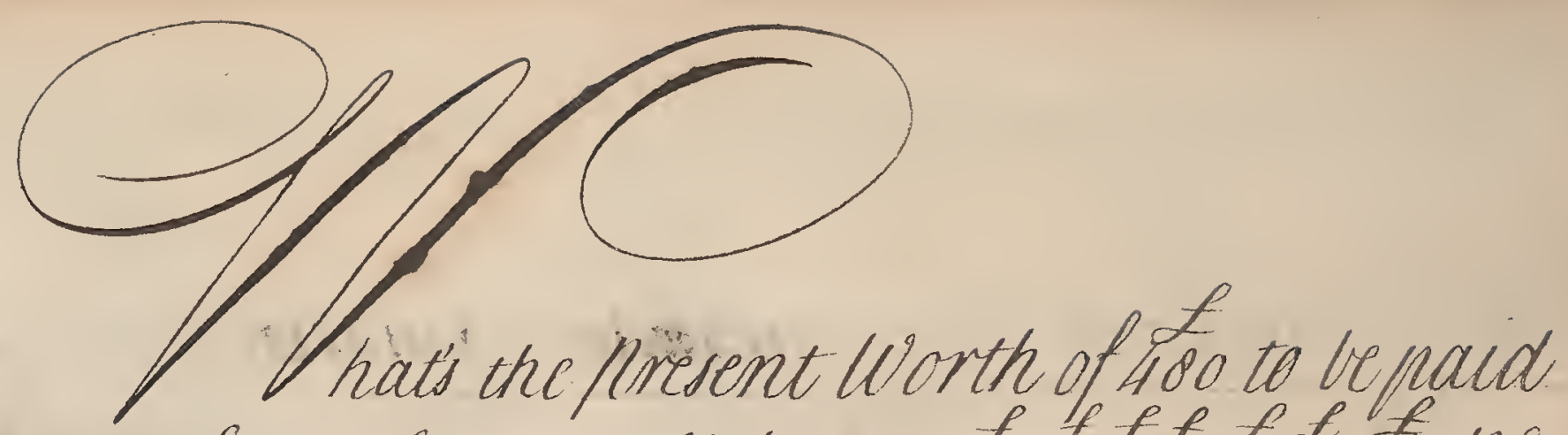

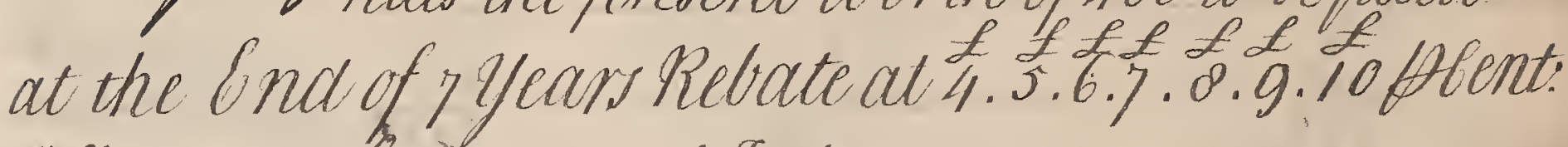
pernnum fompound Intercst?

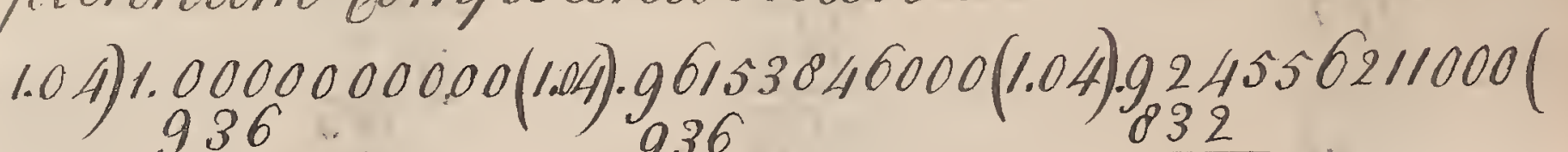

$$
\begin{aligned}
& \frac{936}{640} \\
& \frac{624}{160} \\
& \frac{104}{560} \\
& \frac{520}{400} \\
& \frac{312}{880} \\
& \frac{832}{480} \\
& \frac{416}{640} \\
& \frac{624}{16}
\end{aligned}
$$

1.04).

$$
\begin{aligned}
& \begin{array}{l}
\frac{936}{255} \\
\frac{200^{\circ}}{473} \\
\frac{416}{518} \\
\frac{520}{584} \\
\frac{320}{646} \\
\frac{624}{220} \\
\frac{208}{120} \\
\frac{104}{160}
\end{array} \\
& \begin{array}{l}
888996356701854004189 \\
\frac{032}{569} \\
\frac{320}{499} \\
\frac{416}{836} \\
\frac{832}{4335} \\
\frac{416}{196} \\
\frac{104}{923} \\
\frac{982}{050}
\end{array}
\end{aligned}
$$$$
\frac{104}{56}
$$$$
\begin{aligned}
& \frac{032}{925} \\
& \frac{932}{935} \\
& \frac{032}{1036} \\
& \frac{936}{1002} \\
& \frac{936}{661} \\
& \frac{624}{371} \\
& \frac{372}{190} \\
& \frac{520}{700} \\
& \frac{624}{760} \\
& \frac{728}{32}
\end{aligned}
$$ 


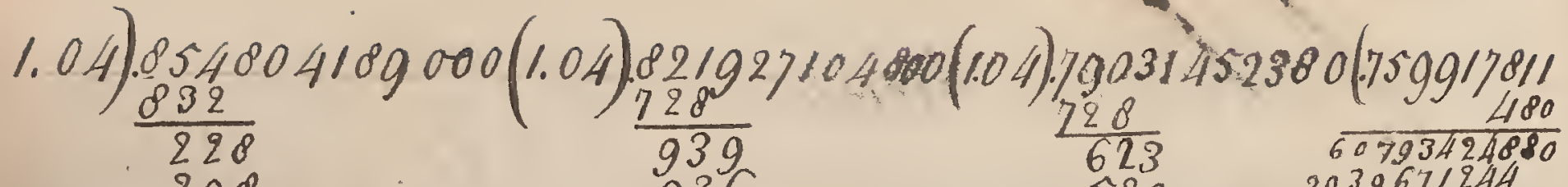

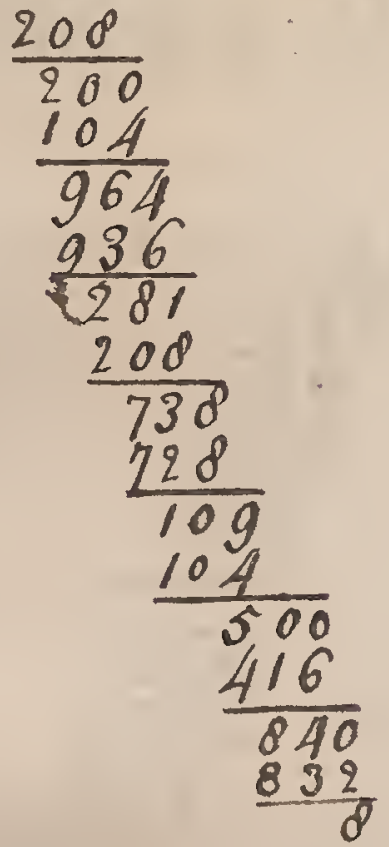

236

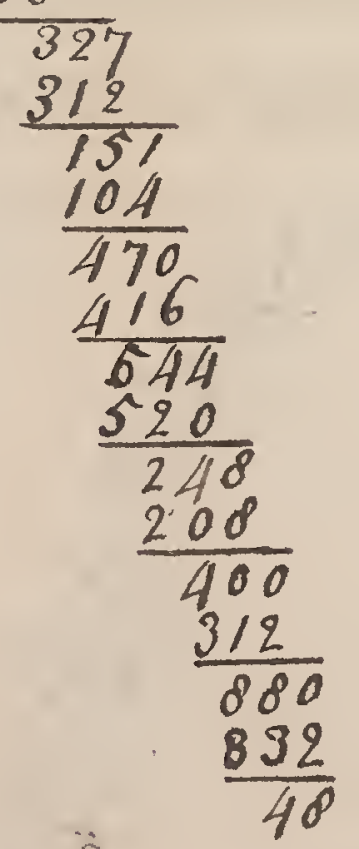

$\frac{520}{1034} \quad 2 \frac{3039671244}{364.960549280}$ 936 954 236 185

104

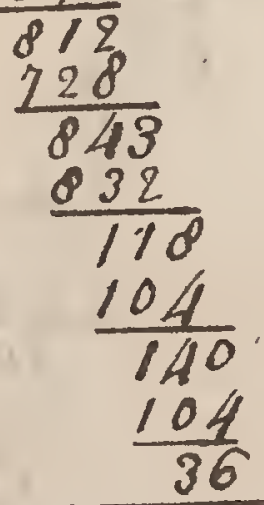

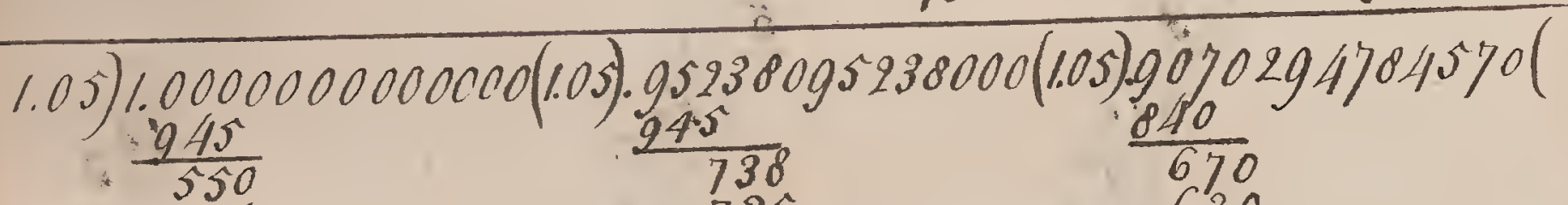

$\frac{525}{250}$

$\frac{210}{400}$

$\frac{315}{850}$

840

1000

945

550

$\frac{525}{250}$

210

$\frac{210}{400}$

$\frac{3 / 5}{850}$

$\frac{840}{10}$
738

735

309

210

$\frac{210}{95}$

945

502

420

823

235

880

840

480

420

600

525

750

$\frac{235}{15}$
670

630

402

$\frac{315}{879}$

840

394

315

797

$\frac{235}{628}$

525

1034

945

895

840

5.5\%

$\frac{525}{320}$ 
$1.05) \cdot 8638375985300(1.05) \cdot 8227024747900(1.05) .7839526166460$

$$
\begin{aligned}
& \frac{840}{238} \\
& \frac{210}{283} \\
& \frac{210}{737} \\
& \frac{235}{259} \\
& \frac{218}{49.8} \\
& \frac{420}{795} \\
& \frac{235}{503} \\
& \frac{420}{830} \\
& \frac{235}{950} \\
& \frac{245}{5}
\end{aligned}
$$

235
87
8

877

370

$3 / 5$

$5 \hat{s}^{2}$

525

274

$\frac{210}{647}$

$\frac{630}{174}$

$\frac{105}{697}$

$\frac{630}{679}$

630

490

$\frac{420}{700}$

$\frac{630}{70}$
735

420

652

630

226

$\frac{210}{167}$

$\frac{105}{566}$

$\frac{525}{116}$

$\frac{315}{1014}$

945

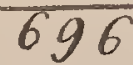

630 660 630 235

112

105

715

$\frac{630}{853}$

$\frac{840}{139}$

103

346

$\frac{315}{316}$

$3 / 5$

1000

$$
945
$$$$
56854506407400
$$$$
\text { 28842725320380 }
$$$$
f 341.127038445400
$$

$1.06) \frac{1000000000(9433962}{954}$ $\frac{954}{460}$ $\frac{424}{360}$

$3 / 8$

420

$\frac{318}{1020}$

$\frac{954}{660}$

$\frac{636}{240}$
$\frac{212}{28}$ 
$1.06) .943396200(106) .889996400(1.06) \cdot \frac{8396192000(79209358}{\frac{848}{953}}$
$\frac{8419}{976}$ 953

$$
\begin{aligned}
& \frac{848}{1059} \\
& \frac{954}{1056} \\
& \frac{954}{1022} \\
& \frac{954}{680} \\
& \frac{696}{440} \\
& \frac{424}{16}
\end{aligned}
$$

$\frac{318}{1019}$

954

656

$\frac{636}{204}$

106

980

$\frac{954}{260}$

$\frac{212}{48}$
976

954

221

$2 / 2$

$99^{2}$

954

$30^{\circ 0}$

$3 / 8$

620

$\frac{530}{900}$

$\frac{848}{52}$

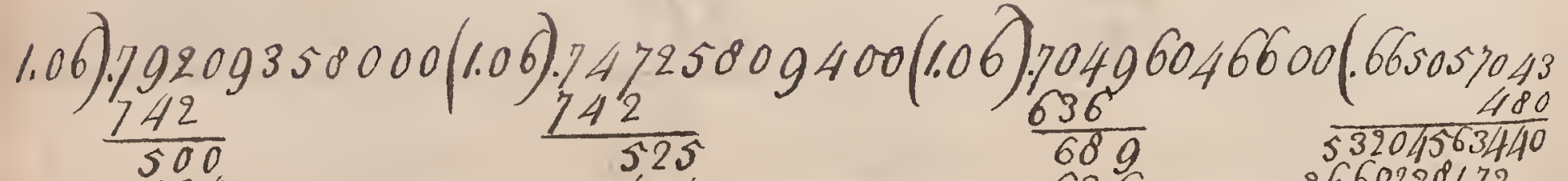

424

769

742

273

$\frac{212}{615}$

530

858

848

1000

$\frac{954}{460}$

$\frac{424}{36}$ $\frac{424 .}{1018}$

954

640

636
636

536 :

$\frac{530}{604}$

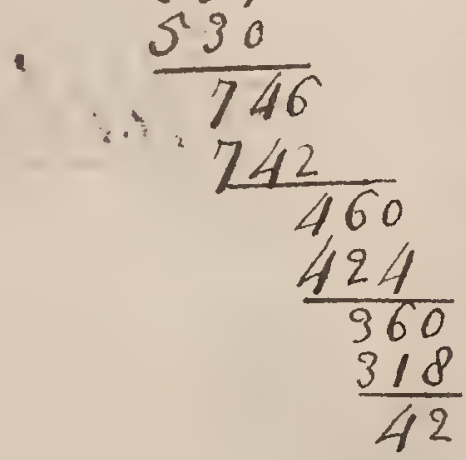

742 $f^{2660228172} 3 \frac{19.227380640}{3}$
700

636

640

$\frac{636}{7}$

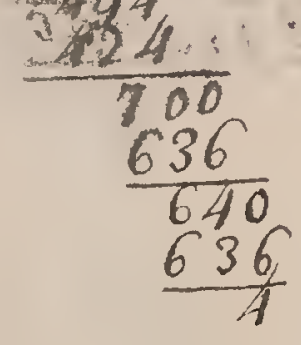




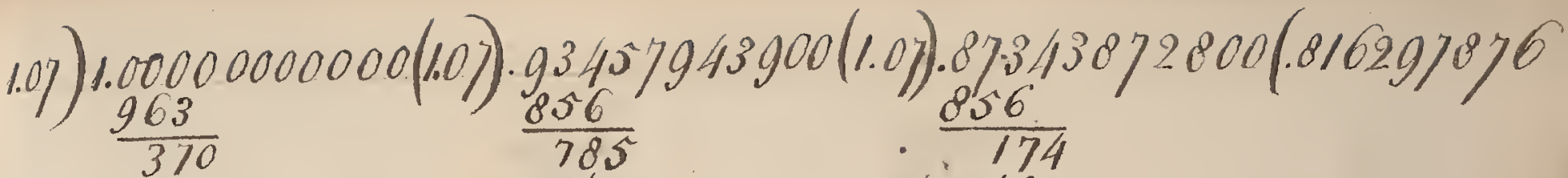
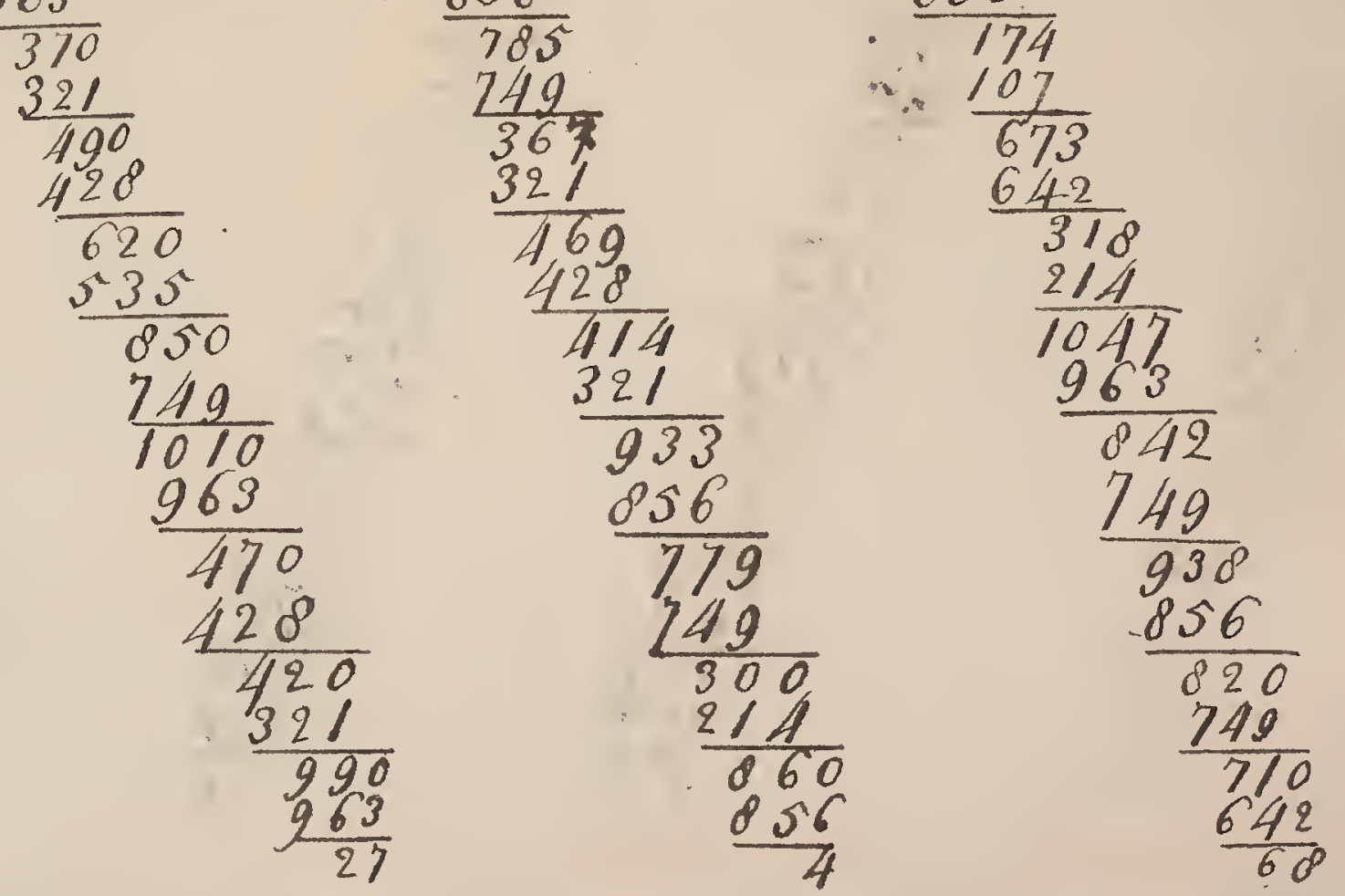

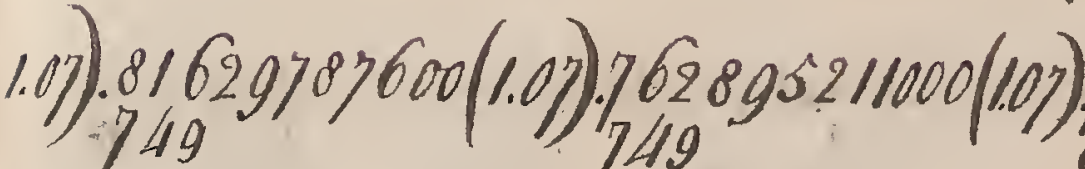
$\frac{149}{672}$ $\frac{642}{309}$ $\frac{749}{150}$

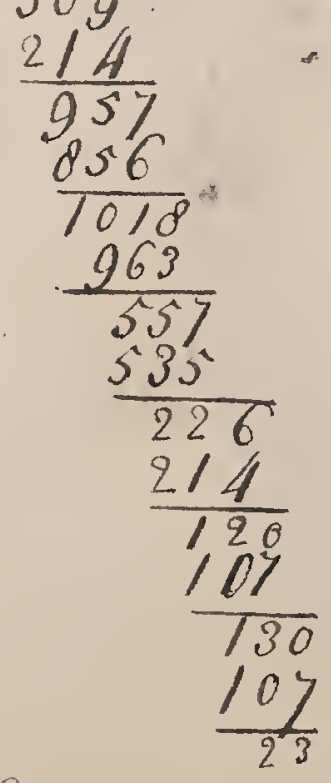

.622749441

49819979280

$7 / 29861905$

642

709

$\frac{10 \%}{319}$

$\frac{214}{1055}$

963

$\begin{array}{r}922 \\ 836 \\ \hline 661\end{array}$

642

191

$\frac{107}{840}$

$\frac{249}{910}$

856

540

$\frac{535}{5}$
642

$670^{\circ}$

642

366

321

447

428

237

214

230

$2 / 4$

245

214

$3 / 0$

214

960

056

1040

963
. 6003422289 642

24

294

214

802

249

532

$\frac{428}{1042}$

263

792

749

438

$\frac{428}{\log }$

$\frac{10 y}{2}$ 


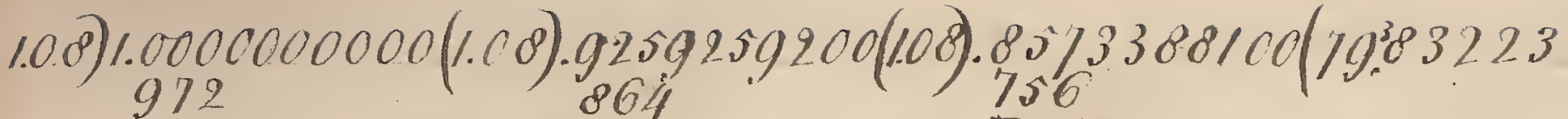
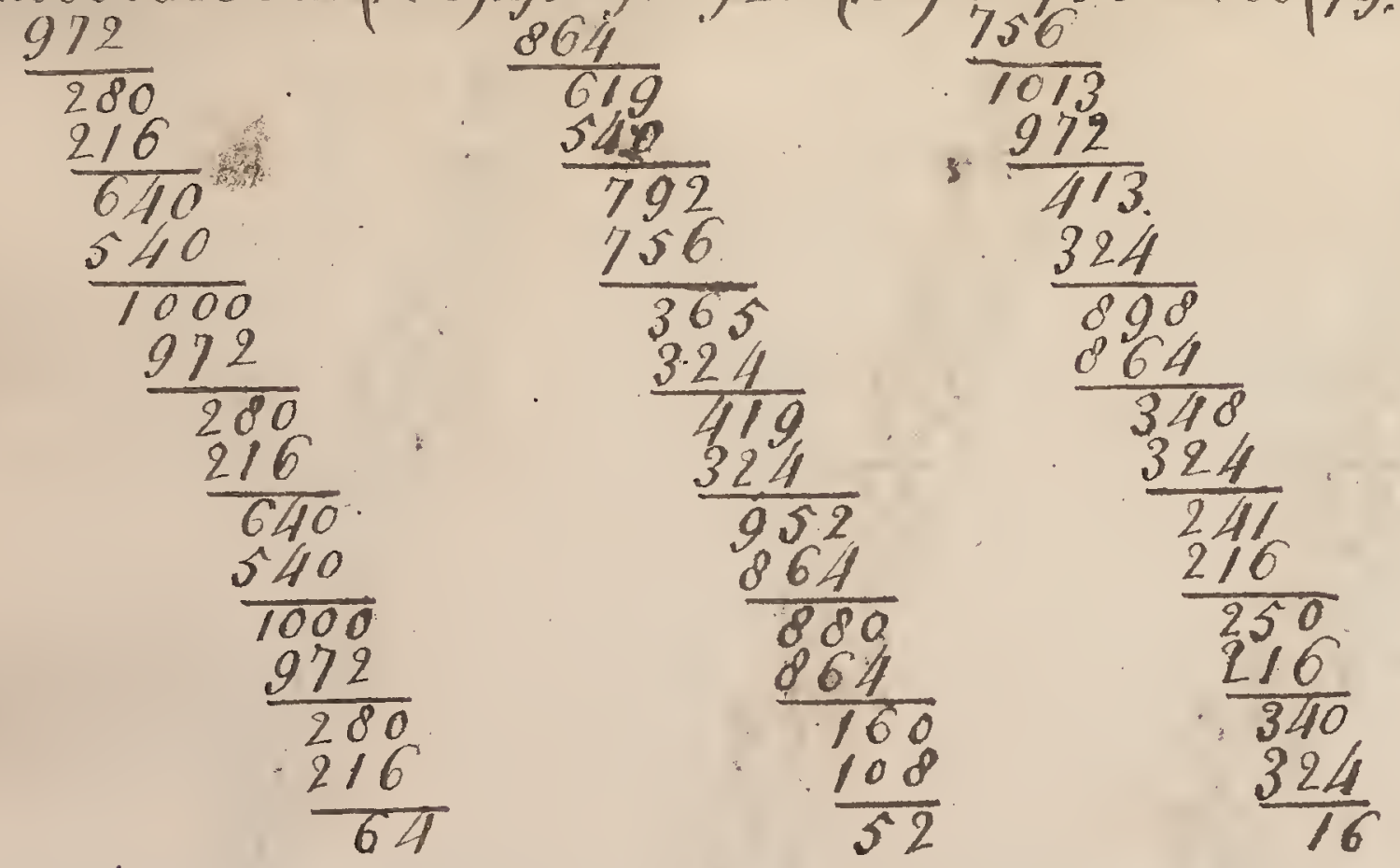

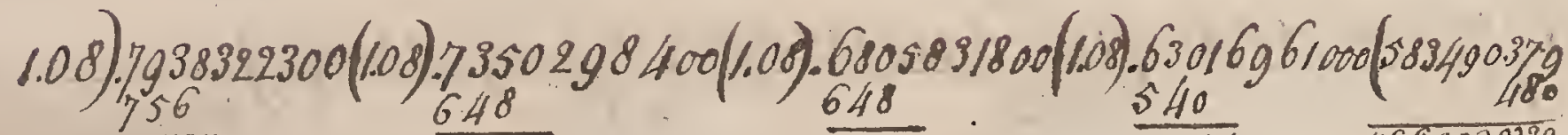

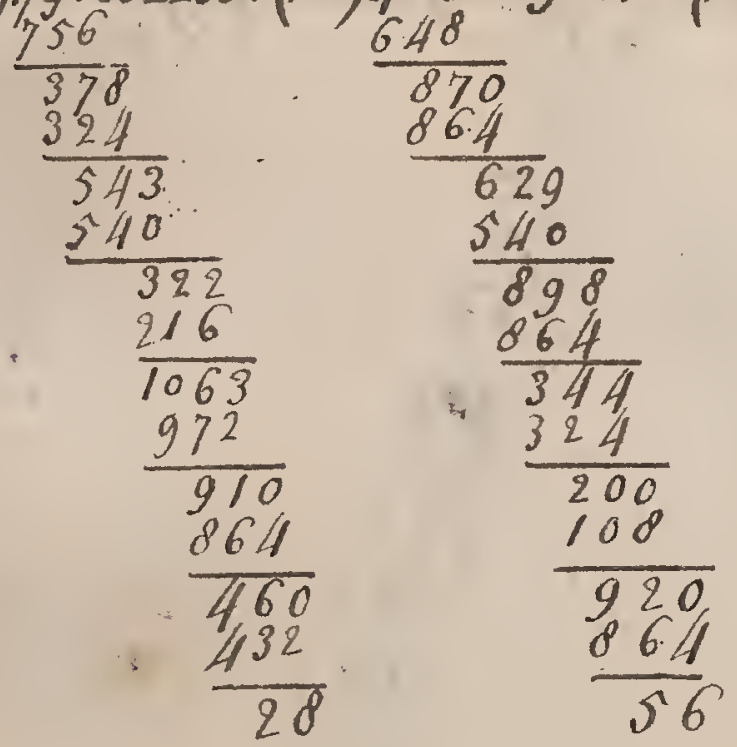

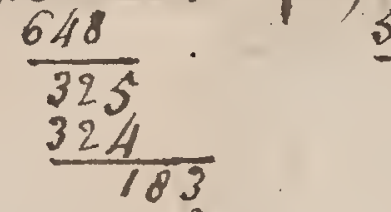

108

751

648

1038

972

660

648

120

108
$9 0 1 \quad \longdiv { 4 6 6 9 9 2 3 0 3 2 0 }$ 8642333961516

$976 \neq 20004531920$

972

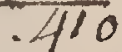

324

860

$\frac{756}{1040}$

$\frac{972}{68}$ 
$\because \cdots \quad \mathcal{L}$

The Principat 1.

OH

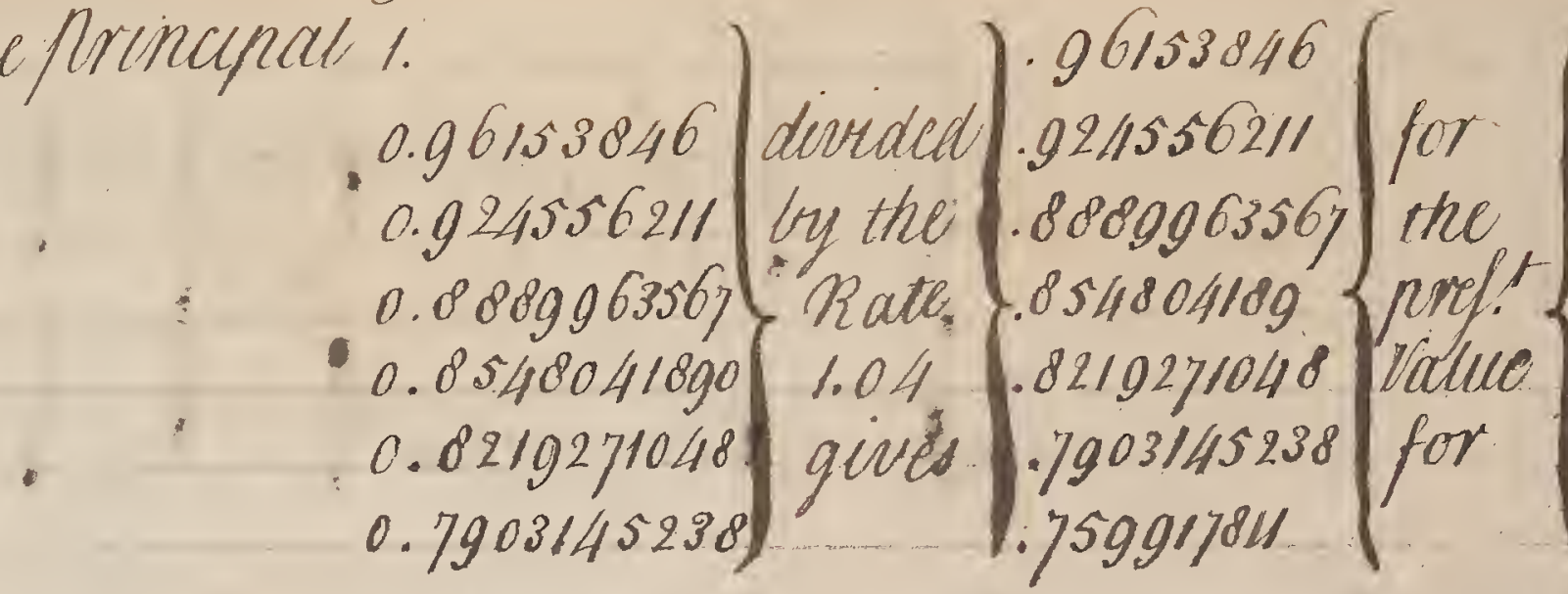

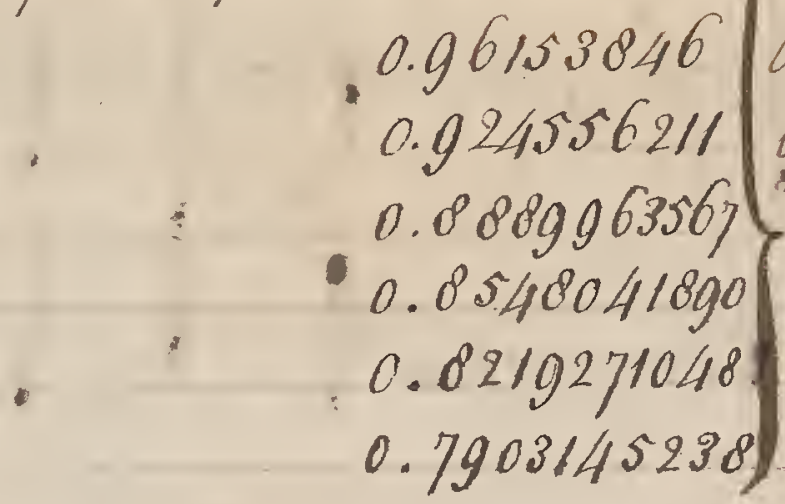

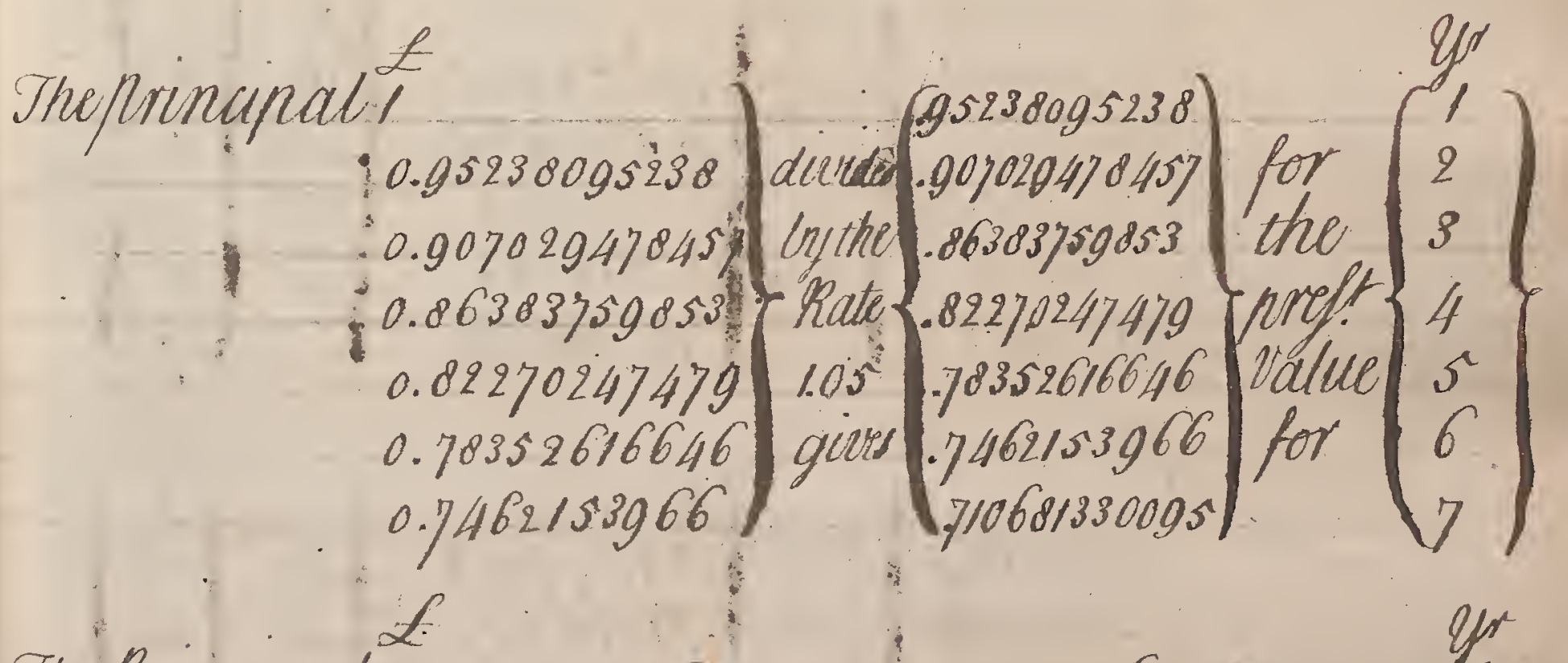

The Principal .

0.9433962

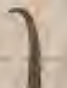

0.0899964 ty the

.9433962

0.0396192 rate $0.79209358 \mid 1.06$ 0.747250094 gives $0.704960466)$ $\left.\begin{array}{l}1.06 \\ \text { gives }\left(\begin{array}{l}.797250094 \\ 704960466 \\ 6.65057043\end{array}\right.\end{array}\right\}$ for 3 4 5 71 


\section{The Arinipal'}

0.934579439

\section{The Principals}

\section{The priminats}

\begin{tabular}{l|l}
0.92592592 & dinided \\
0.05733881 & .05733801 \\
0.753 the & 79383223
\end{tabular} 0.79383223

\subsection{68058318 $0.63016 \mathrm{~g} 61$

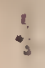

0.91743119 divided 0.84169999 In the 0.77218347 . Trate 70.84252 0.7004252 0.64993137 0.59626731 lic $6499313 \%$

(-91743 119 .84169999 .77218341 The prinipalt

0.909090909 distade
0.82644620
0.7513148 (b)

(909090909 .82641628 .9513148 0.7513148 0.6830134 0.62092129 0.56447388

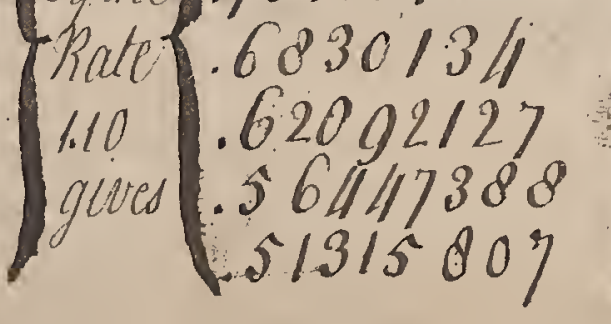

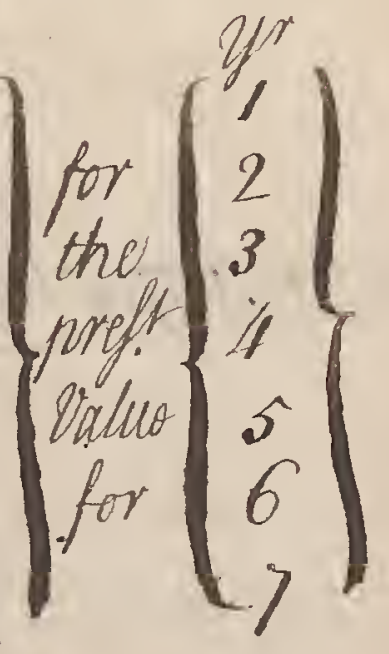


Table st the Amt of if for anyn: of $y$ ps $u$ inder 33 at the hates of $5 e \cdot 6$ beent thannum fompound Interest. Table 20 The amount of 12 Annuity for any n. of y. under 33 at the prates of swe Abont. Hnnum fompound Interest.

\begin{tabular}{|c|c|c|c|c|}
\hline \multicolumn{2}{|c|}{ Rates } & Years & $s$ & 6 \\
\hline $\begin{array}{l}1.05000 \\
1.10250 \\
1.15962 \\
1.21550 \\
1.27628 \\
1.34009 \\
1.40710 \\
1.47745\end{array}$ & $\begin{array}{l}1.06000 \\
1.12360 \\
1.19101 \\
1.26249 \\
1.33822 \\
1.41852 \\
1.50363 \\
1.59384\end{array}$ & $\begin{array}{l}1 \\
2 \\
3 \\
4 \\
5 \\
6 \\
7 \\
8\end{array}$ & $\begin{array}{l}1.00000 \\
2.05000 \\
3.15250 \\
4.31012 \\
5.52563 \\
6.8019 \% \\
0.14200 \\
9.54910\end{array}$ & $\begin{array}{l}1.00000 \\
2.06000 \\
3.18360 \\
4.37461 \\
5.63709 \\
6.97532 \\
8.39383 \\
9.89746\end{array}$ \\
\hline $\begin{array}{l}1.55132 \\
1.62809 \\
1.71034 \\
1.79585 \\
1.80560 \\
1.97993 \\
2.07992 \\
2.18289\end{array}$ & $\begin{array}{l}1.68948 \\
1.79084 \\
1.89829 \\
2.01219 \\
2.13292 \\
2.26090 \\
2.39655 \\
2.54035\end{array}$ & $\begin{array}{l}9 \\
10 \\
11 \\
12 \\
13 \\
14 \\
15 \\
16\end{array}$ & $\begin{array}{l}11.02656 \\
12.57789 \\
14.20678 \\
15.91712 \\
17.71298 \\
19.59863 \\
21.57856 \\
23.65949\end{array}$ & $\begin{array}{l}11.49131 \\
13.18079 \\
14.97164 \\
16.86994 \\
18.88219 \\
21.01506 \\
23.27597 \\
25.67252\end{array}$ \\
\hline $\begin{array}{l}2.29201 \\
2.40662 \\
2.52695 \\
2.65329 \\
2.78596 \\
2.92526 \\
3.07 .152 \\
3.22510\end{array}$ & $\begin{array}{l}2.69277 \\
2.85434 \\
3.02559 \\
3.20713 \\
3.39956 \\
3.60353 \\
3.81975 \\
1.04893\end{array}$ & $\begin{array}{l}17 \\
10 \\
19 \\
20 \\
21 \\
22 \\
23 \\
24\end{array}$ & $\begin{array}{l}25.04036 \\
28.13238 \\
30.53200 \\
33.06595 \\
35.71925 \\
38.50521 \\
41.43047 \\
44.50199\end{array}$ & $\begin{array}{l}28.21288 \\
30.90565 \\
33.75999 \\
36.79599 \\
39.99272 \\
43.39929 \\
16.99582 \\
50.89057\end{array}$ \\
\hline $\begin{array}{l}3.38635 \\
3.55567 \\
3.73345 \\
3.92013 \\
4.11613 \\
4.32194 \\
4.53804 \\
4.76494\end{array}$ & $\begin{array}{l}4.29187 \\
4.54938 \\
4.82934 \\
5.11168 \\
5.41838 \\
5.74349 \\
6.08810 \\
6.45338\end{array}$ & $\begin{array}{l}25 \\
26 \\
27 \\
28 \\
29 \\
30 \\
31 \\
32\end{array}$ & $\begin{array}{l}47.72709 \\
51.11345 \\
54.66912 \\
58.40258 \\
62.32971 \\
66.43884 \\
70.76099 \\
55.29883\end{array}$ & $\begin{array}{l}54.86451 \\
59.15638 \\
63.70576 \\
68.52811 \\
73.63979 \\
79.05818 \\
84.80167 \\
90.08979\end{array}$ \\
\hline
\end{tabular}




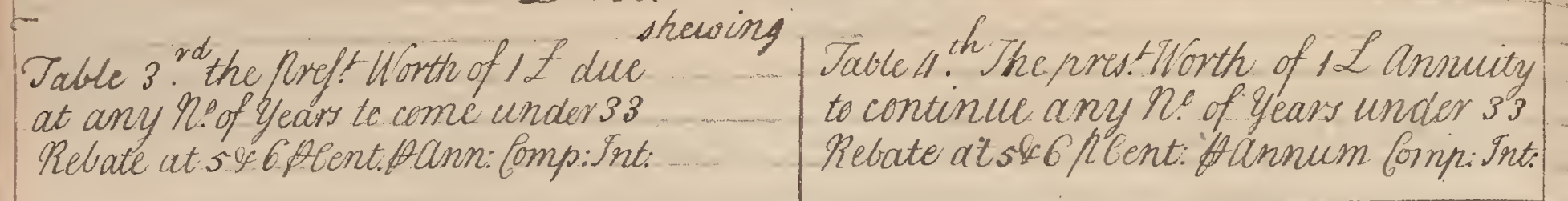

\begin{tabular}{|c|c|c|c|c|}
\hline \multicolumn{2}{|c|}{ Rates } & \multirow{2}{*}{ Years } & \multicolumn{2}{|c|}{ Rates } \\
\hline & $b$ & & 3 & $b$ \\
\hline $\begin{array}{l}.952381 \\
.907030 \\
.863838 \\
.822702 \\
.783526 \\
.416215 \\
.710682 \\
.676039\end{array}$ & $\begin{array}{l}.943396 \\
.889996 \\
.839619 \\
.792093 \\
.747580 \\
.704960 \\
.665057 \\
.627412\end{array}$ & $\begin{array}{l}1 \\
2 \\
3 \\
4 \\
5 \\
6 \\
7 \\
8\end{array}$ & $\begin{array}{l}0.95238 \\
1.85941 \\
2.72324 \\
3.54590 \\
4.32947 \\
3.07569 \\
5.78639 \\
6.46321\end{array}$ & $\begin{array}{l}0.94339 \\
1.83339 \\
2.03309 \\
3.46510 \\
4.21236 \\
4.91932 \\
5.18238 \\
6.20979\end{array}$ \\
\hline $\begin{array}{l}.64609 \\
.613913 \\
.504679 \\
50637 \\
230321 \\
505068 \\
401017 \\
.458111\end{array}$ & $\begin{array}{l}.591898 \\
.558394 \\
.526787 \\
.496969 \\
.468039 \\
.442301 \\
.417265 \\
.393647\end{array}$ & $\begin{array}{l}9 \\
10 \\
11 \\
12 \\
13 \\
14 \\
15 \\
16\end{array}$ & $\begin{array}{l}7.10782 \\
7.72173 \\
0.30641 \\
8.06325 \\
9.39357 \\
9.89864 \\
10.37965 \\
10.89777\end{array}$ & $\begin{array}{l}6.80169 \\
7.36008 \\
7.08687 \\
8.38384 \\
8.85268 \\
9.29498 \\
9.71225 \\
10.10589\end{array}$ \\
\hline $\begin{array}{l}.436296 \\
.415520 \\
.395934 \\
.376889 \\
358942 \\
.341849 \\
.325579 \\
.310067\end{array}$ & $\begin{array}{l}.371964 \\
.350343 \\
.330513 \\
.311804 \\
.294150 \\
.277505 \\
2261797 \\
.246978\end{array}$ & $\begin{array}{l}17 \\
18 \\
19 \\
20 \\
21 \\
22 \\
23 \\
24\end{array}$ & $\begin{array}{l}11.27406 \\
11.60958 \\
12.08332 \\
12.46291 \\
12.02115 \\
13.16300 \\
13.40857 \\
13.99864\end{array}$ & $\begin{array}{l}10.47726 \\
10.82760 \\
11.15811 \\
11.46992 \\
11.76400 \\
12.04158 \\
12.30338 \\
12.55035\end{array}$ \\
\hline $\begin{array}{l}.295302 \\
.281240 \\
.267848 \\
.25093 \\
.242946 \\
.231377 \\
.220389 \\
.209865\end{array}$ & $\begin{array}{l}.232998 \\
.219810 \\
.207368 \\
.195630 \\
.184456 \\
.174110 \\
.164254 \\
.154956\end{array}$ & $\begin{array}{l}25 \\
26 \\
27 \\
28 \\
29 \\
30 \\
31 \\
3.2\end{array}$ & $\begin{array}{l}14.09394 \\
14.37510 \\
14.64303 \\
14.09812 \\
15.14107 \\
15.37245 \\
15.59281 \\
15.00267\end{array}$ & $\begin{array}{l}12.78331 \\
13.00316 \\
13.21053 \\
13.40616 \\
13.09072 \\
13.76403 \\
13.92908 \\
14.08404\end{array}$ \\
\hline
\end{tabular}





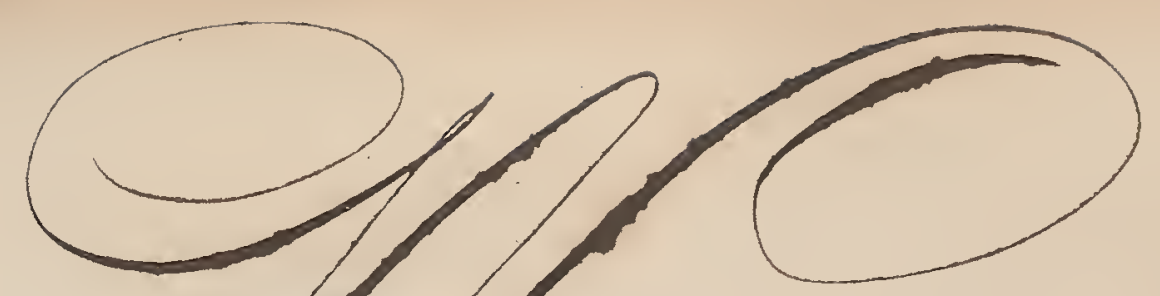

hat's the present Worth of a

of yof to continue slyears Rebate at sf flbent. plann.

$\mathcal{L}^{.95238093238} 70$
66.66666666660

63.49206349199

60.46863189710

57.58917323530

\& $\frac{54.84683165220}{303 \cdot 06336694319}$ Ansiler.

$f^{.82270247479} \frac{79832616646}{70}$ 



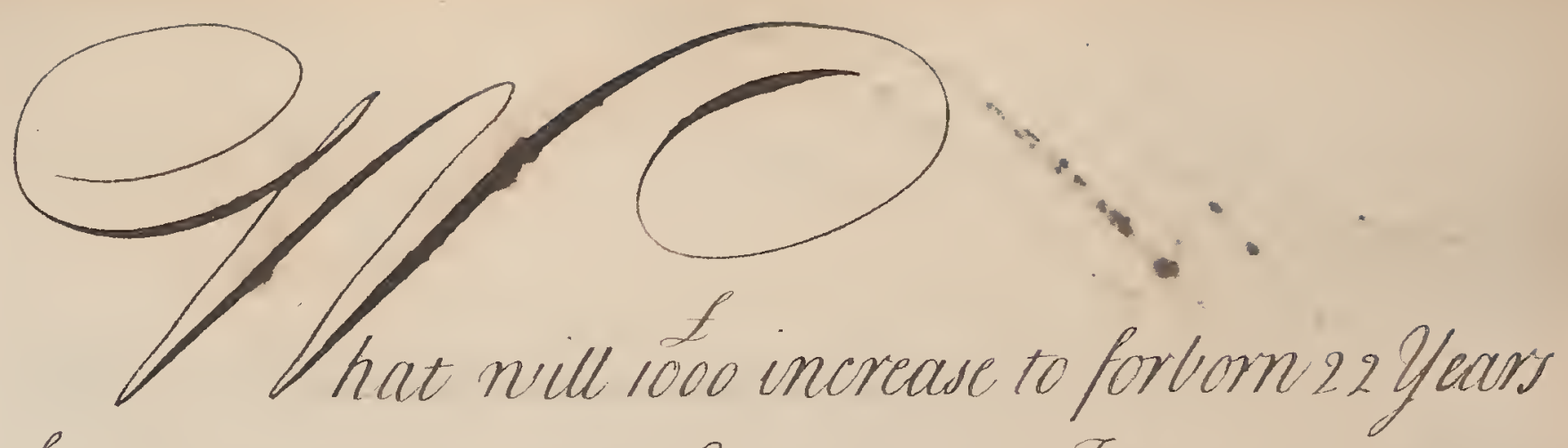

at sf bent: D A nnum bompound Interest?

1000

$\frac{1.05}{1050.00}$

$\frac{1.05}{5250}$

1050

1102.50

$\frac{1.05}{55 / 25}$

11025

1157.625

1.05

$5788 / 25$

1157625

1215.50625

$\frac{1.05}{60775312}$

12155062

$\overline{1216.28 / 512}$

$\frac{1.05}{638 / 40755}$

127628151

$\longdiv { 1 3 4 0 . 0 9 5 5 8 5 5 }$

$\frac{1.05}{6700477925} \cdot \frac{1.05}{9899657570}$ $\frac{1340095585}{1407.100364252078 .92808970}$ $147 \% .453382$ 1.05 7387276910 1477455382

$\longdiv { 1 5 5 1 . 3 2 8 1 5 1 1 0 }$

$\overline{756640755}$

1551328151

1628.09455855

$\frac{1.05}{8144472790}$

1628894558

1710.33928590

8551696425

1) 10339285

1795.85624925

1.05

8979287245

1795056249

1805.64906145

$\frac{1.05}{9428245305}$

1885649061

$\longdiv { 1 9 7 9 . 9 3 1 5 1 4 0 5 }$

1.05

$\frac{1.05}{7035501820}$

$\frac{1407100364}{1477.45538220} \frac{2078928089}{2182.87449345}$ 


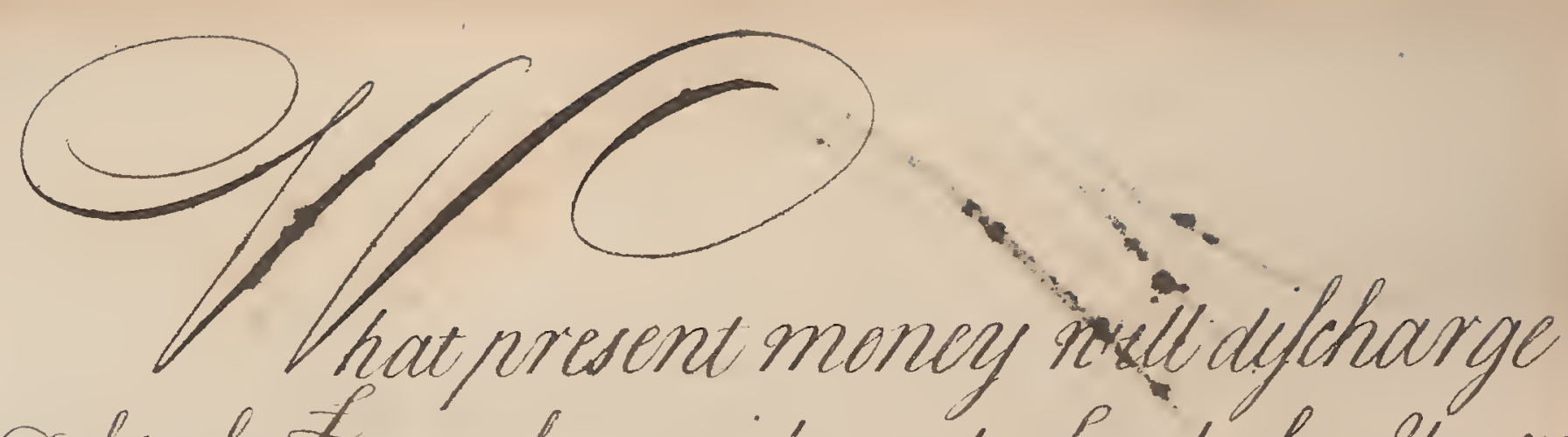

aDele of is oo to be paid at the bid of 10 Years, relate being made at sf flont.panniom for pound Interest?

.613913

$\frac{7500}{306956500}$

4293391

f 4604347500 answer. 


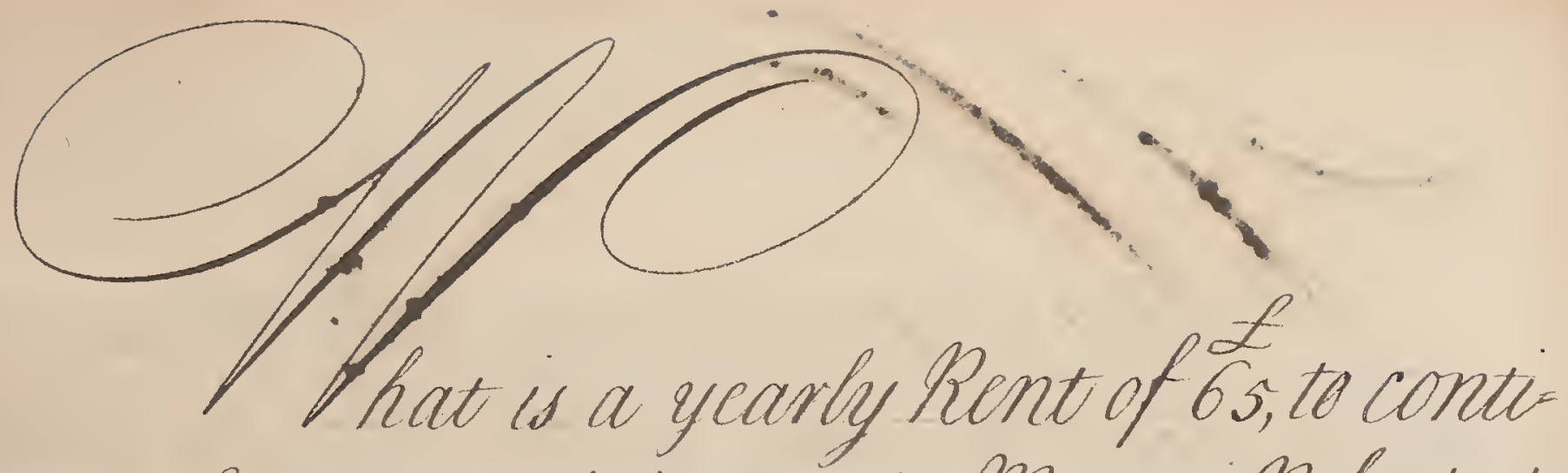
nue so Years, north in ready Money Reliato tor ing made at 6.fflent.ff Cinn:formpound Snterest?

$$
\begin{array}{r}
13.76483 \\
\frac{65}{6802415} \\
2 \frac{8258898}{894.71395} \text { unswer } \\
\hline
\end{array}
$$


11.16992

$\frac{500}{573496000}$

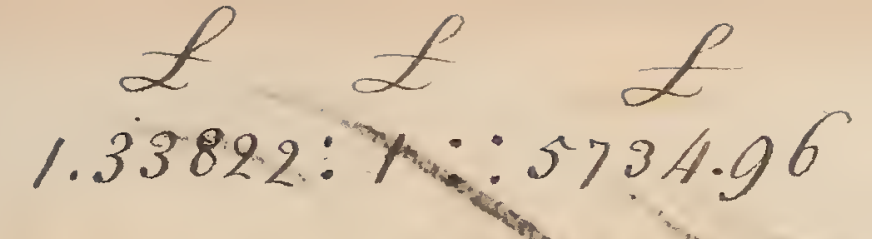

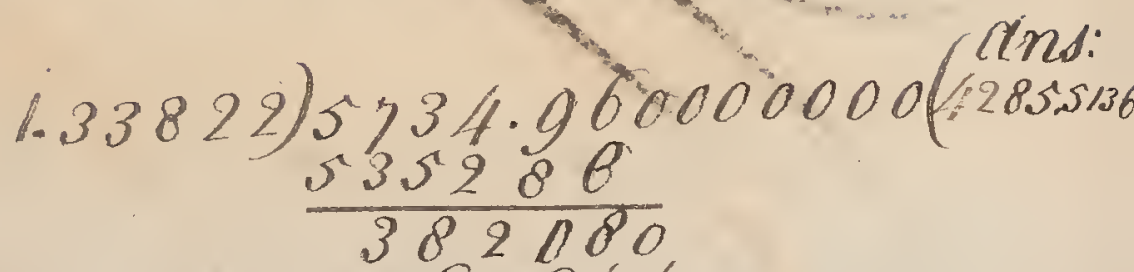

4.21236

$\frac{267644}{1114360}$

$\frac{1070576}{737840}$

$\frac{669110}{607300}$

$\frac{669110}{181900}$

$\frac{133822}{480780}$

$\frac{380466}{923140}$

$\frac{868932}{44208}$ 


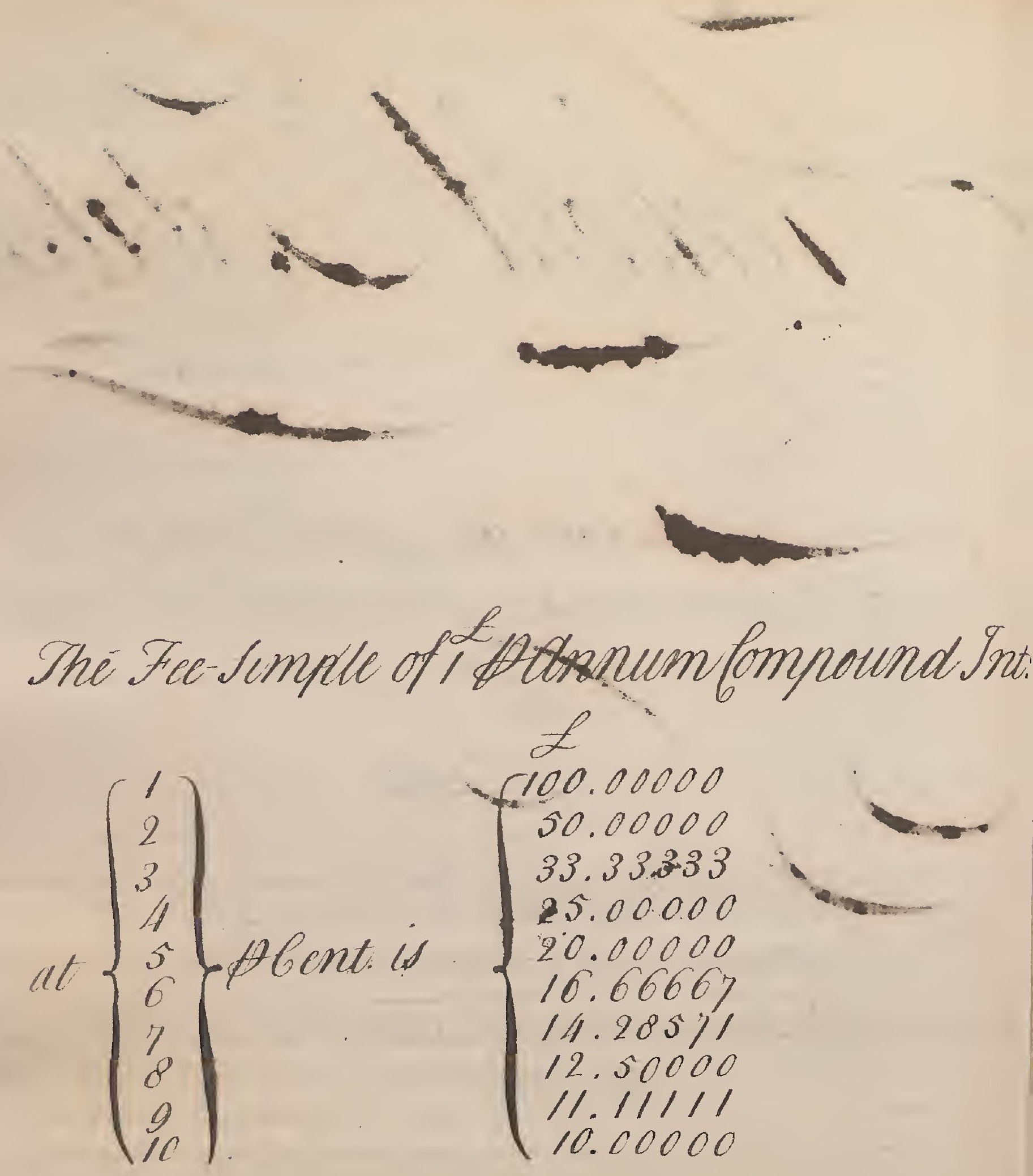




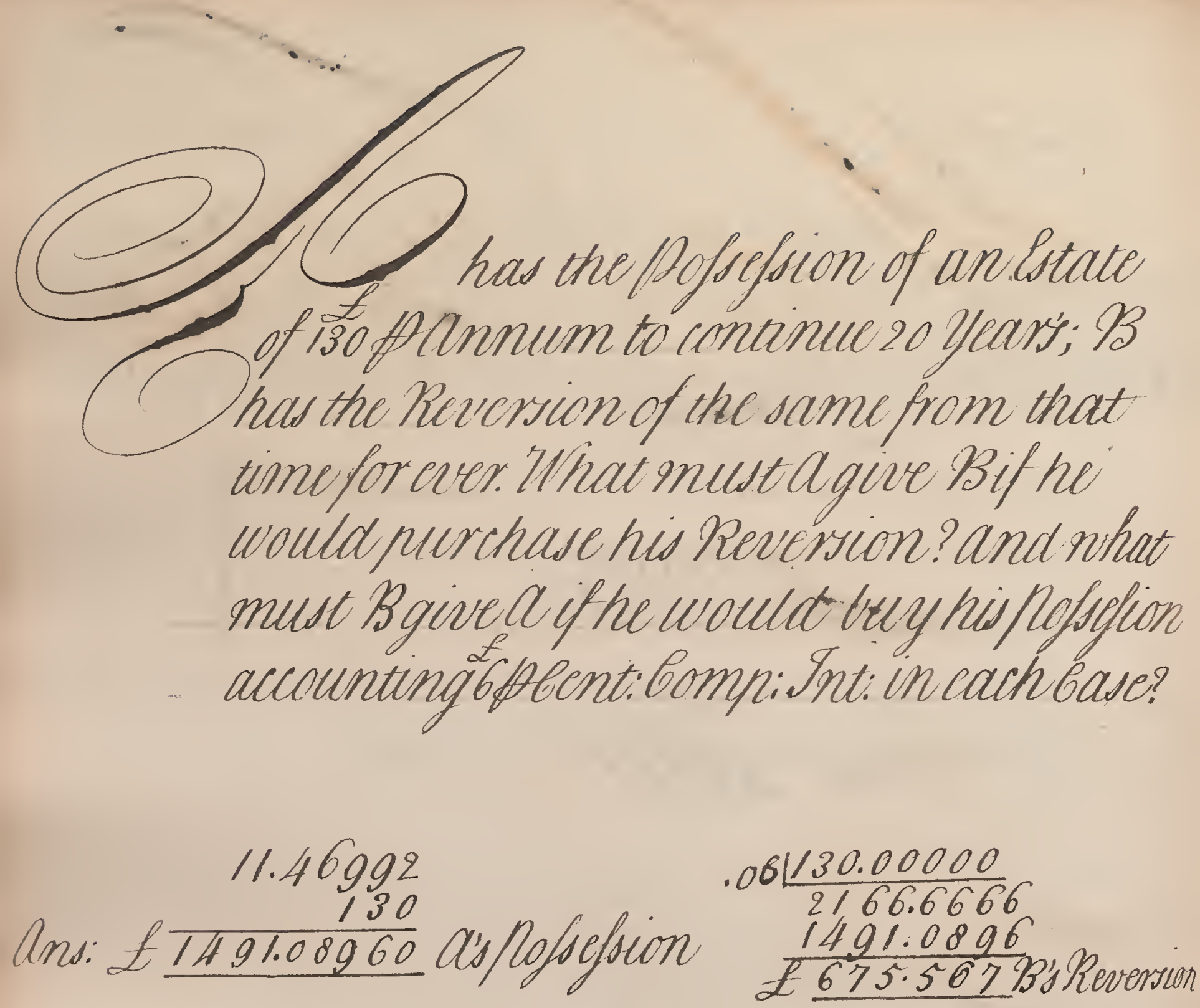




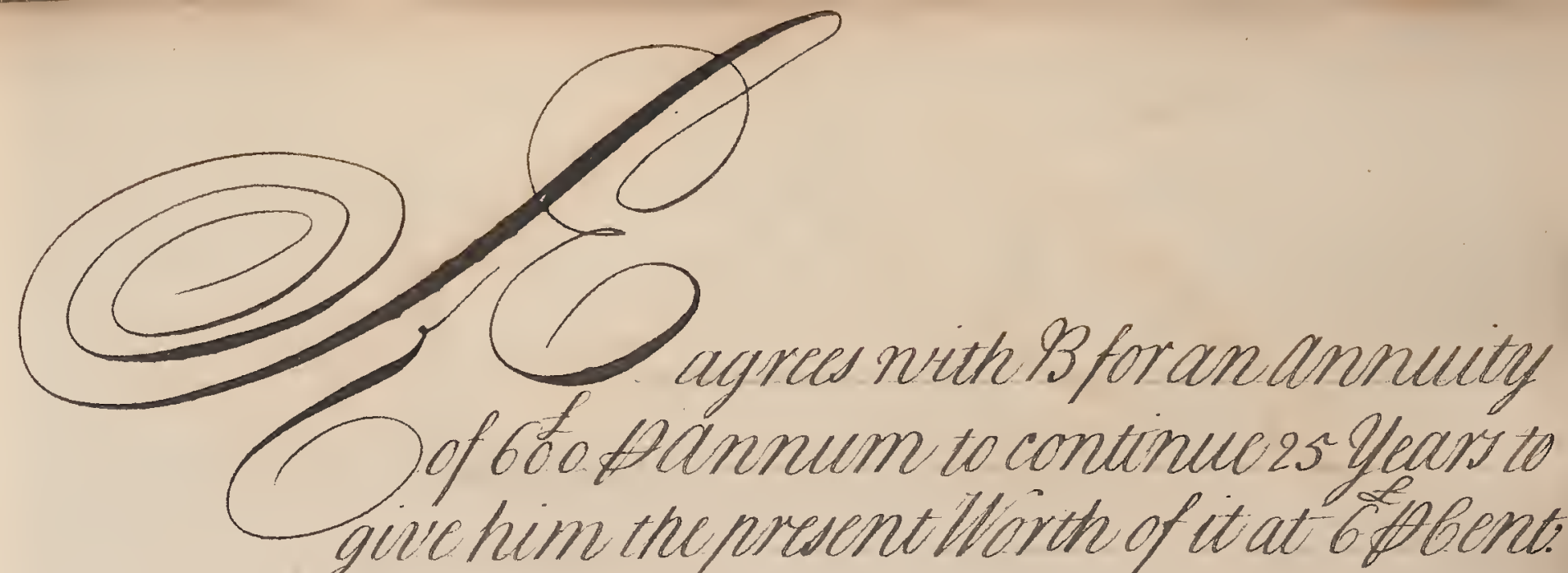
Dconnum, but not having money enough by him offers to make over wo him a Frechedo Estate of 12 f Inn um at the same. Interest, What Money besides will pay his furchase?

$$
\begin{array}{r}
12.70335 \\
600 \\
7670.01000
\end{array}
$$

Ans: $\frac{200.00000}{1470.01000}$ 
<smiles>C#CC</smiles> 

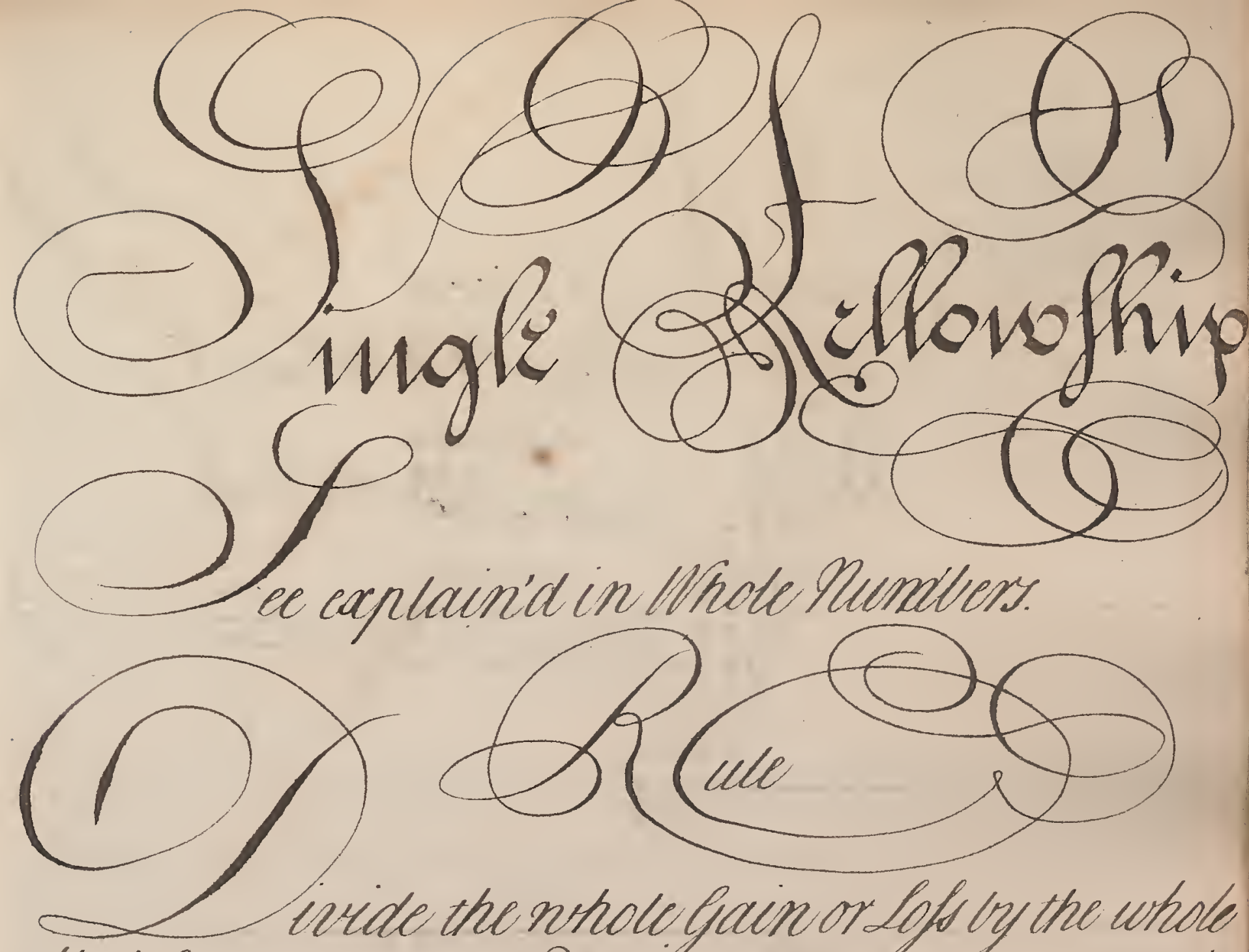

Stock, \& multiply the Quotient by each man's parties= - uvular Stock the several forsducts are the respective fins of each Six or Seven places of Decimals in the Zuoliont are sufficient in most bases.

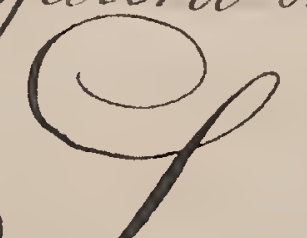

uppofe a B\& Co trading together a puts, sos B700\& 1200 their who ole gain is 336 . What share of it belongs to coach? 
$: \begin{aligned} & \mathcal{L} \\ & 500\end{aligned}$

- B 700

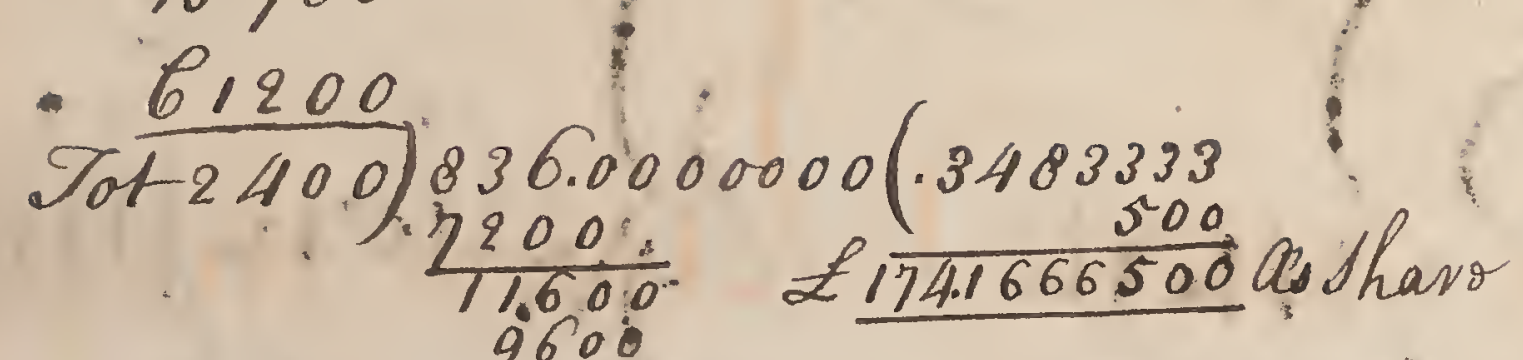

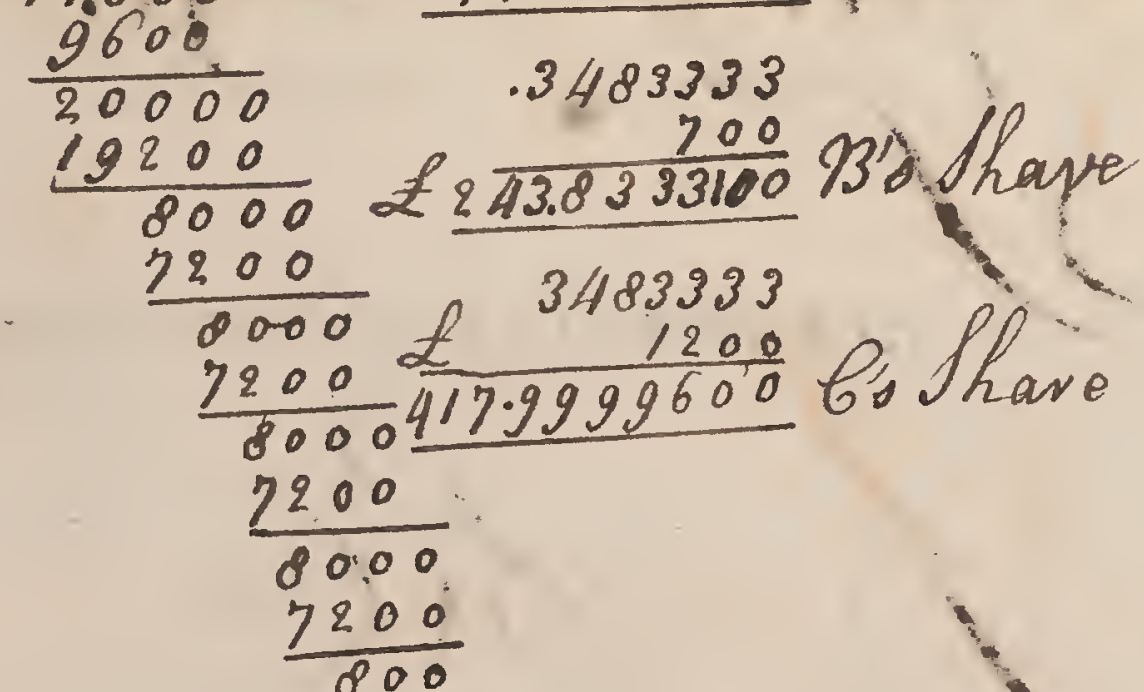
$\frac{7200}{800}$

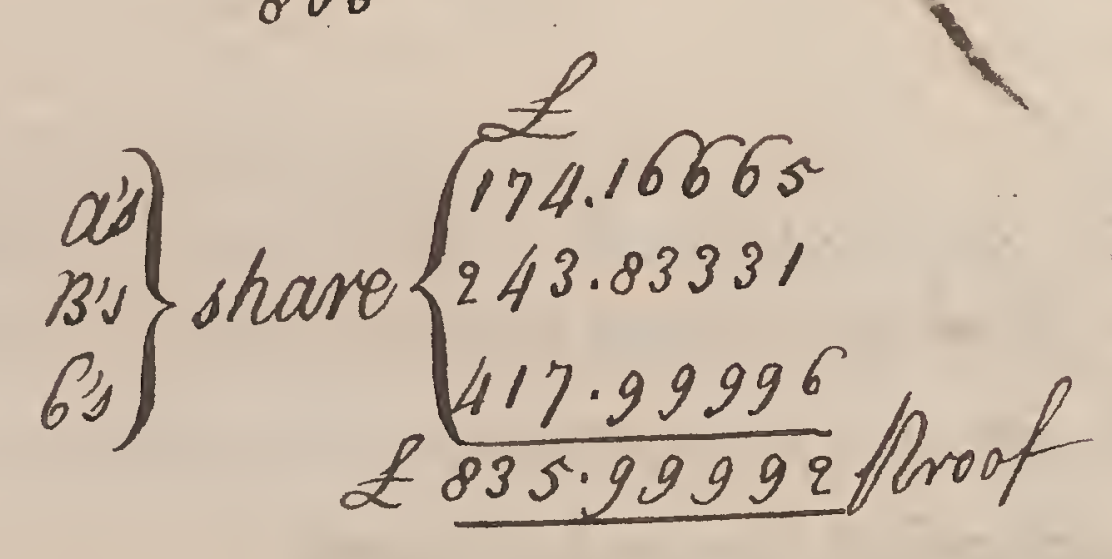




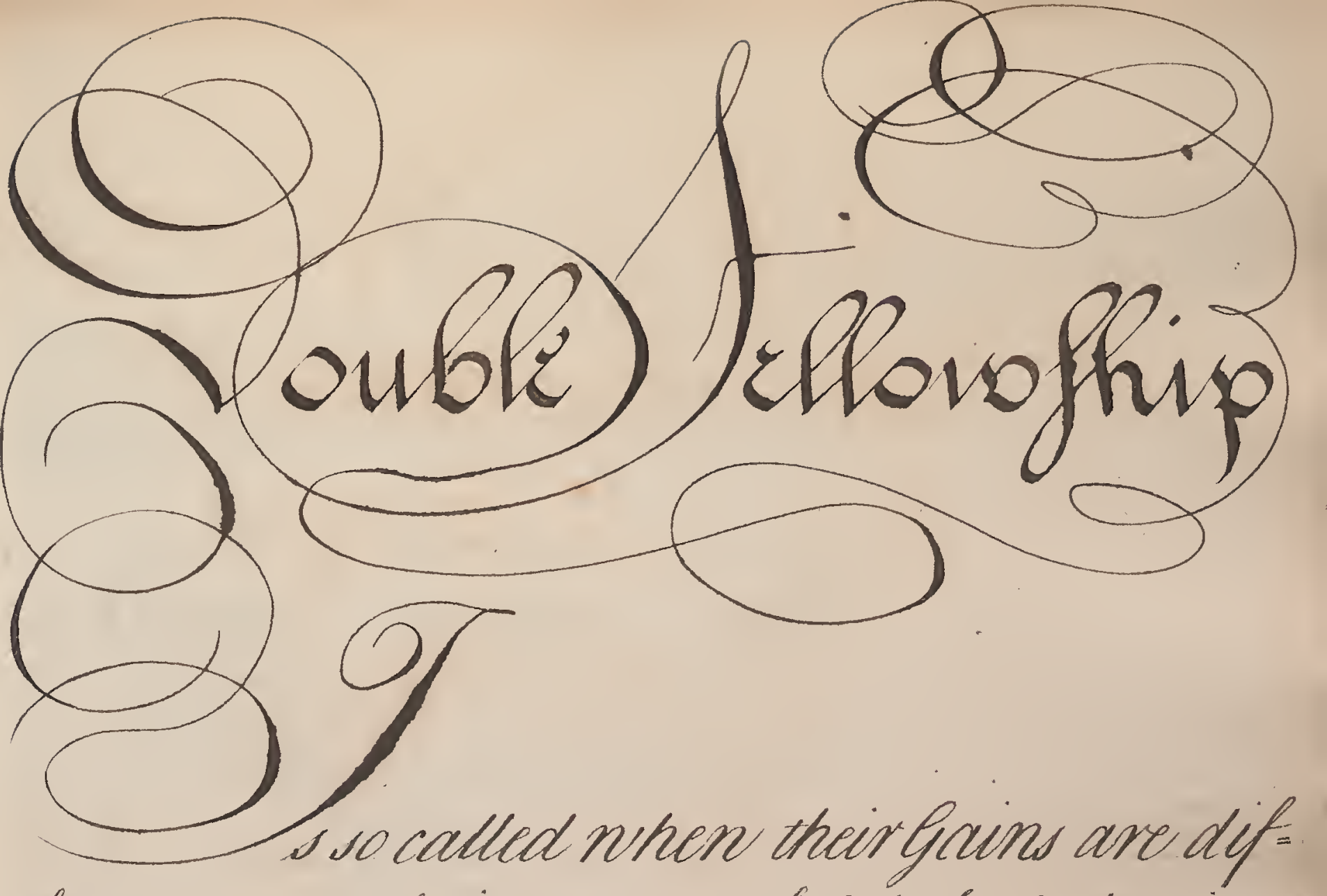

ferent not only in respect of thair fecks luet in re spect of the im of Gontinumece in bompany, Therefore to work the Sums otserve this generwel Rule.

\section{sum of the}

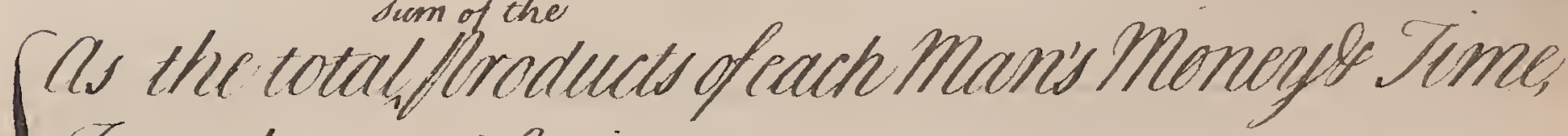
So co the colallfain,

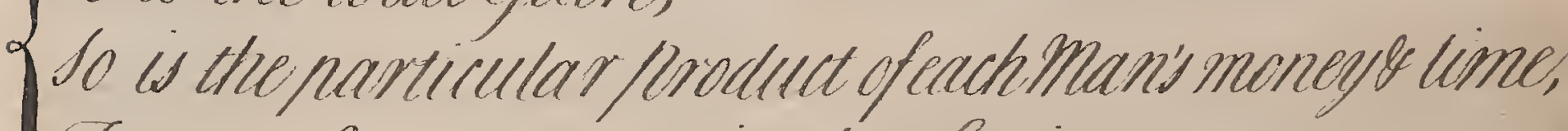
To cach mans partiaular Goin. 



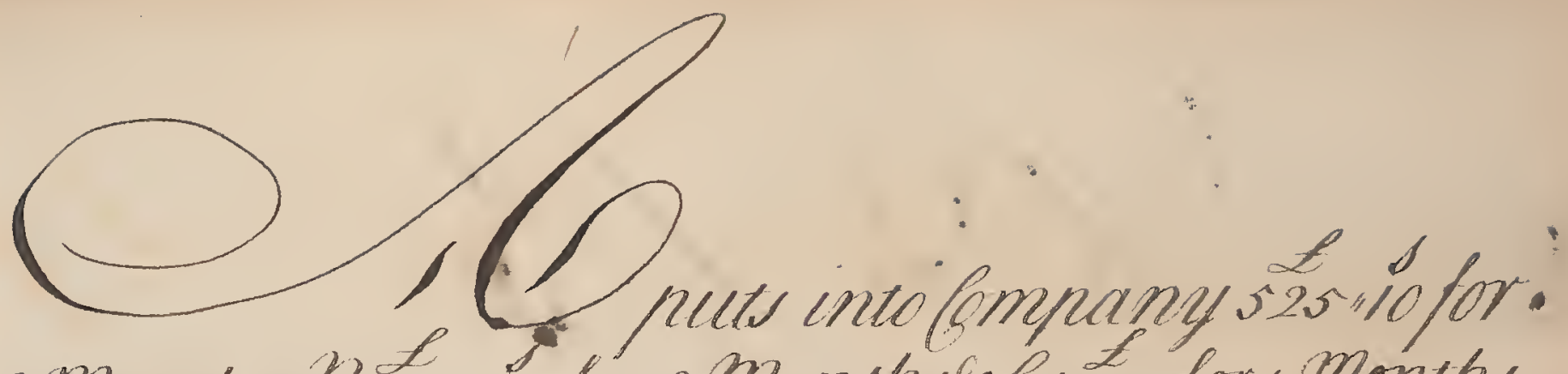
6 Months 13302115 for 8 Months b 1000 for 4 Menths they gained in alli2: 6612. Show muets is that for each?

$$
\begin{array}{ccc}
\frac{L}{1025.5} & 3382.5 \\
\frac{6}{3153.0} & \frac{61000}{306200} & \frac{11000}{3062.0}
\end{array}
$$

$\frac{4000.0}{10215 \%}$

.125952

$\frac{3153}{377856}$

629760

125952

$\frac{377856}{397.126656}$

$$
\begin{aligned}
& 1286.600000(.125952 \\
& 10215 \\
& 26510 \\
& \frac{20430}{60800} \\
& \frac{51075}{97250} \\
& \frac{91935}{53150} \\
& \frac{51075}{20750} \\
& \frac{20430}{320}
\end{aligned}
$$

.125952

$\frac{3062}{251904}$

755712

$\frac{3778560}{385.665094}$
125052

$\frac{4000}{503.808000-6}$ Gain 305.66502493 Gain

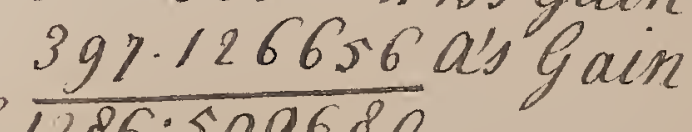




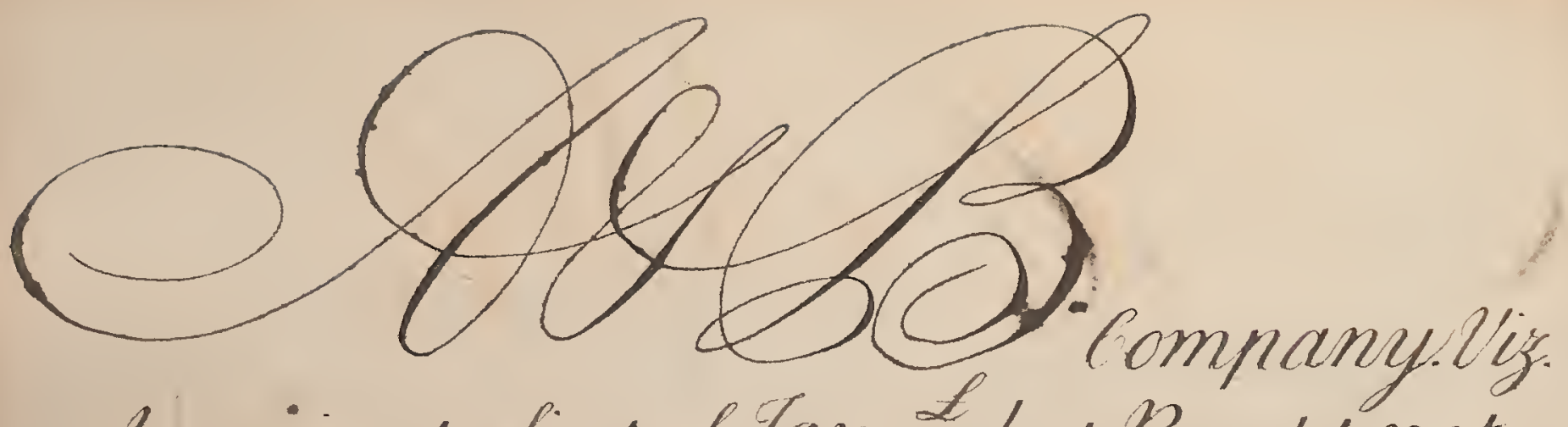

et put in the first of frons to but 3 could not nut any Money in till the r. "f May following. What must si then put in to have an equal. share with a at the Years b nd?

Months $\mathcal{L}$ months

$12: 59:: 8$

$$
\frac{12}{808.0}
$$




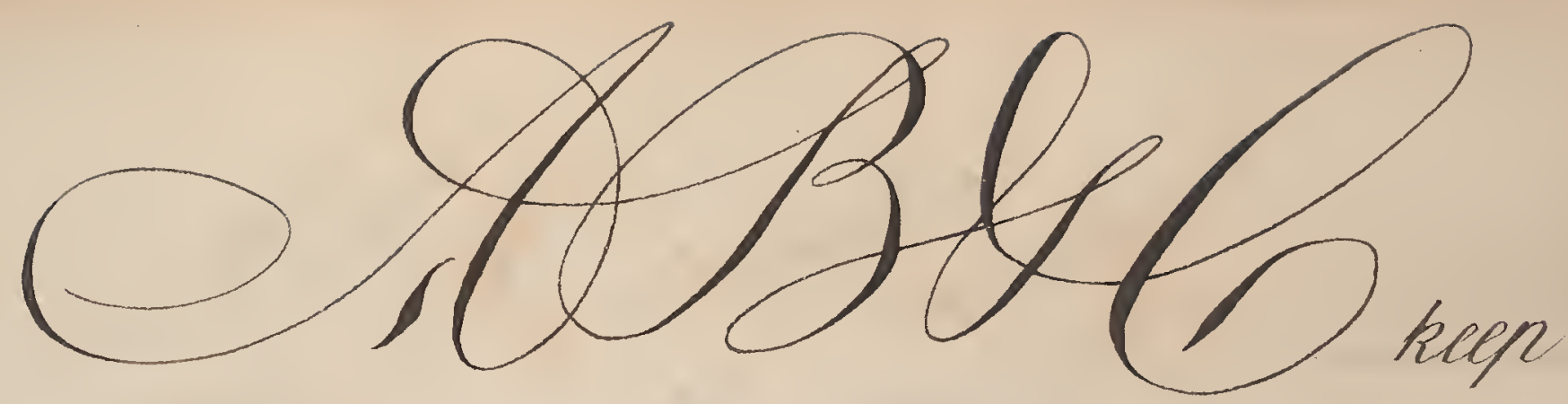

Company; a put in the first of March 6o L B Put

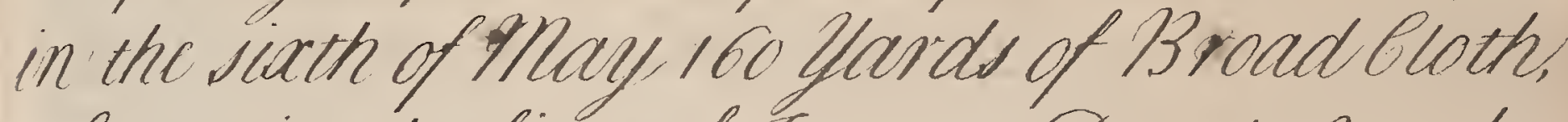
sbuut in the first of June 240Ducats: On the first of fanuary folloning they accounted their Gain: of which A\& B took up 4s6. B\& b took up $43 \%$ b be A took up 375 . The Dustion is what was gain'd as well in the wholo as a-part, whats valued ayard of bloth at, \& what b's Ducats rea. He was?

$$
\begin{aligned}
& \text { L } \\
& 156 \text { ales } \\
& 431386 \\
& \text { 3) b be a } \\
& 2 \longdiv { 1 2 6 2 } \\
& 631 \text { Totalgain } 631 \\
& \begin{array}{l}
431 \text { skb } \\
200 \text { dis Gain }
\end{array} \\
& \begin{array}{l}
\mathcal{L} \\
631 \\
\frac{375}{256} 73 \text { Gain }
\end{array} \\
& \mathscr{L} \\
& 631 \\
& \frac{456}{1756 \text { gain }}
\end{aligned}
$$




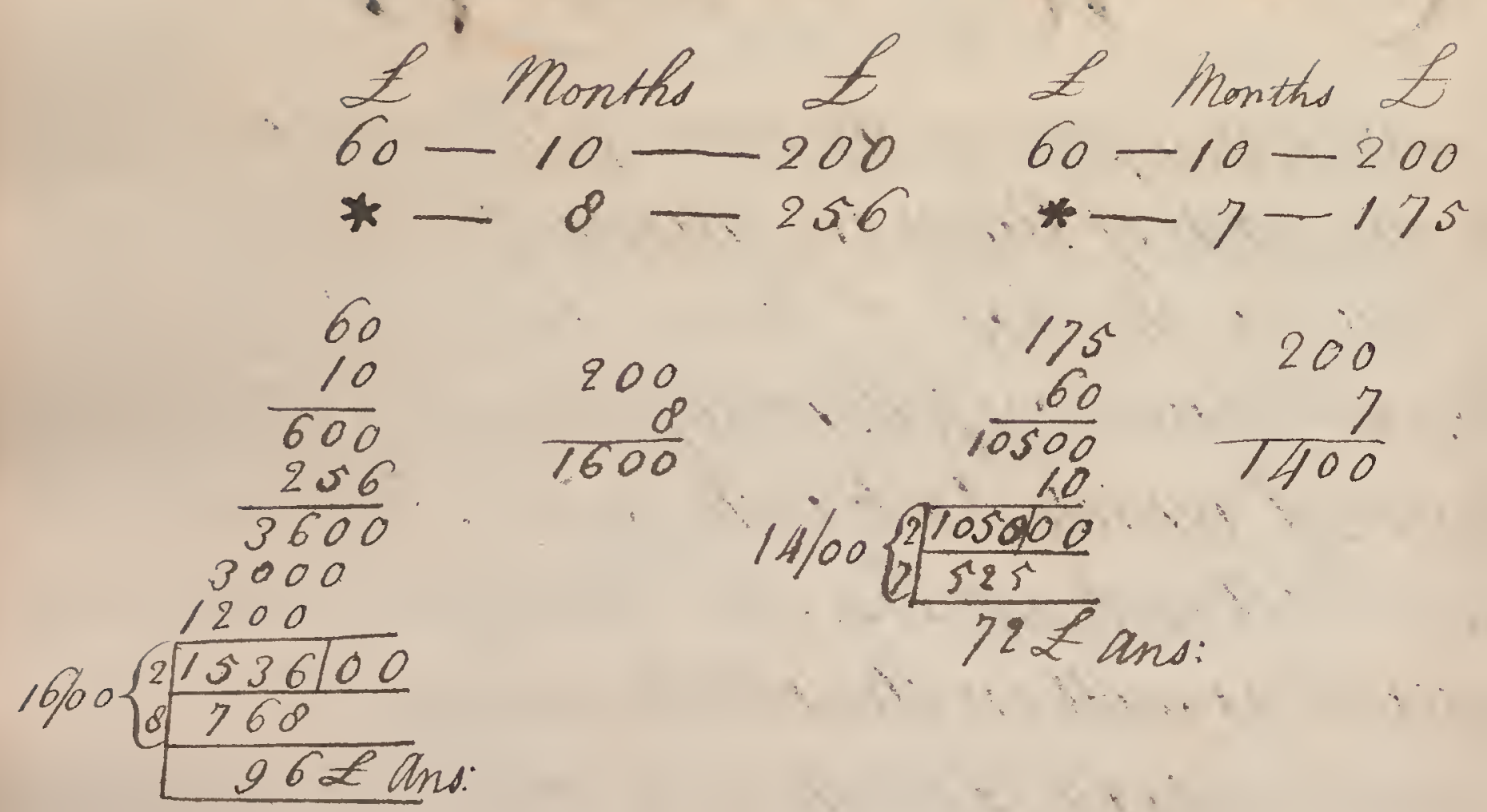

L qo.

$160: 96:: 1$

$16 \%\left\{\begin{array}{l}2 \frac{96.90}{4.8} \\ .6\end{array}\right.$
Ducats Ducat 240:72: 1

$$
240\left\{\frac{27.00}{\frac{3.05}{.3125}}\right.
$$




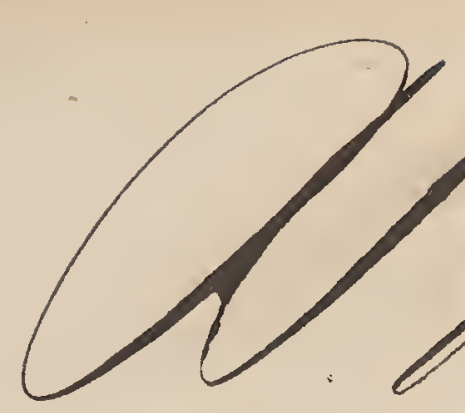

L

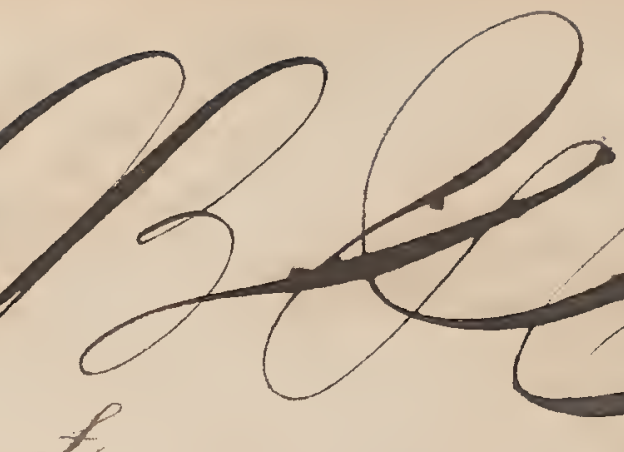

Companyle put in together 3822 O's money vers in 3 Months; Bs mancy wasins Months; sis moncy wasin

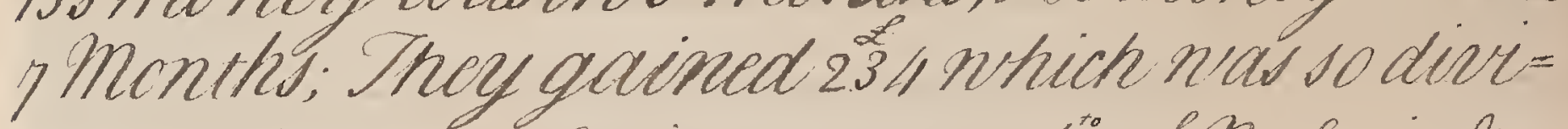
ded as the: of as Gain nes equat:' of Bs lyains: of Bs Gain equal"s of b's Gain What dia each Mler: "chant gains put in?

$$
\begin{aligned}
& \text { sup: } 2 \\
& \left.\begin{array}{cc}
20 & 13 \\
105 & 3 \\
140 & 6
\end{array}\right\} \text { gain } \\
& \mathscr{L}
\end{aligned}
$$

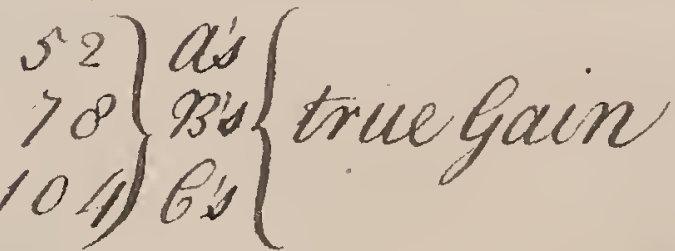

$$
\begin{aligned}
& \mathscr{L} \mathscr{L} \\
& 315: 234:: 70 \\
& 315) \frac{70}{16380022} \\
& \frac{1575}{630} \\
& \frac{f}{52} \frac{3}{156} \\
& \mathscr{L} \\
& \frac{30}{390} \\
& 390 \\
& \frac{728}{1274) 3822(3} \\
& 630
\end{aligned}
$$

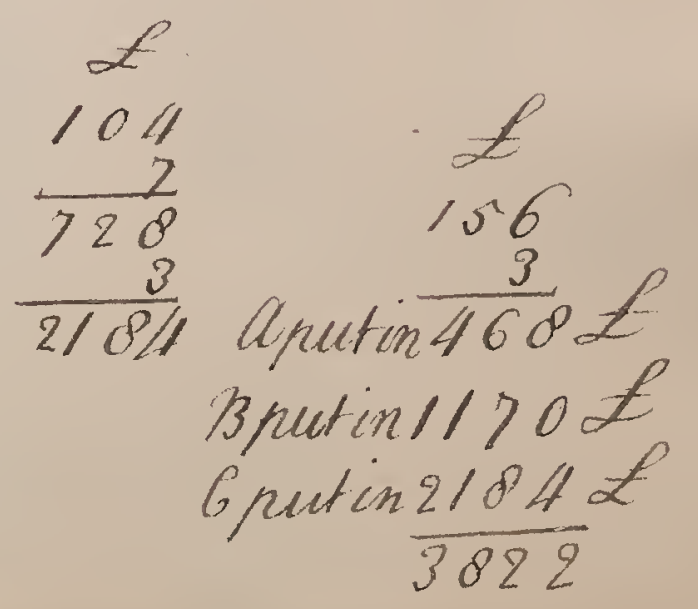


<smiles>CC#CC</smiles> 


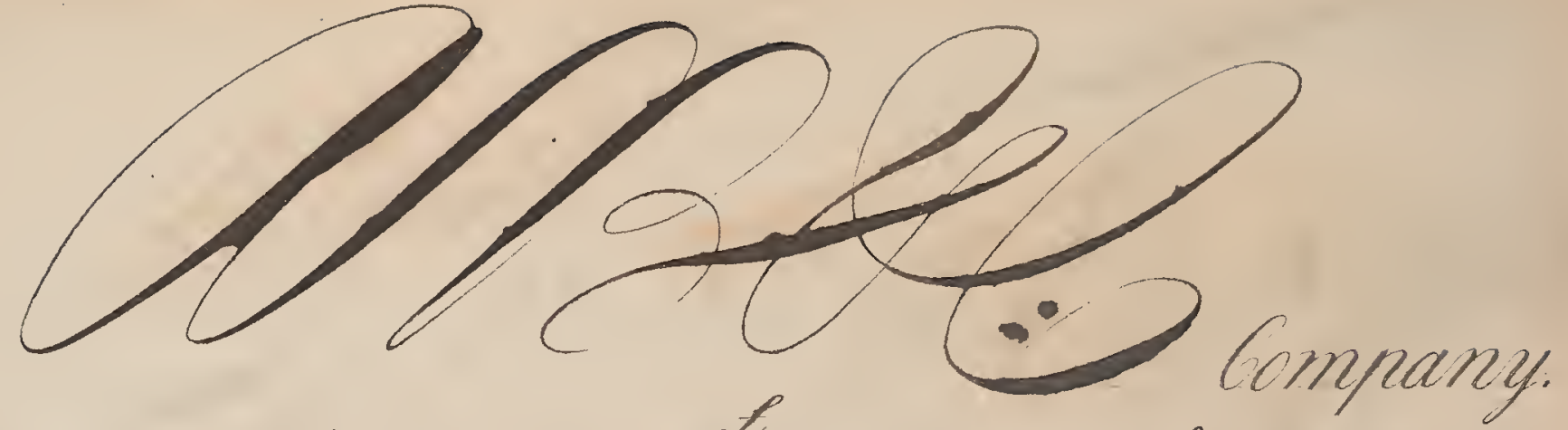

A put in their Sanuary-too \& the first of may puts in 150 mores on the first of september takes out 30 the Remainder stays in till the Year's b nd. Spur in the first of January 250 \& on the first of June so mores on the first of november:soo more which continue in till the Year' b nd. b jut in the first of Ian: 300 \& on the first of April lakes out 2008 on the first of August takes out so more the Remainder stays in till the Year's bod: What must each have of the gain which nos 133 ?

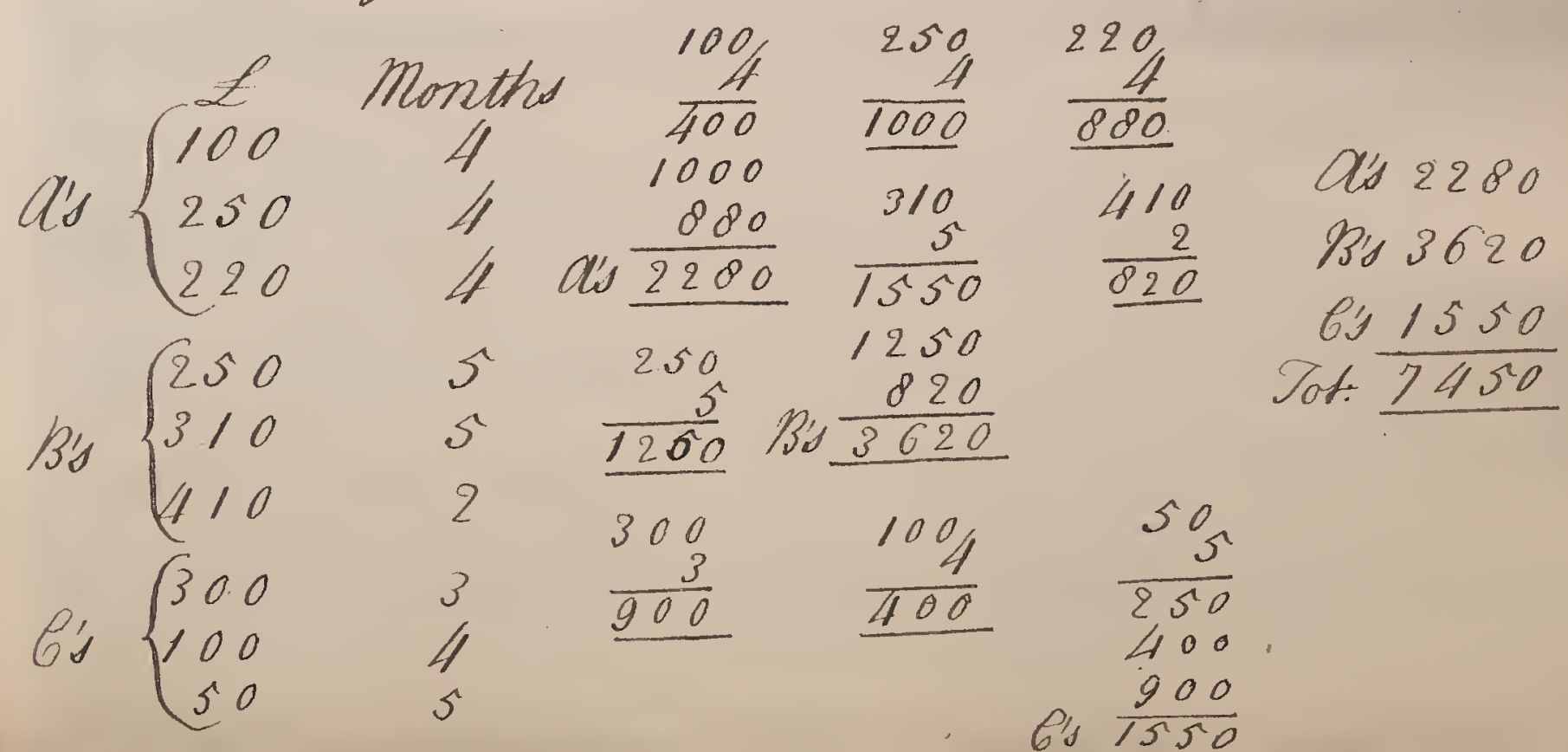




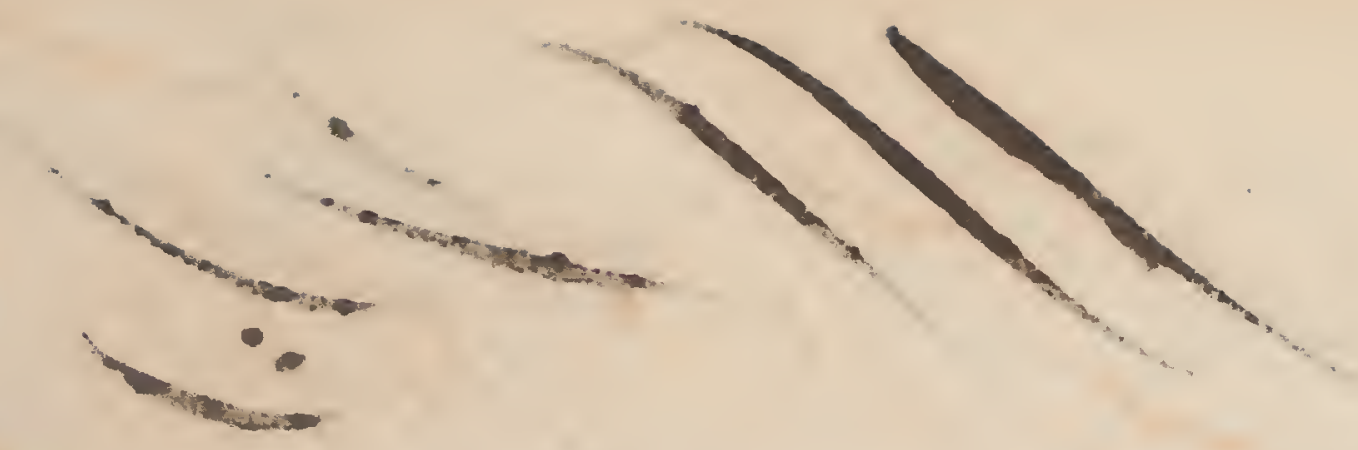

7450

$$
\begin{aligned}
& \frac{150}{1500} \\
& 58500 \\
& 52150 \\
& \frac{63500}{59600} \\
& \frac{5.9000}{37250} \\
& \frac{32500}{14900} \\
& \frac{14600}{2600}
\end{aligned}
$$

$$
\begin{array}{r}
.017952 \\
1550 \\
\hline 092600
\end{array}
$$

89260

$$
\left.\begin{array}{l}
\frac{17852}{27.670600} \text { As } \\
64.62424031 \\
40.7025606 \mathrm{~s}
\end{array}\right\} \text { gain }
$$

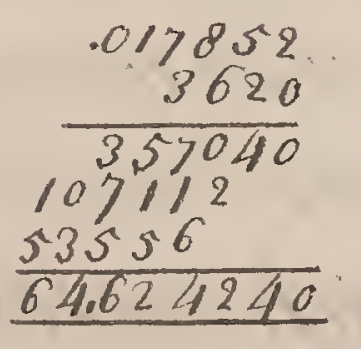

$$
\begin{array}{r}
.017852 \\
3620 \\
\hline 357040
\end{array}
$$$$
107112
$$$$
\frac{53556}{64.624240}
$$

.017852

2280

$\longdiv { 1 4 2 8 1 6 0 }$

35704

35704

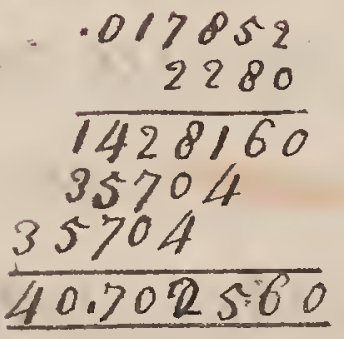




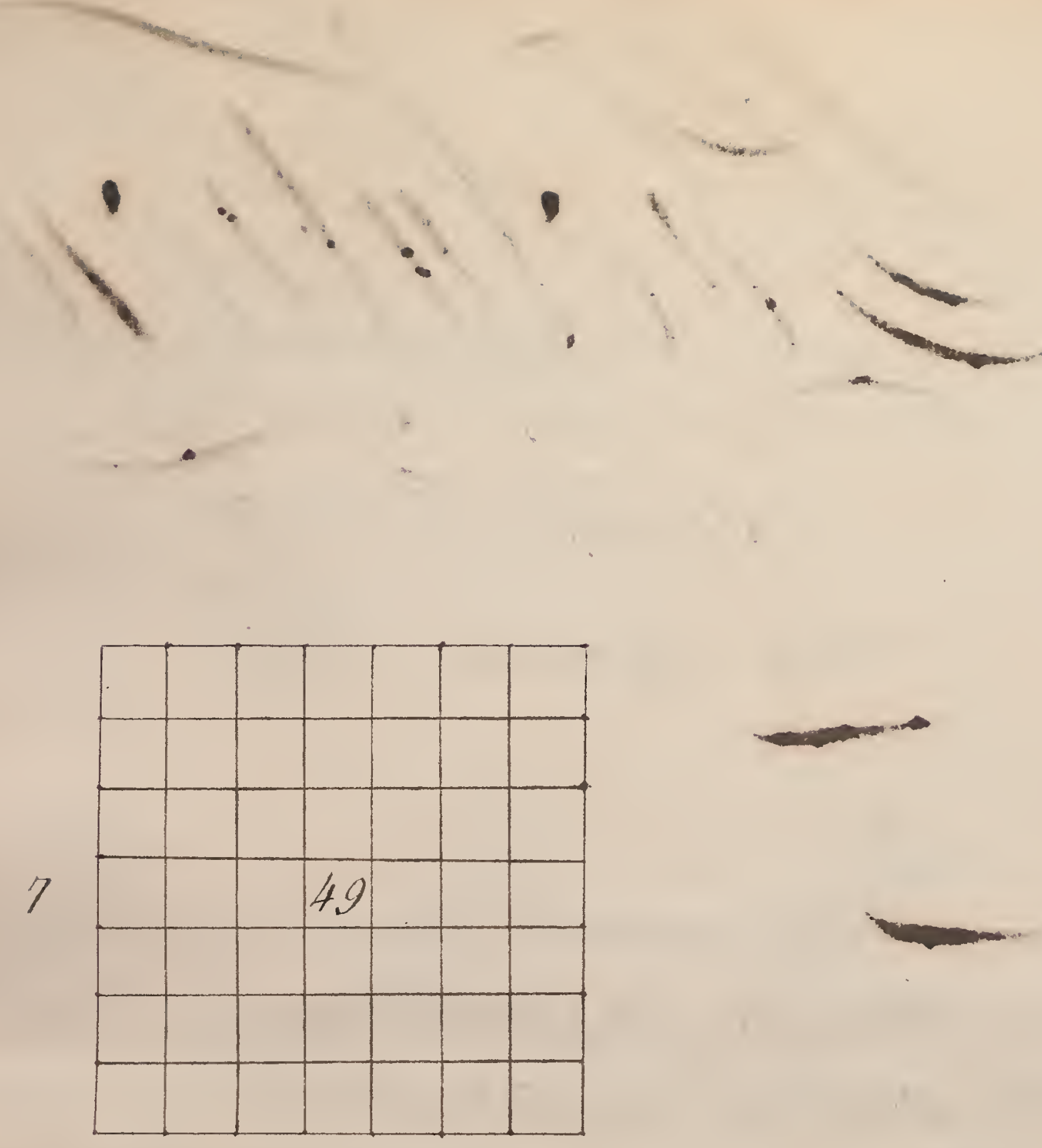


Square Membless an cither Single orfomnowne.

a Single Square Humber is alm ayys lefs than 100 \& its hoot is found at once by. The foiloniong Sas. Hte.

\begin{tabular}{|c|c|c|c|c|c|c|c|c|c|}
\hline 1 & 4 & 9 & 16 & 25 & 36 & 49 & 64 & 81 & Square \\
\hline 1 & 2 & 3 & $H$ & 5 & 6 & 7 & 8 & 9 & Roots \\
\hline
\end{tabular}

Supnofe gb a Square Rumbir given nihose Root is required look in the Jable for the neat least squane numbern'hich is \&1 whose Root isgs the kemain derisls.

A Gompound Square Alumber is made vy the Mul= tinlication of a cronore figures by themselves \& nlioans 100 as 132 which is 1/ times 12; or 69 which is 1stimess? To find the Root of any compaind Square Humber as sliphese $270 \%$. 
first you muse destinguiste it into single Squares by pla=

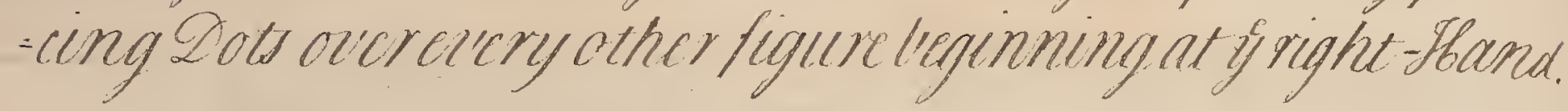

$$
\text { Thus } 2 \text { icia }
$$

And so many Dots as hrepuen so many fllaces will the Root consist of

Secondly draning a crooked fine on the right bund of your Bumber as in Division find the koot your first Singli squares nucisin the Wutient.

$$
\text { visz } 20 \dot{4}(a
$$

Thirdly placing the Square of the Root found under thefirst single Square surtracts setdonn the Remainder tringing downt to it the next single Syuares call thes line a Resolvond.

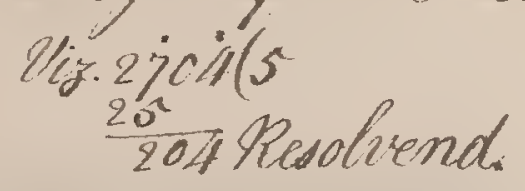

Sourthly draming another crocked Line on the left hand. of the scrid Resolvend, place buyond it the doulle of the Que. - tient, in the Maniner ef a Divisor.

$$
\begin{aligned}
& \text { lig. } 2 \dot{2}_{25} \dot{4}(5 \\
& \text { 10) } 204 \text { Resoliend }
\end{aligned}
$$

Fifthly diriding the Resolvend all but the Init's place, ty the said Divisor set don't the number of times it goss both in the 2wo. - ients on the right hand of the Divisor dis 2io ils

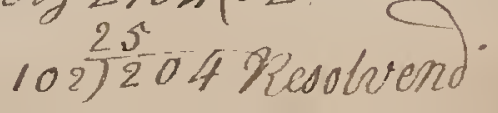


Swarthy mullinlyeng the whole D wis or II the Figure last pad in the Zuetiont set don m the forded un-der subtract it from the Resolvent. V'sisoits2

$10.25 \frac{25}{204}$ Resolvent

$\frac{204}{0}$

The fame Question lineally complained.

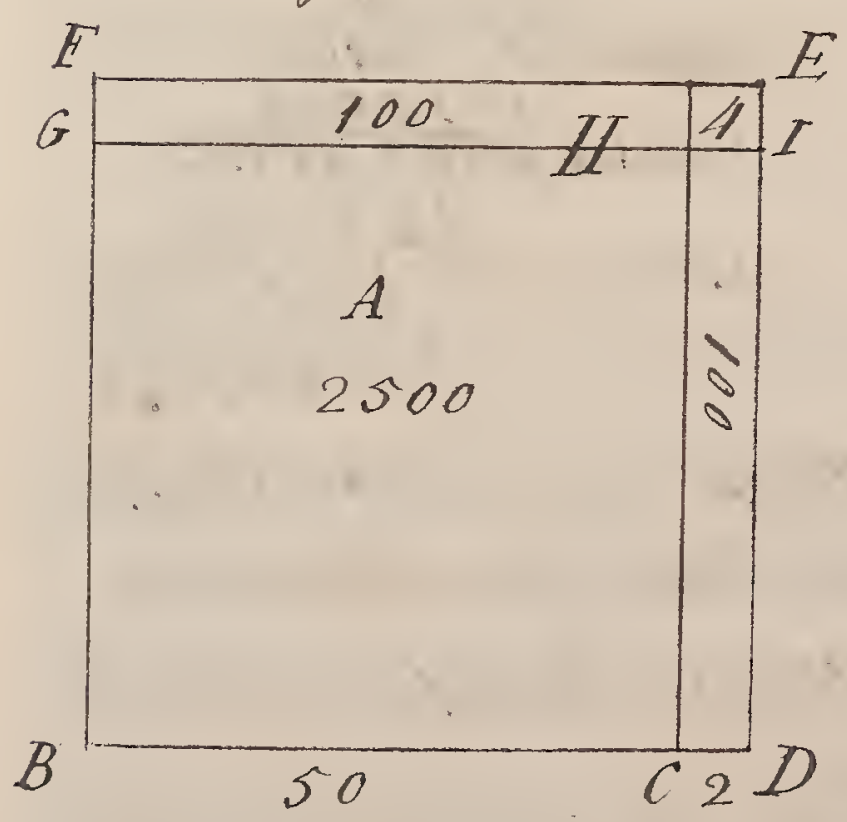

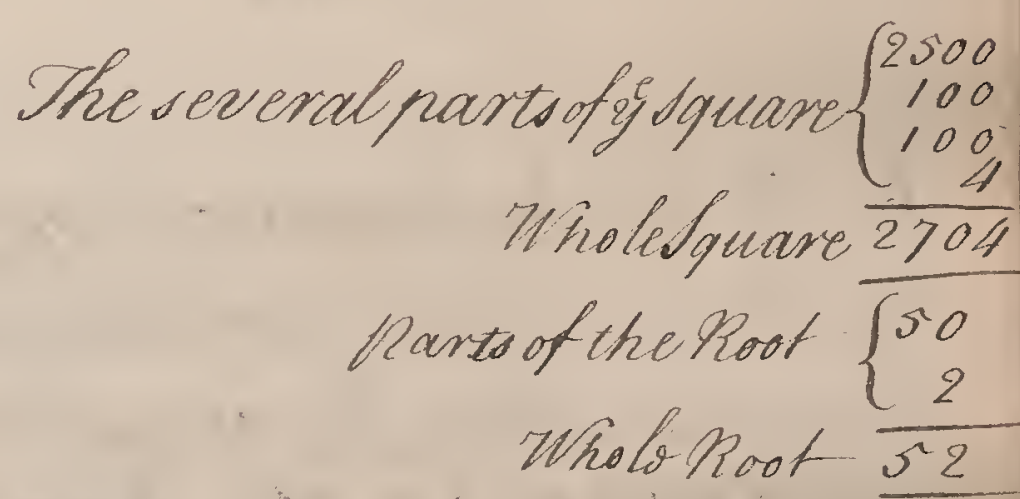

Whats the Square Rood Whats the Square Root of 119716 ?

of 21410384 ?

$$
\begin{aligned}
& 119716(346 \\
& \frac{9}{297} \\
& 645 \\
& 6 8 6 \longdiv { 2 1 1 6 } \\
& 1116
\end{aligned}
$$

$$
\begin{aligned}
& 214183814(4628 \\
& 16 \\
& 86) 541 \\
& 9276 \\
& 92983 \\
& 9248973984 \\
& 13984
\end{aligned}
$$




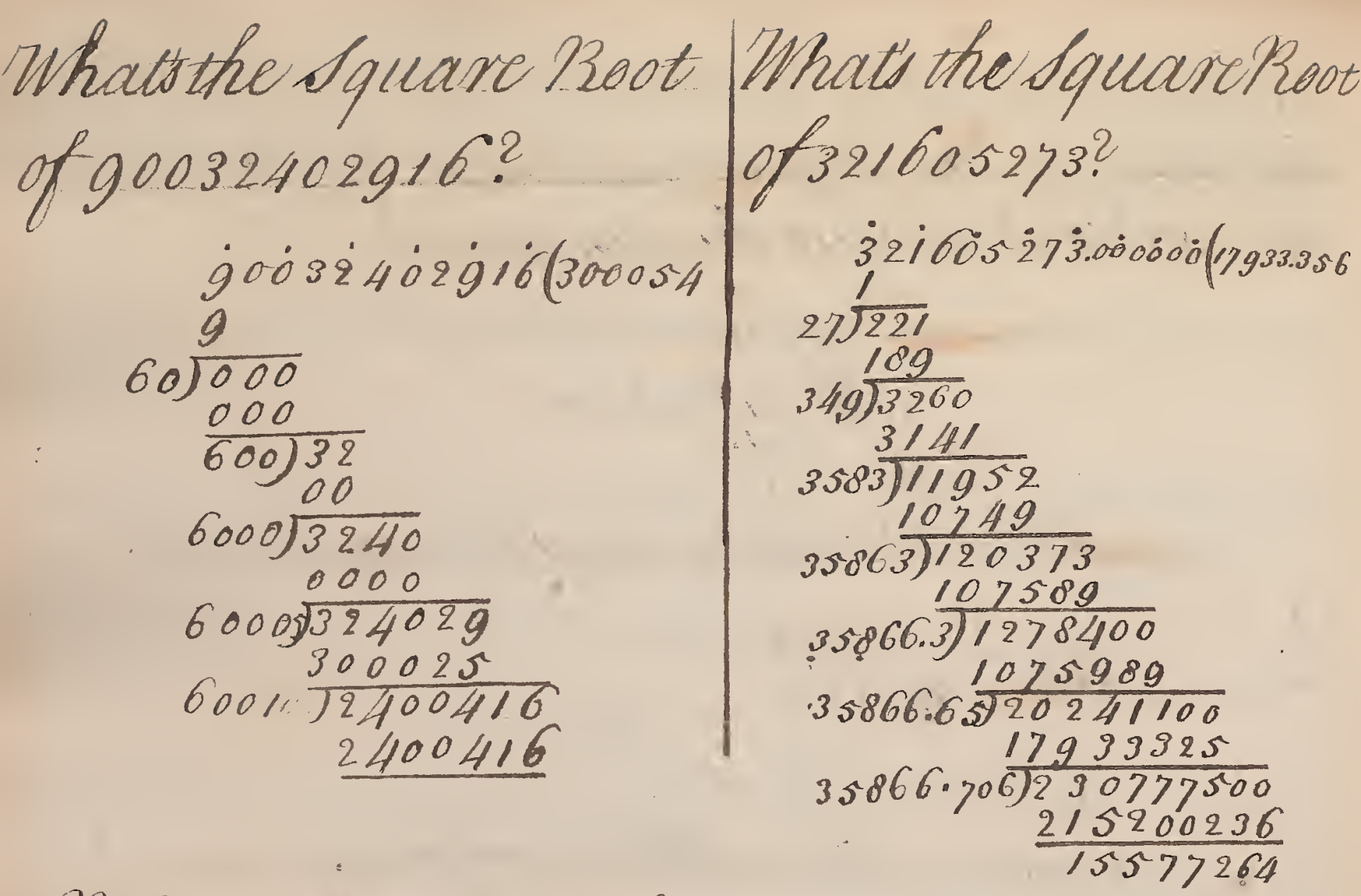

N. B. If the Product happens to be more than the Resolved you may be sure you have ta: hen it a time too much onus therefore set a Refs Figure in the Root.

Secondly the Operation for finding a new Resolvent se Divisor mut be repeated for every Figure placed in the ho ot except the first. If you would Extract the Square Root of a Vul- gar Fraction Extract the Square of the hume. - rater the Root the rcofnipuce for a numerator then Extract ye square of the Denominator the 
Root of which place for a Denominator. But if the Fraction happens to be surd that is compos of such numbers nits ese Root cannot be exact - ty found: Reduce it to a Decimals the observe

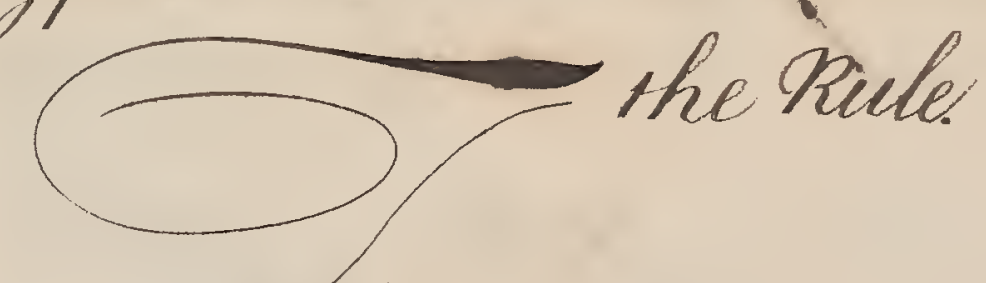

o extract the Square Root of a veii= mat Fraction.

( he Decimal must be nide to consist of an even number of faces then wisents spervateas before. Note firs if you would find the frastionallfarts of any Rem. anmexpairs of byphersep rowed w farasyow please, secondly if a mixed number le given the be=cimals must be coven.

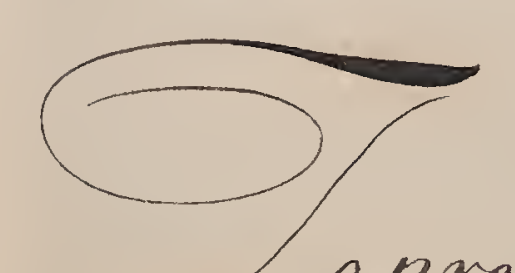

o prove Extractions of theidquare Root.

add in the remainly the Root by it sade to the product add in the remainder if array) which if right will le the same with the given Number. 


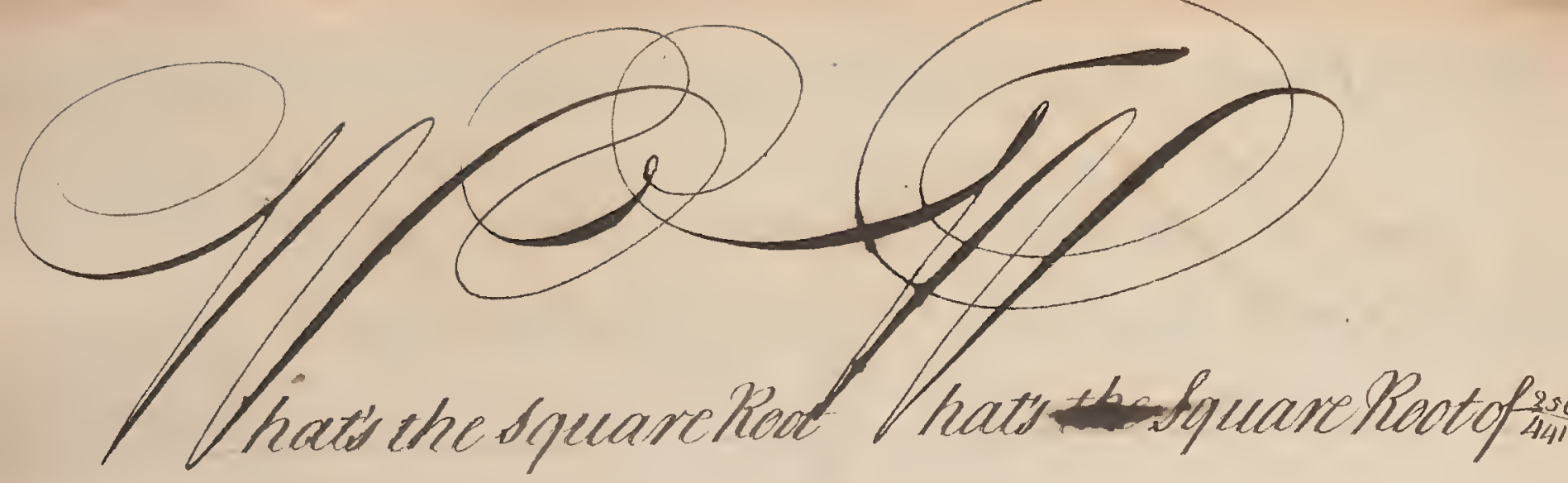
of $\frac{16}{19}$ ?

ans: $\frac{4}{7}$

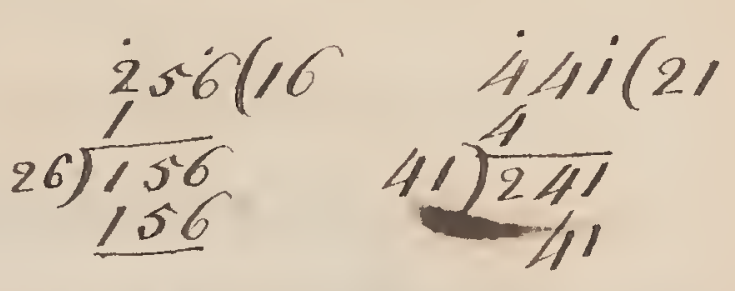

- And:-6.

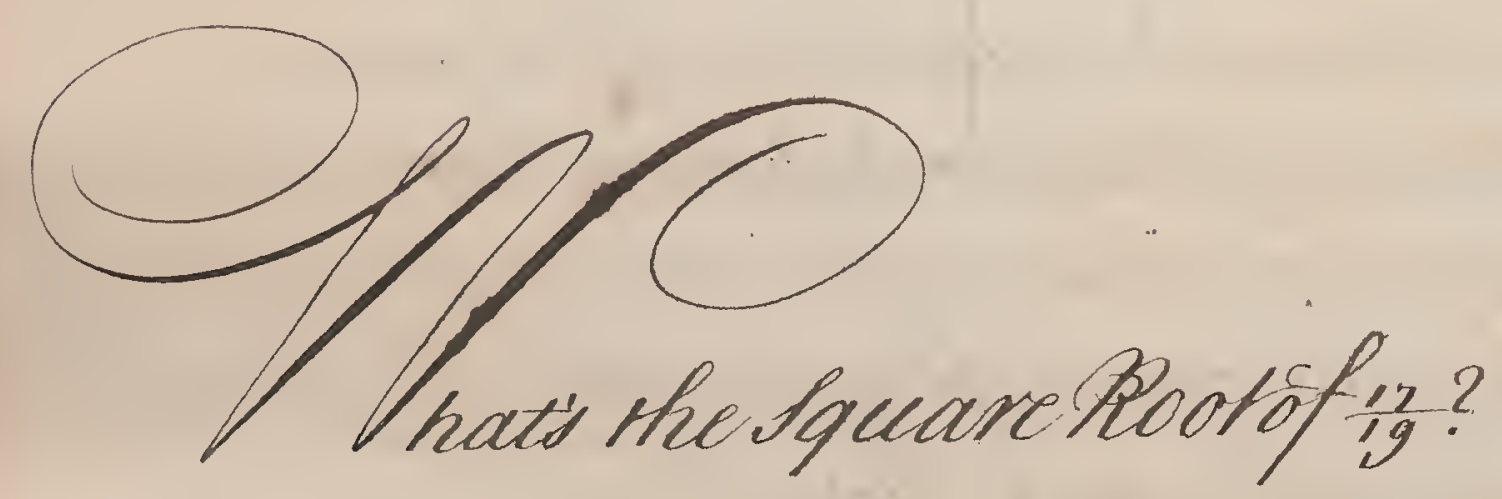

19) 1).00000000(.894)3604 (9459 ans:

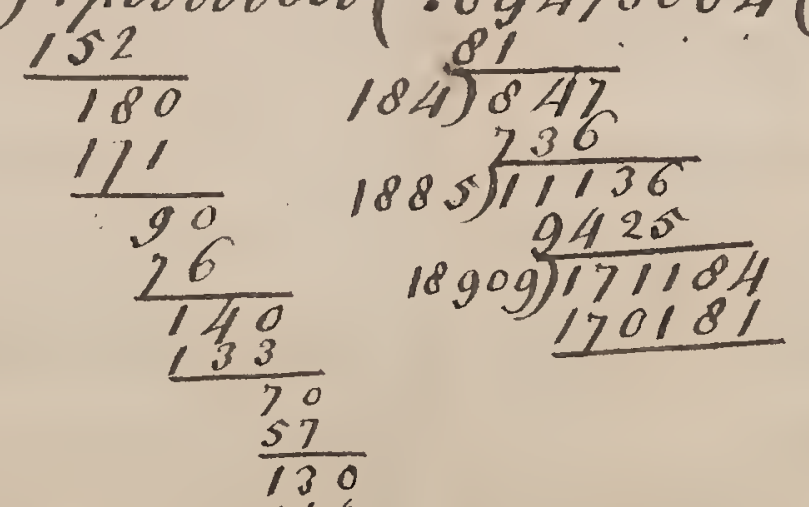




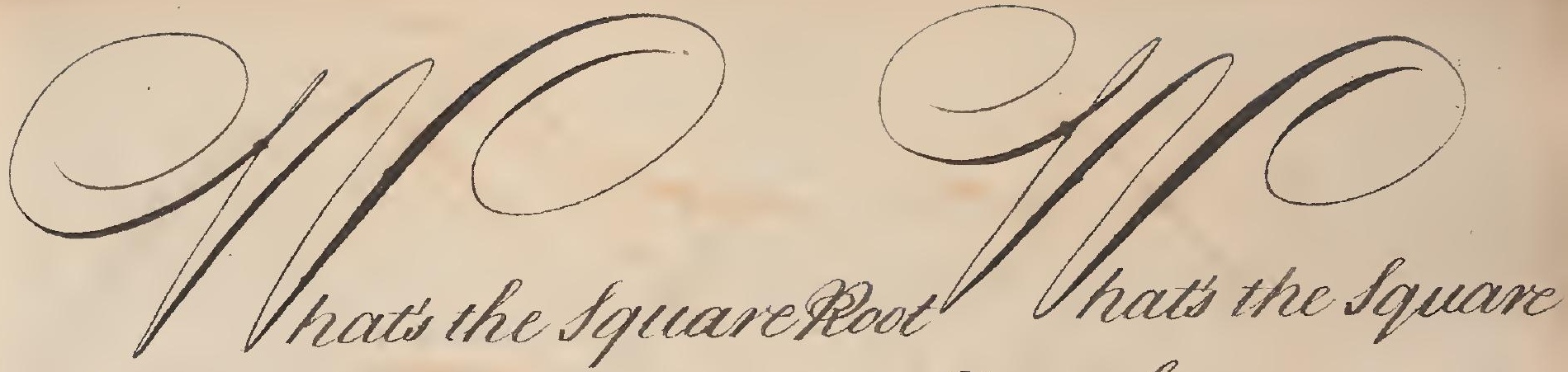

of 31614130 ?

Root of 3513 ?

3i6illi30.000000000(5622.64413

$\frac{25}{106) 668}$

$11 \frac{636}{2 \sqrt{2511}}$

$1 1 2 4 2 \longdiv { 2 9 7 3 0 }$

$1 1 2 4 4 6 \longdiv { 7 4 2 4 1 6 0 0 }$

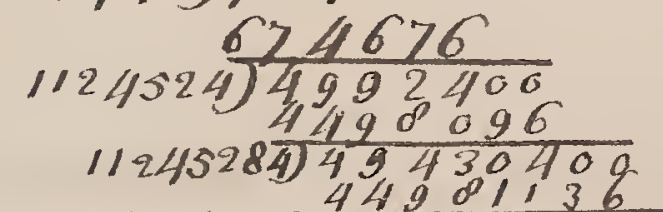

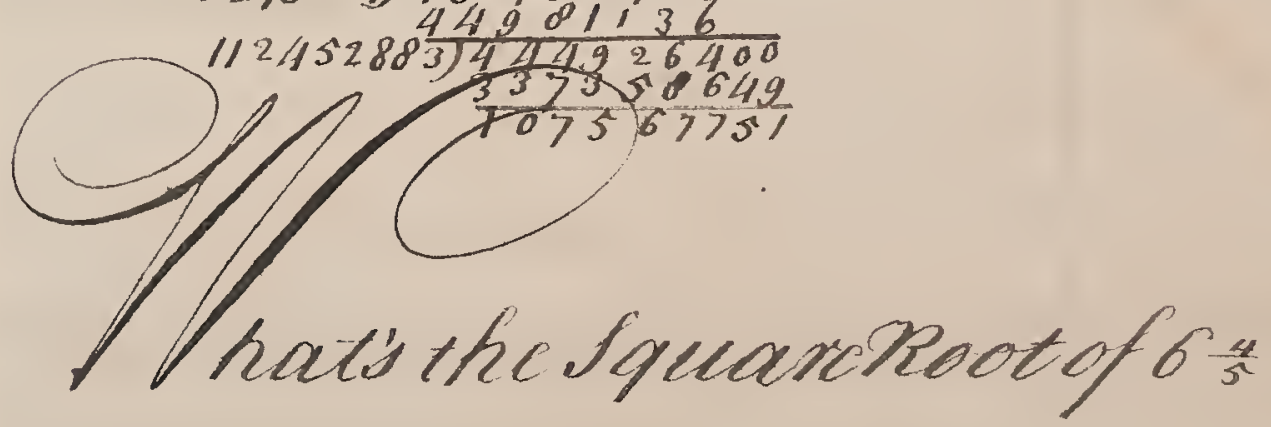

$3 s i / 3.00000000(39.2) 05$

$\operatorname { l o g } \longdiv { \frac { 2 5 } { 1 0 1 3 } }$

$1 / 8 2 \longdiv { 3 2 0 0 }$

$1 1 8 4 7 \longdiv { 8 3 6 0 0 }$

$1 1 8 5 4 0 \longdiv { 8 2 9 2 9 }$

$1 1 8 5 4 0 5 \longdiv { 6 7 1 0 0 0 0 }$

$\frac{5927025}{722975}$

$6.000000000(2.60) 6$ lens:

46) $\frac{4}{200}$

$\frac{276}{2 0 \longdiv { 4 0 0 }}$

$520) 4000$

$5207) 40000$

36449
$52(46) 355100$
312876
42224 



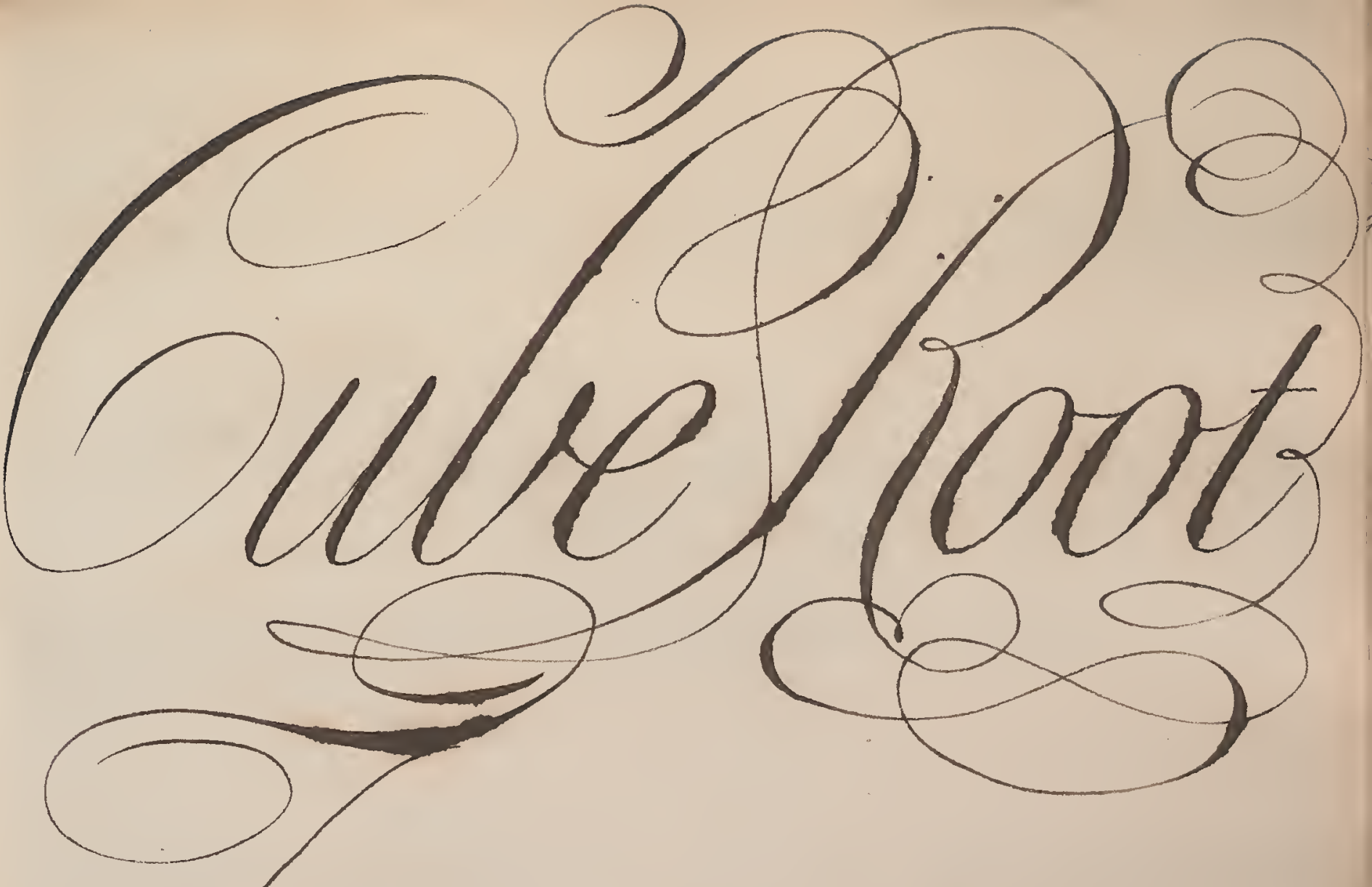

Lhe bxeraction nithereof consists in find. ing the side of a Solid Stigure wi hose Song th ts readeh \& Depth are equal, or puimerically speaking it consists infinding out nhat number multiplicd onice into it's self nill produce the numbersthes the butre Rootof 343 is 7, for $7 x y=49$ \& $49 x y=33$, the given fube Rootogiven

What a fibe is may be seen by the folloning Fiqure. 

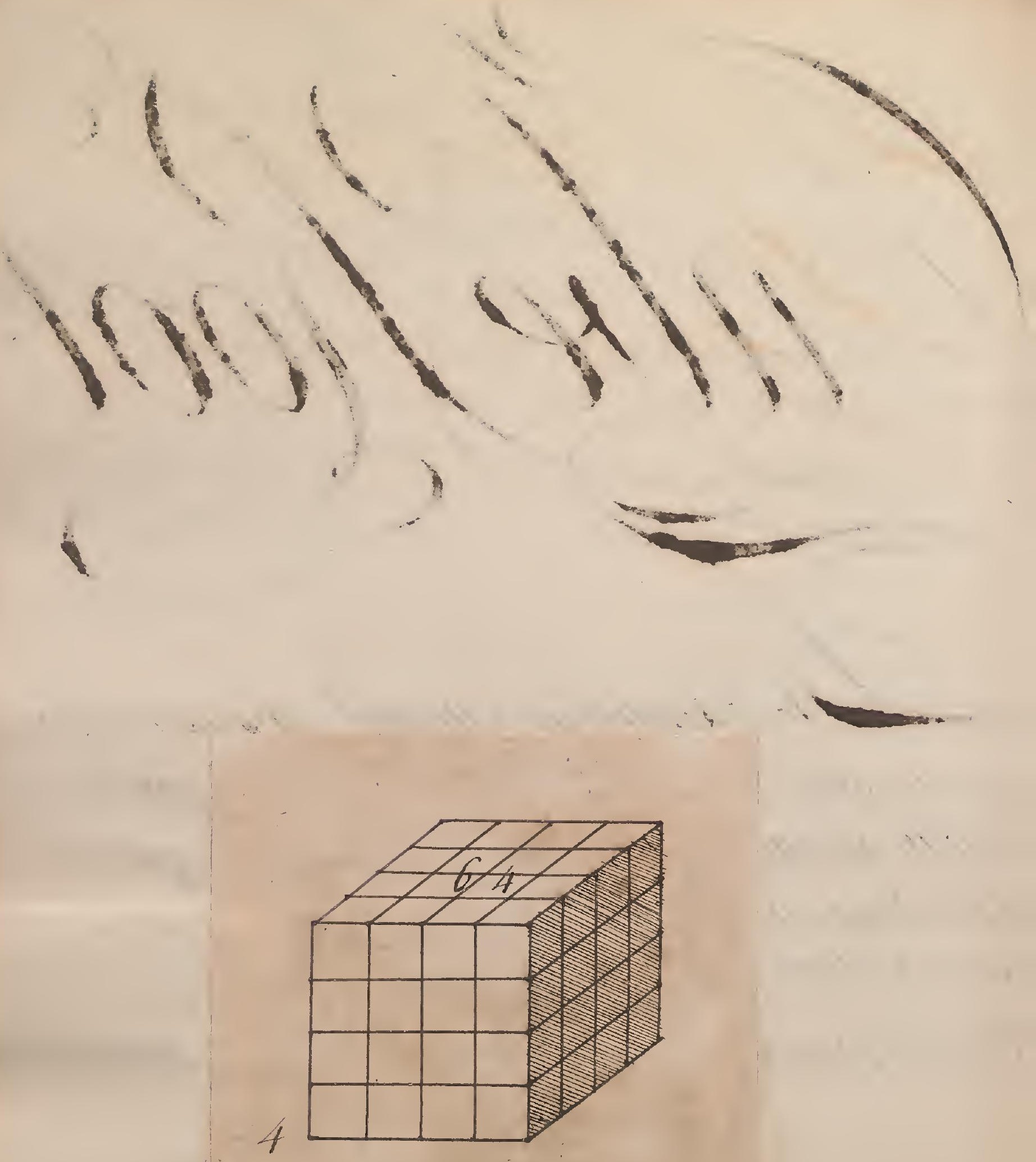

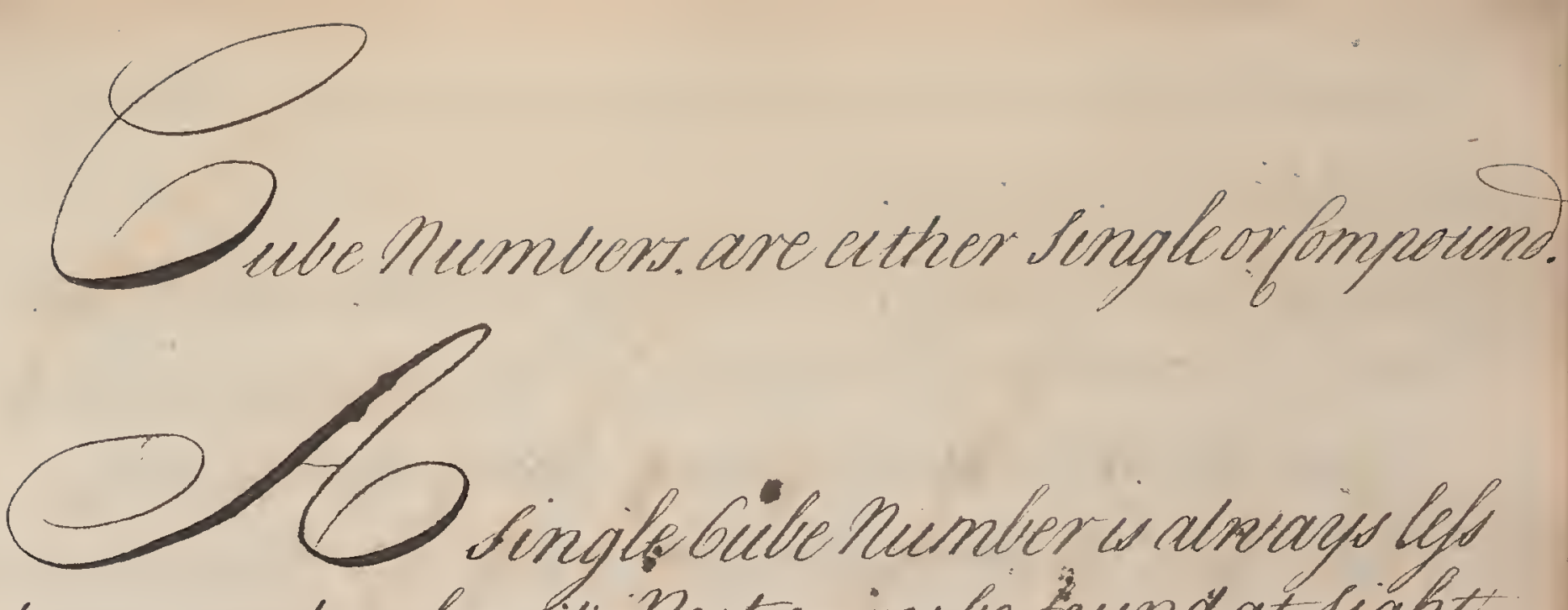
than 1000 therefore its Root may be found at sight by the following Sablesforif yow involve b to the $3^{\text {" }}$ howerits $2168 \mathrm{c}$.

\begin{tabular}{l|c|c|c|c|c|c|c|c|c|} 
Roots & 1 & 2 & 3 & 4 & 5 & 6 & 7 & 8 & 9 \\
\cline { 2 - 10 } & 1 & 4 & 9 & 16 & 25 & 36 & 49 & 64 & 81 \\
\cline { 2 - 9 } & 1 & 8 & 27 & 64 & 125 & 216 & 343 & 512 & 729 \\
\hline
\end{tabular}

A Compound Bute Number is always more thane 1000 \& therefore the Root ban not be found at first singh us in single bubs Numbers br t mutt be proceeded with as follows.

Suppofe is 625 which is a Compound Number to find the Root thereof.

You must " distinguish it into single babies which is done by placing Dots over cverythird figure beginning from the Right Stand. thus 
And so many Dots as happen so many Places mil the Root consist of.

Secondly drawing a crooked fine on the light Stand of your M umber as in Division set donn as a Lucent the Root of your first sing te Lube.

Thirdly placing the bute of the prot found un: -der the first single bubs, Subtract, \& to the Renin. der bring don m the next single buber which tau a Resowed. Viz. $\frac{\operatorname{sis}_{0} 625(2}{7625}$ Resolved

Fourthly dram a Line under the Resolvent \& tripling the Square of the Root set the said triples square under the Resolvent; so that le - nits in the said triple Square may stand un -der the place of hundreds in the Resolved.

Viz. 1 is 2512

7625 Resolved
12 vriplesquare of 2

Fifthly subscribe also the the triple of the Root so that Units in this may stand under the face of tens in the Resolvend.Vis. signs 2

$\frac{7625 \text { Resolvent }}{12 \text { tripledquats }}$

triple quid 
Sixthly the triple square of the Roots triple root being placed as directed draw a fine render them add them together in the Order they are placed; the Sum is a Divisor. Dis isionit2

$\frac{7625}{12}$ tringobent

126 trine square of

$\frac{6}{126}$ Divisor.

seventhly Accounting all the Resolvendfexcent the place of Units) a Dividend sect now of th tic Divisor is contained in it \& place the Number of times in the Quotient. Viz. 15625 (25

$$
\frac{8}{1625} \text { trinlesolventere }
$$

12 triples square of:

126 Divisor

lightly drawn a fine under chobivisor and milliply the triple square on y the figure last protect placed in the 2 client set the fro -duct so under the said triple square that ll

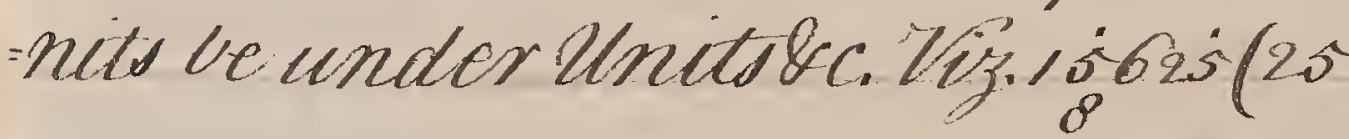

$$
\begin{aligned}
& \frac{8}{7625} \text { niesolient } \\
& \frac{6 \text { triple square of } 2}{6 \text { triple of the hoot } 2} \\
& \frac{126}{60} \text { Divisor }
\end{aligned}
$$

Monthly Squaring thefigurclast placed in the Quotient that tens ins this Multiply its 
Square by the triple Roots place the product so that elvis in this may stand Underlines in the said triple Number. Ais.

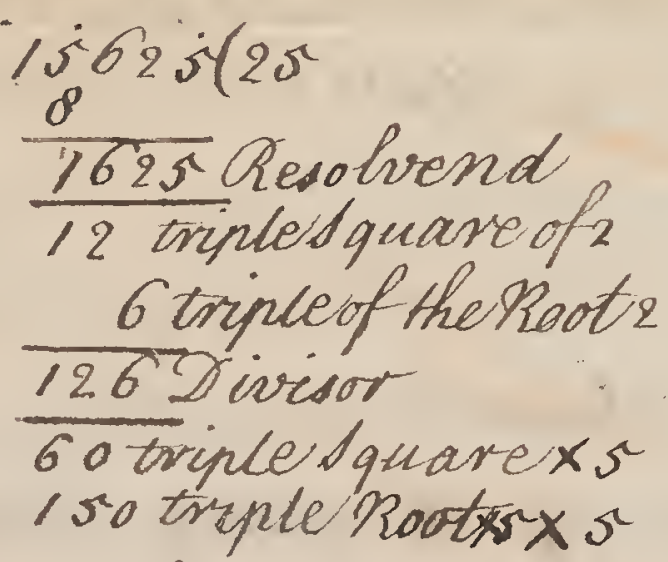

Tenthly subside the bub of the figure last placed in the Quotients that tens in this may stand under Units in the former Product.

倿.

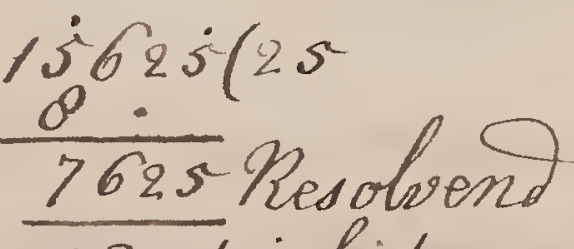

$$
\begin{aligned}
& 12 \text { triples share of- } \\
& 6 \text { triple of the roots } \\
& \text { 125 Divisor } \\
& 60 \text { triplesonare } x \\
& 1 \text { sig triple Rect Xor Xs } \\
& 125 \text { bub of } 5
\end{aligned}
$$

Blevonthly thendraning a Line add the the last Numbers last placed together\& Subtracting the Sum (which is called the ablatiuim) from the Resolvent sit don m the Remainder( if any) in order underneath as in common Subtraction. 


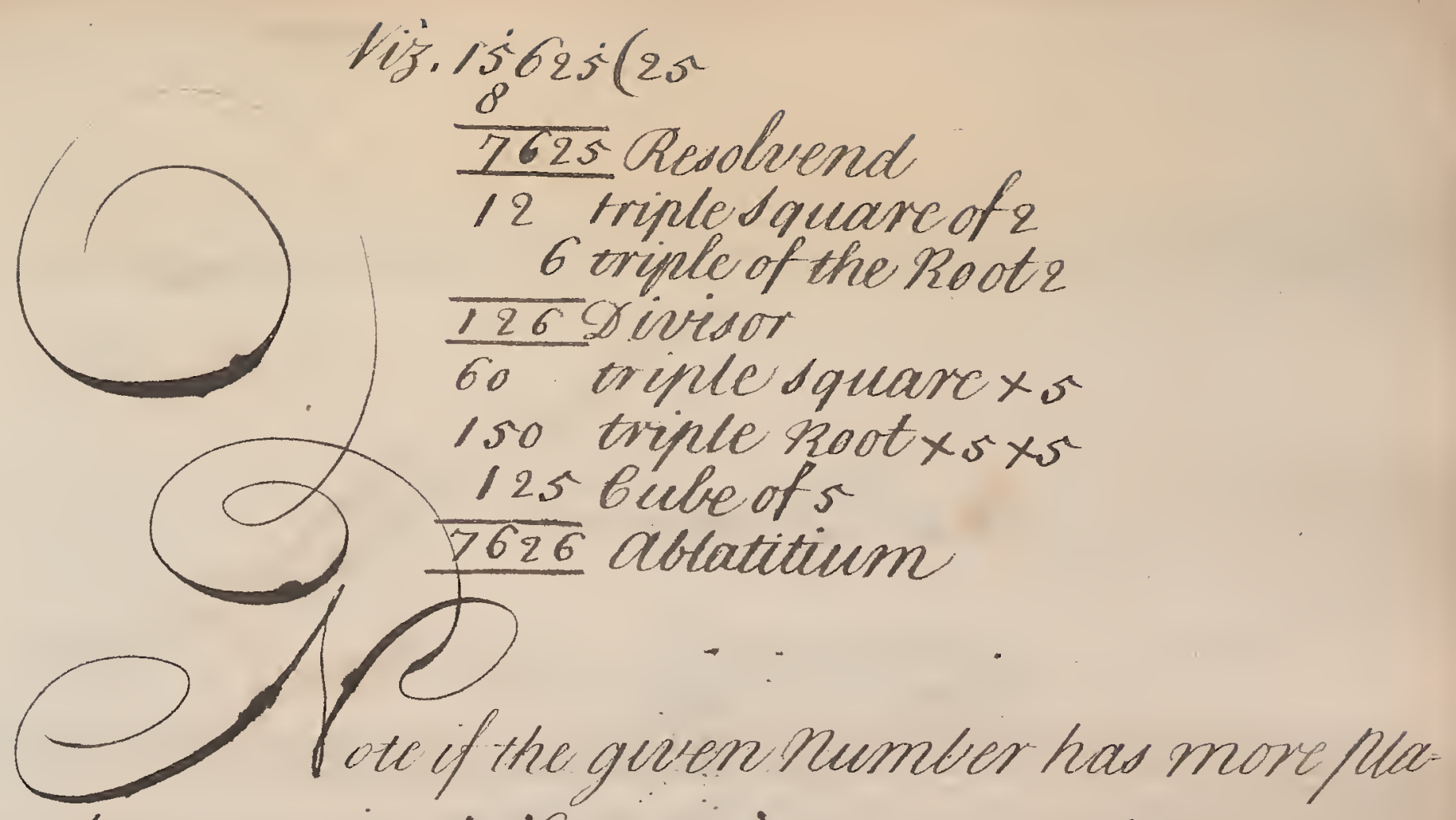

- Us the next single bul mist be brought don n to the last Remainder, for a non Resolvent\& thetwork

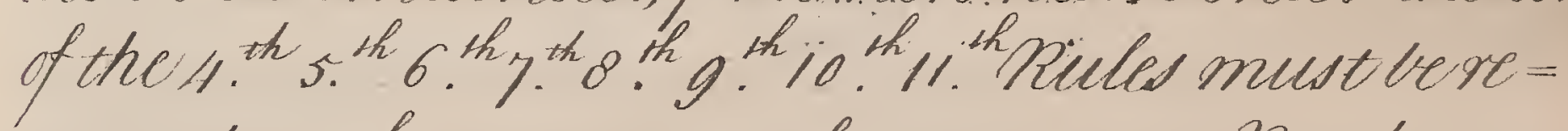
"pleated as often as you so form a non Resolvents shf the ablatitium is greater than the Resolvent the work is false which must le sectify'd by plat sing a lesser figure in the Quotient. 
A Lineal Explanation.

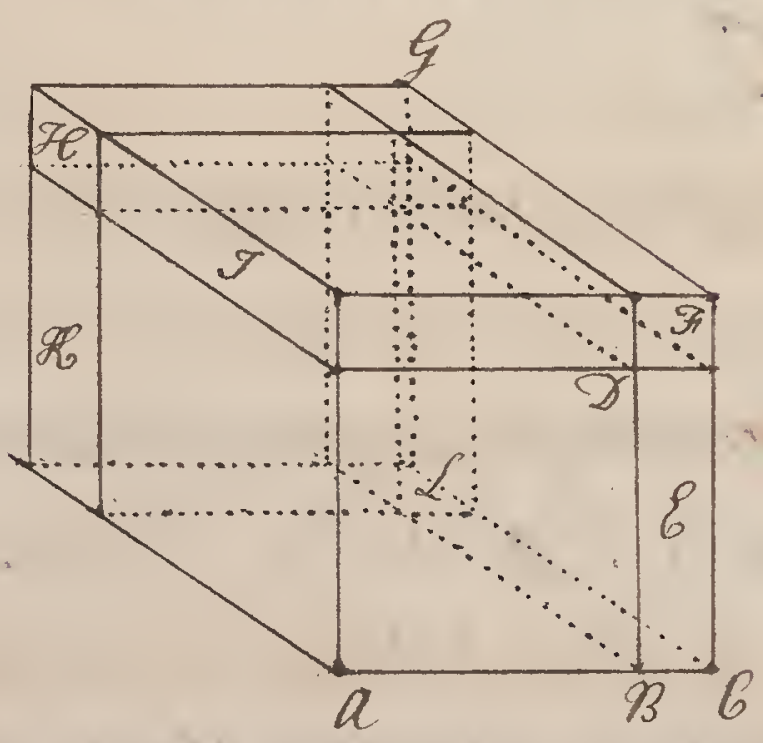

15625 Gube $a y$

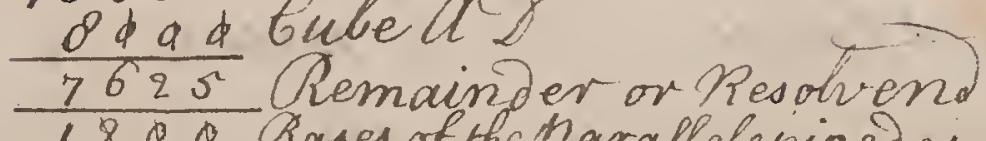

$1 \& Q$ Sases of the harallelepinedes $T$ H 6

$\sigma_{0}$ Height of havallelepipedes $\mathcal{F} \mathscr{L}$

$\frac{1260}{6218}$ Divior

GP Gontent of harallelepipzeoes 9 RE

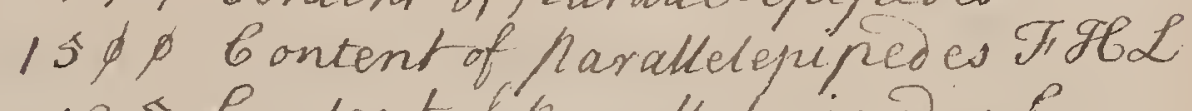
$\frac{125}{2625}$ Gotent of havaluelepipedes $\&$ 
o prove Evolutions or Extractions of the bub Root.

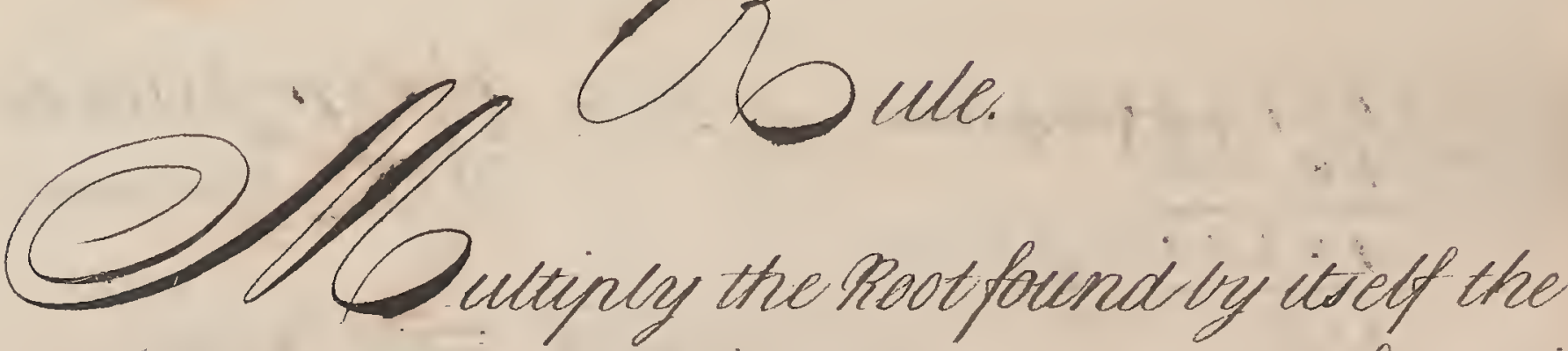

product multiply'd again by the Root to sokich adding the Remainder if any stich if done night noil produce the

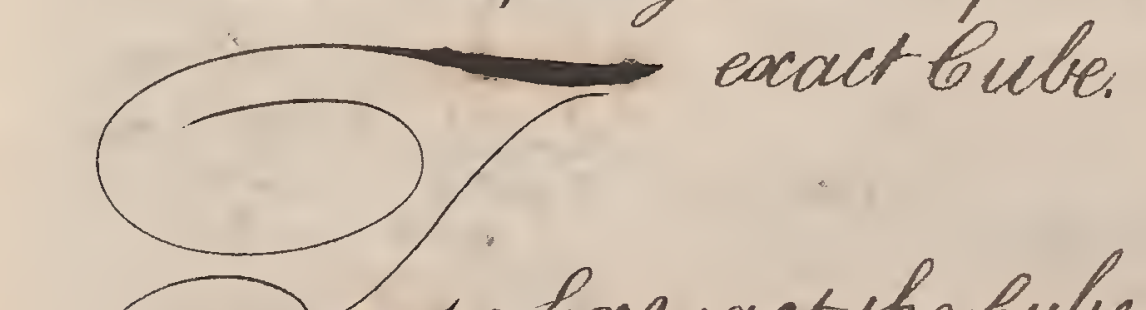

boctract the buber Rest of al lulgarstraction of the Numerator for the Numerator of the Root s the Gull Root of the Denominator for he Denomina. tor of the Root.

But if incommensurable reduce it to aScimols extract the bubenoot thereof. 
What's the Rube Root. What's the bube Root of of 262144 ?

$$
\text { - we62y125? }
$$

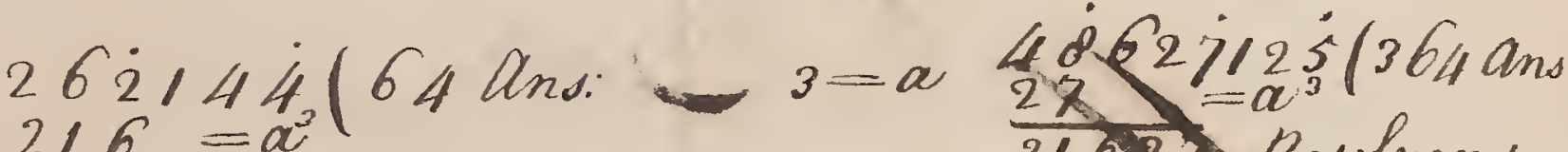
$6=a_{2} 16=a^{2}$

$$
46144 \text { Refohend }
$$

$4=b \frac{1.8}{1098}=3 a$

$$
\begin{aligned}
432 & =3 a^{2} b \\
280 & =3 a b^{2} \\
64 & =b^{3}
\end{aligned}
$$$$
6=b \frac{a}{\frac{279}{162}=3 a^{2} b}
$$$$
324=3 a b^{2}
$$$$
216=b^{3}
$$

19656 Ablatitium

1921125 Resoluend

46144

$36=a \frac{9280}{300}=3 a$ $108=3 a$

$$
4=b \frac{\frac{199 d^{2} \text { Divisor }}{352}=3 a^{2} b}{1,2 a=3 b^{2}}
$$$$
\frac{64}{256}
$$$$
\frac{304}{4096}
$$$$
\frac{64}{16304}
$$

$\frac{24576}{262144}$ lioof. 
What's the Gulve Koot of 13'gos. to Chree Decimal pllaces in the Root?

$$
\begin{aligned}
& 2=a \frac{137900000000000(23.904 / 1 \text { ms: }}{5790^{\circ}} \\
& 3=b \quad \frac{190}{12}=3 a^{2} \text {. Resolvend } \\
& \frac{6}{1269}=3 a \\
& 36=3 a^{2} b \text {. } \\
& 54=3 a b^{2} \\
& \frac{.27}{4167}=\mathbb{C}^{3} \\
& 23=a \frac{\frac{4167 \text { ablatitium st }}{1631000}}{1587}=3 \text {. } \\
& \frac{6 g}{159}=3 a \\
& 558 g=3 a b^{2} \\
& 729=b^{3} \\
& 23.9=a \frac{\frac{1404919 \text { Nblatium 2nd }}{146081000 \text { Resolvend } 3 .}}{171363} \\
& 0=b \quad \frac{717}{17143479 a} \\
& \frac{1714347}{1370904}=3 a^{2} b \\
& 45088=3 a b^{2} \\
& 512=\ell^{3}
\end{aligned}
$$

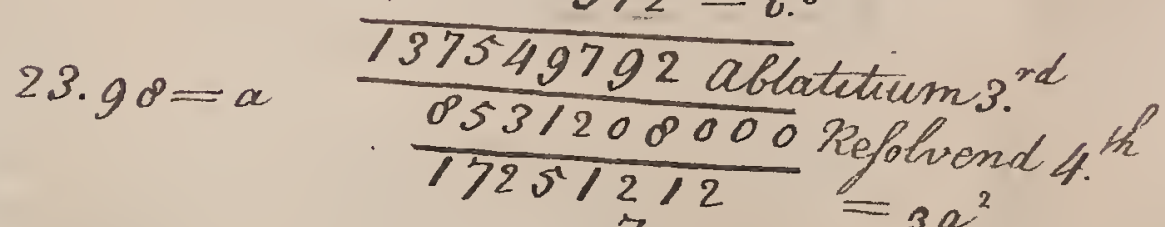

$$
\begin{aligned}
& 115 / 04=3 a^{2} b \\
& 64=b^{3}
\end{aligned}
$$

6901635904 chlatition 4 th $^{\text {th }}$ 1629572096 Refolvend sth 


$$
\begin{array}{r}
23.904 \\
\frac{23904}{95936} \\
191072 \\
215056 \\
79952 \\
479605 \\
575232256 \\
230093904 \\
4601850040 \\
5177090304 \\
1725696768 \\
115046512 \\
1629572096 \\
13798.000000000 \\
\hline
\end{array}
$$

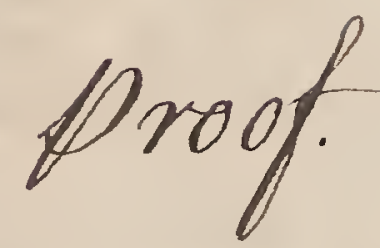

What's the Gube Root of sig?

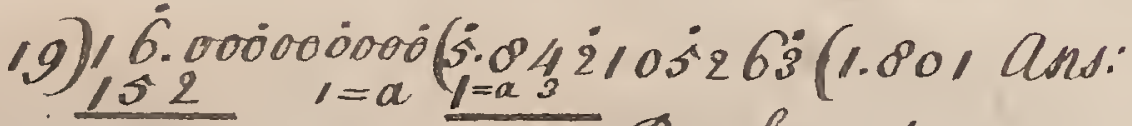

$$
\begin{aligned}
& \frac{76}{40} \quad \frac{4042}{3=3 x^{2}}=3 \text { Rend }
\end{aligned}
$$

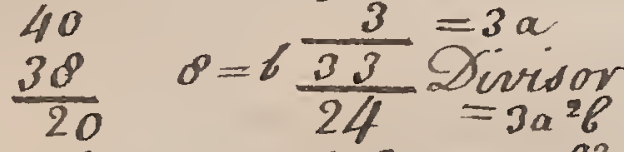

$$
\begin{aligned}
& \begin{array}{ll}
19 & 192=3 a b^{2} \\
100 & 512=b^{3}
\end{array} \\
& \frac{95}{50} \quad 10032 \text { ablatituin } \\
& 30 \cdot 100=a \frac{10105263}{97200}=3 a^{2} \text { Rend } \\
& \frac{30}{120} \quad 540=3 a \\
& \frac{114}{60} 1-b \overline{972540 D \text { Drsor }} \\
& \frac{57}{3} \\
& \frac{97200}{9720}=3 x^{2} b \\
& 540=3 a b^{2} \\
& 9725401 \text { ablatitium }
\end{aligned}
$$

1.801 $\frac{1.801}{1801}$ 144080 $\frac{1801}{3.243601}$ $\frac{1.001}{3243601}$ $259400^{\circ} 080$ 3243601 $\frac{399062}{5042105263 \text { proof }}$ 
What's the bube Root of $\frac{91}{37}$ ?

37)

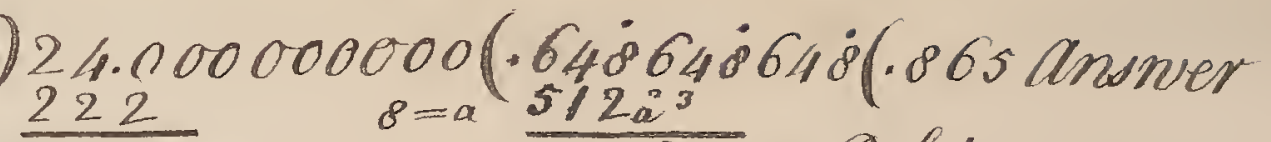

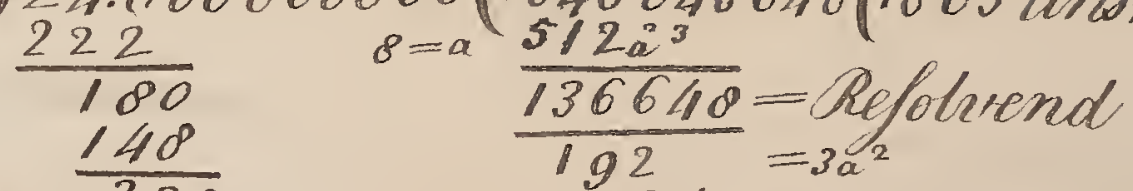

$$
\begin{aligned}
& \begin{aligned}
\frac{148}{320} \\
\frac{296}{240} \\
222
\end{aligned} \quad 6=b \frac{194}{\frac{1944}{1152}}=\text { Divisor } \\
& \frac{222}{100} \\
& \frac{140^{\circ}}{320} \\
& \begin{aligned}
\frac{216}{320} & =b^{3} \\
\frac{296}{24} & 86=\frac{124056}{12592640}=\text { ablitium }
\end{aligned} \\
& 86=\frac{a 12592640^{\circ}}{2210^{\circ}}=\text { Resolvend } \\
& s=b \frac{250}{\frac{22138}{110940}}=3 a \text { Divisor } \\
& 6450=3 a b^{2} \\
& 125=b^{3} \\
& \frac{11158625}{1434023} \text { Resolvend }
\end{aligned}
$$

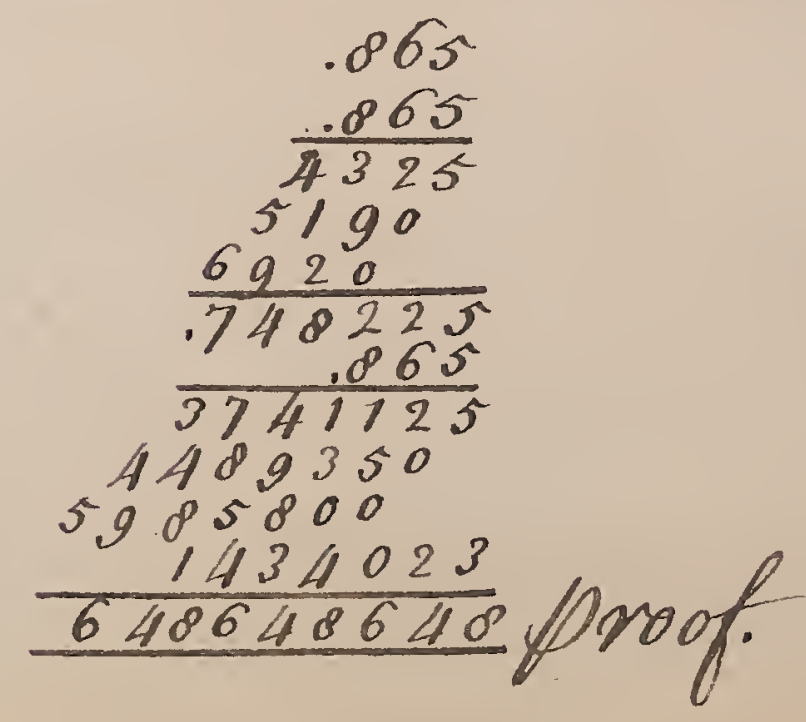




1." Feet \& Inches by Feer \& Snches.

Let 7 Greet g Snchus lic muliplyed by s. Feet 6 J nches?

Feet In:

")"

$30^{0} 9$ Purts:

$\frac{3: 10: 6}{12: 7: 6}$

Let 73 Feet 7 Inches be muliylied byg Feetg Inches? Feet In:

$$
\begin{array}{r}
73: 9 \\
9: 9 \\
55: 0 \text { airt } \\
36: 9: 6 \\
10: 4: 9 \\
4: 6: 0 \\
0: 9: 0 \\
\hline 17: 5=3 \\
\hline
\end{array}
$$


Let 9) Feet is Inches be muluiplied by s. Feer io Snches.

$$
\begin{aligned}
& \text { Fiel In: } \\
& 97: 11 \\
& \frac{0: 10}{776: 0} \text { Parts } \\
& 40: 11: 6: \\
& 32: 7: 0 \\
& 4: 0: 0 \\
& 2: 0: 0 \\
& 1: 4110 \\
& 864: 11: 2 \text { ans: }
\end{aligned}
$$

Let 6g Feelg Snches be muluiply'd by 19 Feety Inches.

$$
\begin{array}{r}
\text { Jieet In: } \\
69: 9 \\
19 " 7 \\
\hline 311 " 0 \\
23: 3 . D_{\text {arto }} \\
17 " 5: 3 \\
9 " 6: 0 \\
4: 9: 0 \\
1365: 11: 3 \\
\hline
\end{array}
$$




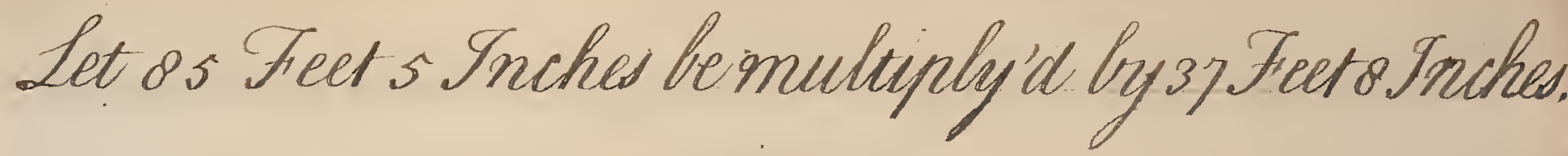

$$
\begin{aligned}
& \text { Feet Sin: } \\
& 85 \div 5 \\
& \frac{3 y: 0}{2595: 0} \\
& 42 " 8=6 \\
& 14 \div 2 \geqslant 10 \\
& 12: 4.0 \\
& \frac{3: 1: 0}{3217: 4: 4}
\end{aligned}
$$

Let $230^{\circ}$ Feet 3 Inches be multiply ed by 40 Feet 11 Inches. 238 "

$$
\begin{aligned}
& \frac{110: 11}{1904: 0} \\
& 952 " 0 \text { Marts } \\
& \begin{array}{l}
59: 6: 9 \\
39: 8: 6
\end{array} \\
& \frac{12: 0: 0}{11654: 4: 9} \text { answer }
\end{aligned}
$$


Muliuly 257 Feel g Snches by S A Feet II Tnches?

$$
\begin{aligned}
& \text { Ticet Inches } \\
& 257 " 9 \\
& 54: 11 \\
& \hline 1028: 0 \\
& 128500 \text { Oarts } \\
& 128: 10: 6 \\
& 64 " 5: 3 \\
& 42: 11 " 6 \\
& 27: 0: 0 \\
& 13: 6: 0 \\
& 14154: 9: 3 \text { ans: } \\
& \hline
\end{aligned}
$$

2. Feet Inchess flarts inte Fet Inches \& Parts.

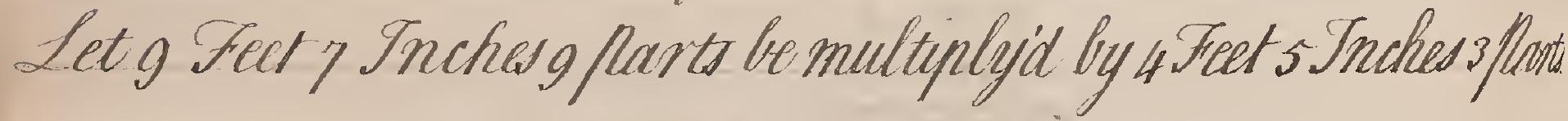
Feet Snches Parts

$$
\begin{aligned}
& 9: 7: 9 \\
& 4: 5 \div 3 \\
& 30: 7 \% 0 \text { Seconds } \\
& 4 \text { " } 0 \text { "2" } 9 \text { Thiords } \\
& 2-4: 11: 3 \\
& \text { 42"9"7"0:3 Ans: }
\end{aligned}
$$




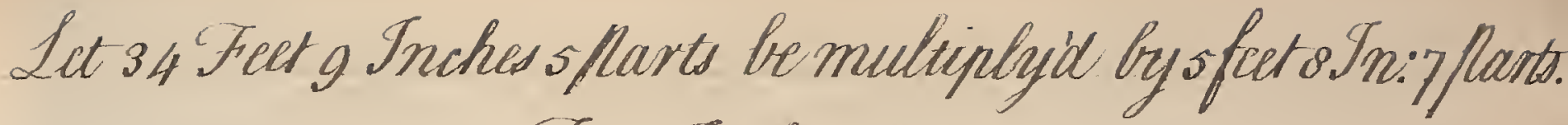

Feet Incheas Parts

$$
\begin{aligned}
& \begin{array}{l}
34: 9=5 \\
5 " 8=2 \\
\hline 170 " 0 ; 0
\end{array} \\
& 17: 4 . " \infty=6 \\
& 5 " 9 " 6 " 10 \text { Thirds } \\
& 1=5 " 4=0,6 \\
& \text { 2: } 2: 6=10: 9 \mathrm{~s} \\
& 2=6: 0: 9=5 \\
& 1=3 ; 0=0=0 \\
& 0: 1 ; 8 ; 0=0 \\
& \frac{0: 0: 5: 0: 0}{198 ; 9: 7: 9: 11} \text { Ans. }
\end{aligned}
$$

Let rng Feet o Inchesso plarts be multiplied by 37 Feet s. Tnches 11 flarts. Feer Tnches parts

$$
\begin{aligned}
& \begin{array}{c}
219 ; 8,10 \\
37: 5: 11 \\
1533: 0=0
\end{array} \\
& 657 " 0 " 0 \text { deconds } \\
& 73 " 2 " 11 " 4 \\
& 10: 340: 10 \\
& 9 \text { " " 10: 5 Thirds } \\
& 4 \text { " 6" 11:2"6 } \\
& 3: 0 ; 7 ; 5: 0 \\
& \begin{array}{l}
18: 6: 5: 0: 0: 0 \\
6: 2: 0 ; 0: 0
\end{array} \\
& 2 \text {; } 0 \text { " } 8 \text { " } 0 \\
& \begin{array}{r}
0: 6 " 6 " 2 " 0 " 0 \\
\hline 8230 " 6 \text { " } 11 " 3: 2 \\
\hline
\end{array}
\end{aligned}
$$


A Sable of the Decimal Marts of a Foot.

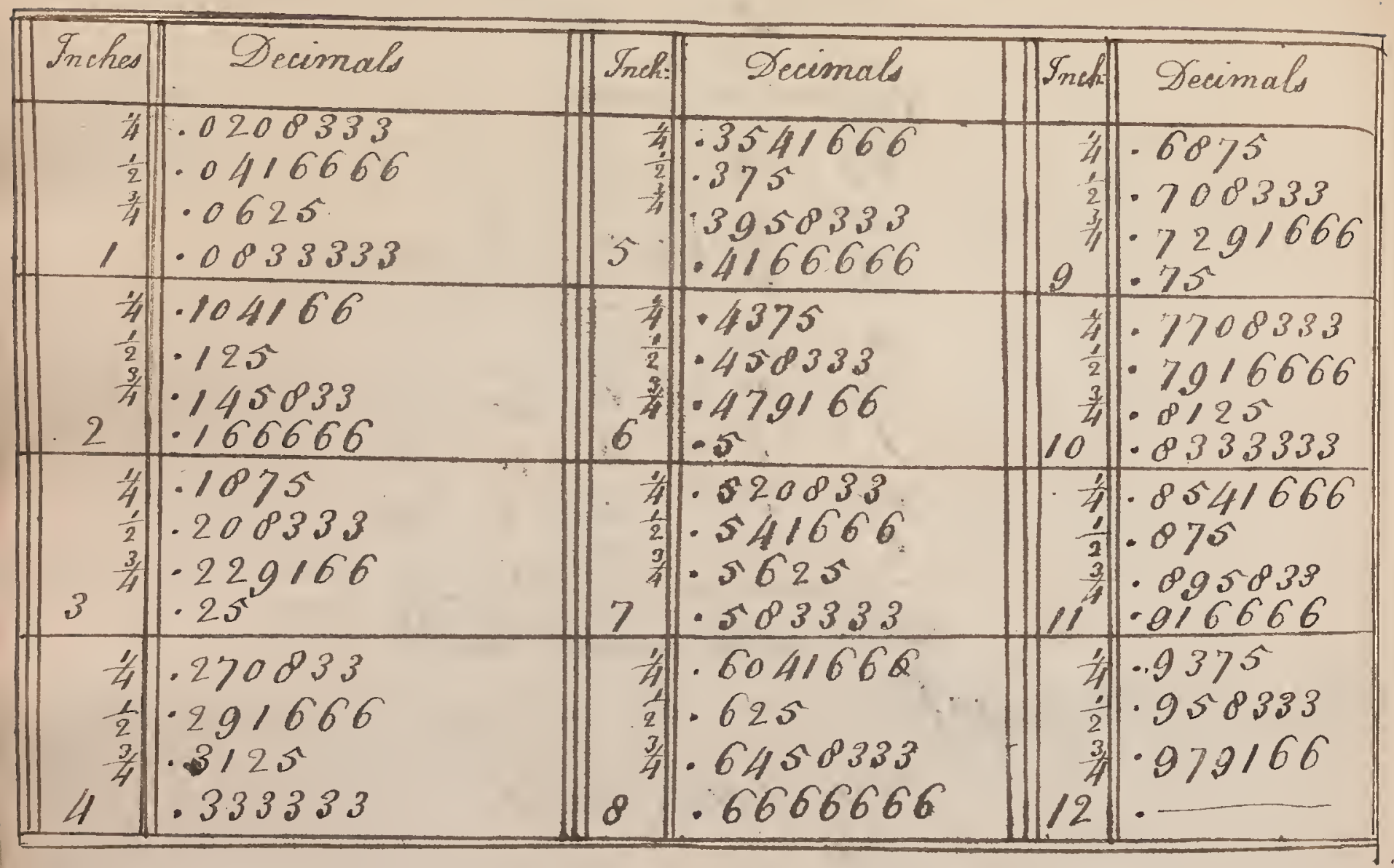


Supnofe a Plane be g feet 10. Inches sin Length\& o Feer $8 \%$ Inches in $73 r e a d t h$, Zuare the fonient or C Irea?

$$
\begin{aligned}
& \text { Teet } \\
& \text { 9.095033 } \\
& \frac{96685}{19479165} \\
& 69,270031 \\
& 9374998^{\circ} \\
& \text { Foet } \frac{79,66664}{85.9700491075}
\end{aligned}
$$

Snchow $11 . \overline{6405902500}$

Canto $7 . \frac{12}{6870830000}$

$$
\text { Sec: } 8 . \frac{12}{2449960000}
$$

$$
\text { Ther } 2 \frac{12}{9399920000}
$$

Feet Frches flarts

$$
\begin{aligned}
& 9=10 \text { " } \\
& \begin{array}{lll}
9: 8=3 \\
\hline 79=2: 0 \\
\hline
\end{array} \\
& 6 \text { " 7" } 2 \text { "0 Thirds } \\
& \begin{array}{r}
0: 2: 5: \infty: 3 \\
\hline 5: 11: 7: 0=3
\end{array}
\end{aligned}
$$

flroof $8511: 7: 0=3$ 


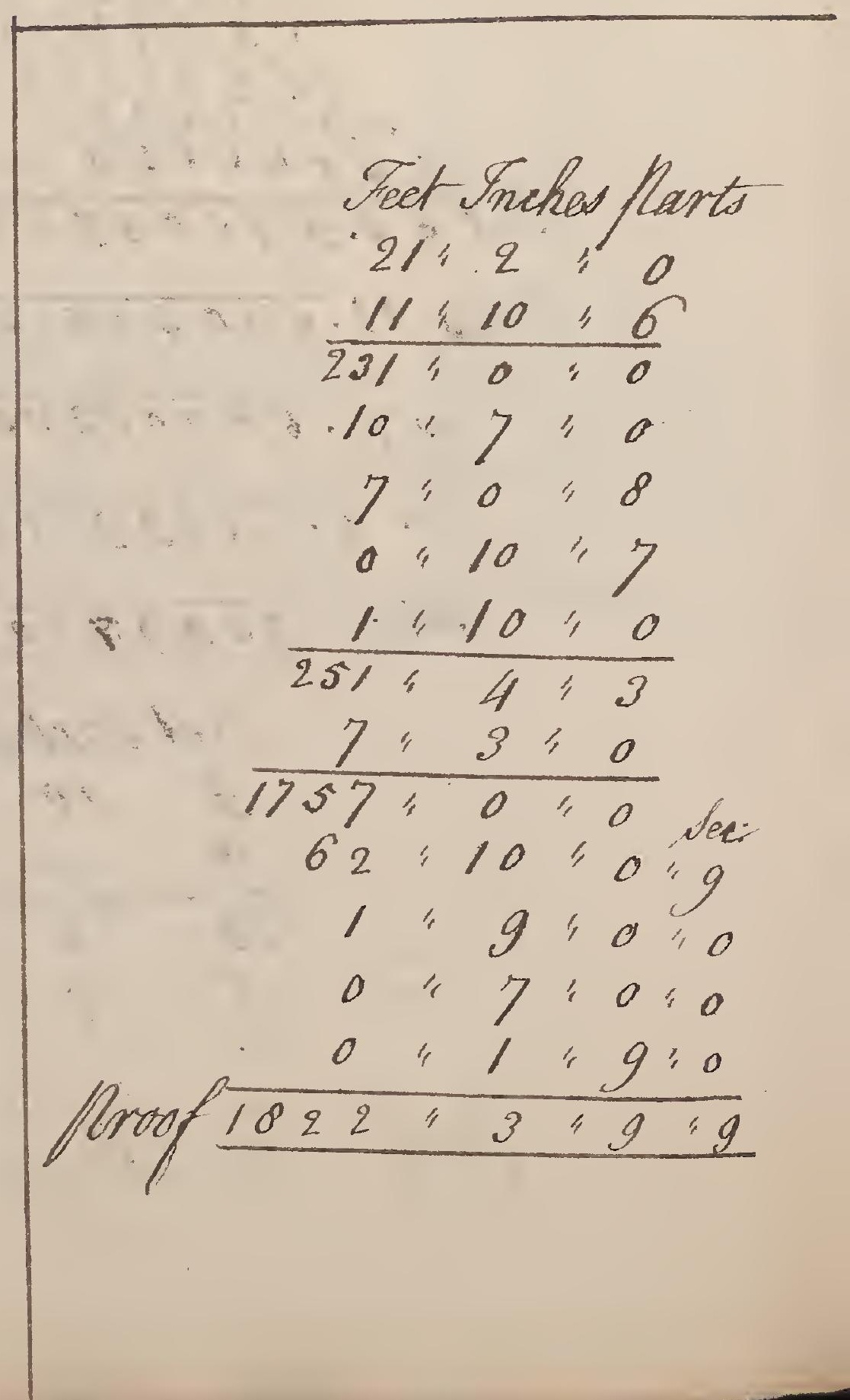


What Number of solid Feet is in a fellar 21 Feet 2 Snches long, "Ifect ro. Inches: broad,s y Seet so Suches decp?

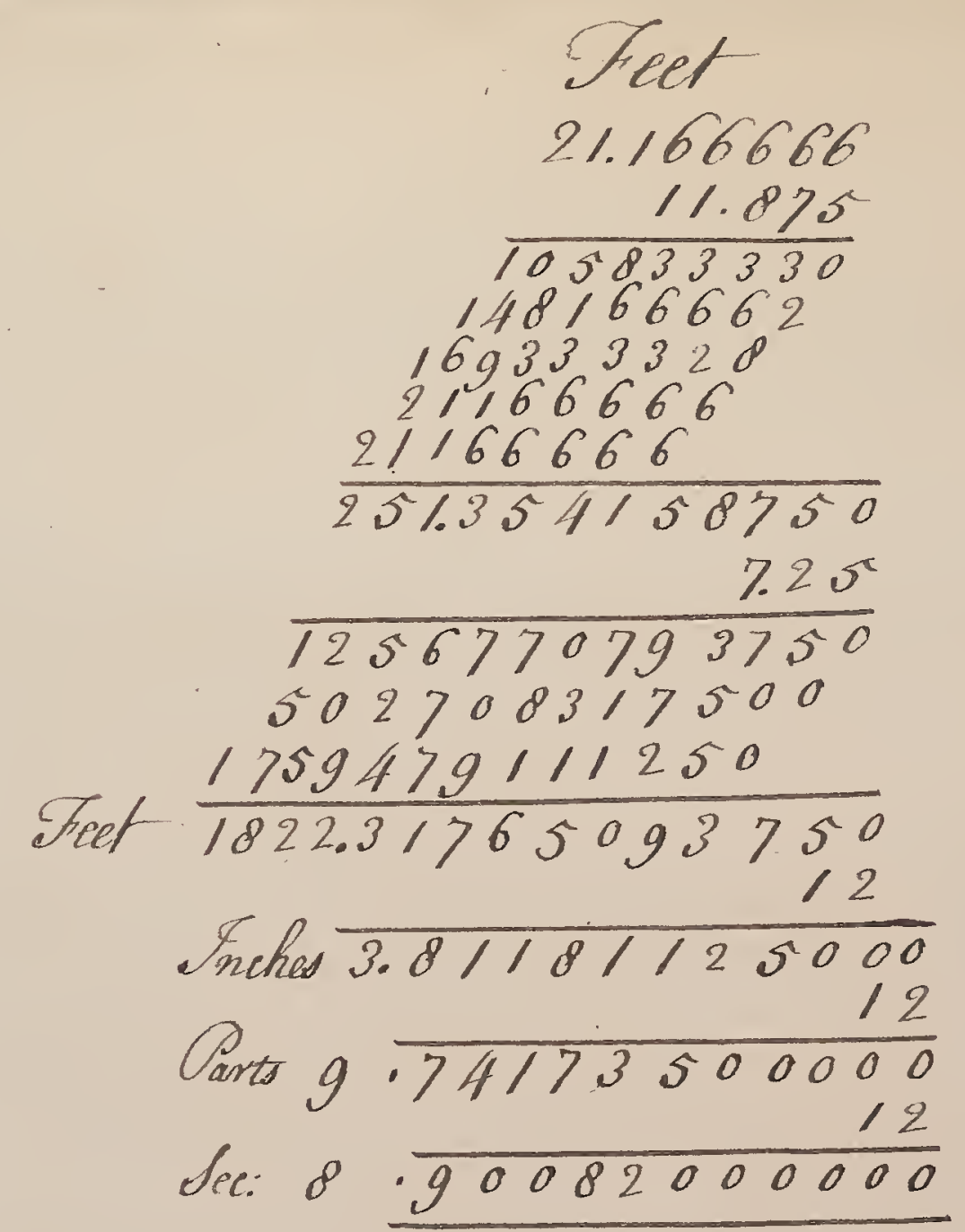

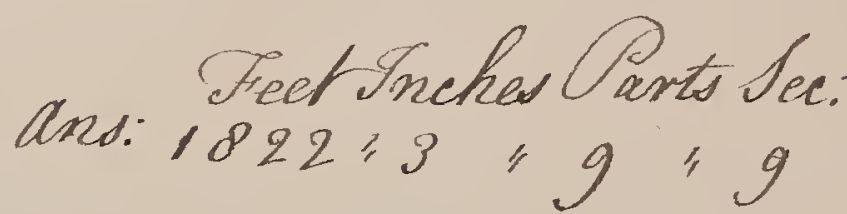





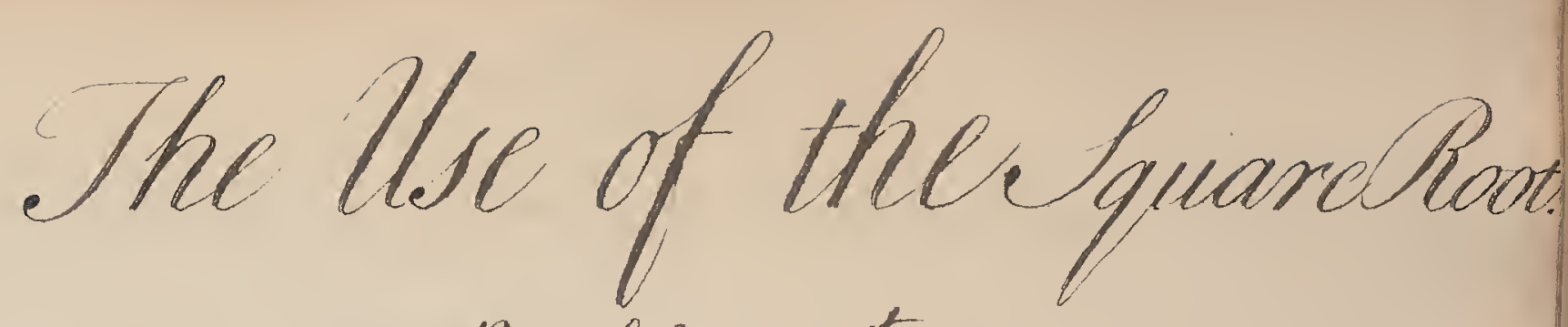

\section{problemi.}

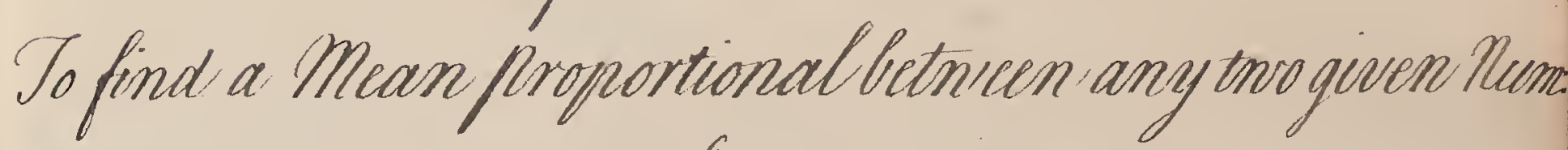

\section{Rule.}

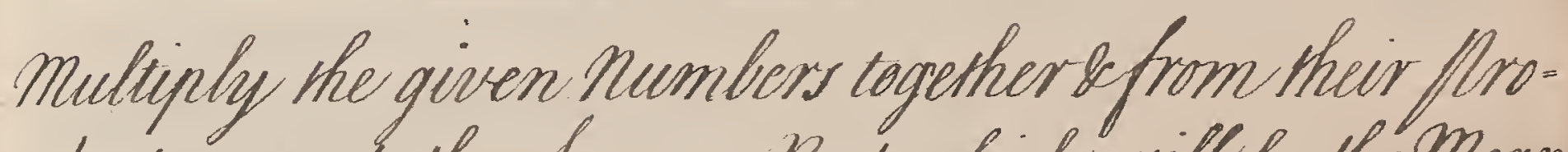
- duct eatract the Square Root which will be the Mean proportional sought.

Let the given Mumbers ber2 \& 48 Whats the Mean proportional betwreen them?

$$
\begin{aligned}
& 48 \\
& 12 \\
& \overline{5 y \dot{6}} \text { (24 Ansoner } \\
& \frac{4}{4 4 \longdiv { 1 7 6 }} \\
& 176
\end{aligned}
$$





\section{Problem. 2.}

So find the Side of a Square whose trea shall be equal to any given Superficies.

\section{Rule.}

bxtract the Square Root from the fontent of any gi= ven Superficies which Root nillle the Side of the square sought adequate thercto.

Let the fontent of ar $\left\{\begin{array}{l}\text { havallelogram } \\ \text { Rhombus }\end{array}\right\}$ be $\left\{\begin{array}{l}14 \\ 810\end{array}\right\}$ Squere of $\alpha$ Rhomboides $\operatorname{sig}$ adequate Regular flotygon) (964) thereto?

$$
\text { 2. }
$$




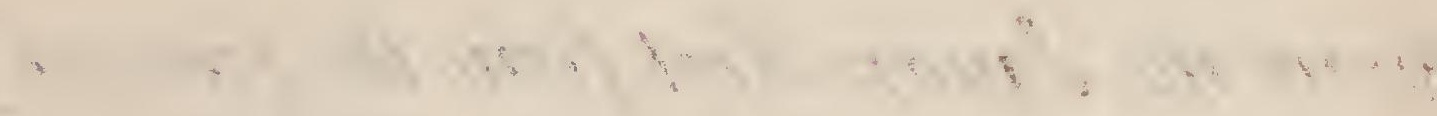

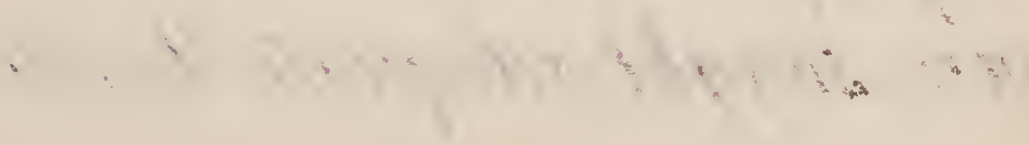

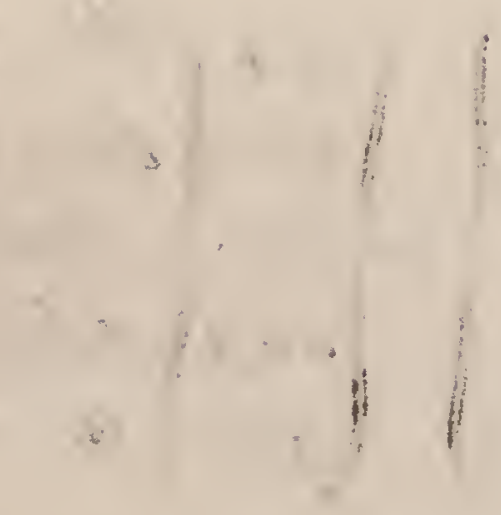

*

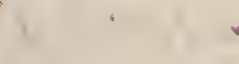




$$
\begin{aligned}
& 468.000000(21.633 \\
& \frac{8}{8} 10.00000000(28.4601
\end{aligned}
$$

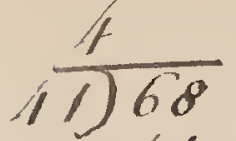

$$
\begin{aligned}
& 180 \frac{4}{\frac{8}{410}} \\
& 4 2 6 \longdiv { 2 7 0 0 } \\
& 5 6 4 \longdiv { 2 6 0 0 } \\
& 4 3 2 3 \longdiv { 1 4 4 0 0 } \\
& 5 6 8 6 \longdiv { \frac { 2 2 5 6 } { 3 4 4 0 0 } } \\
& \begin{array}{r}
5686) \\
34416 \\
\hline 46900
\end{array}
\end{aligned}
$$

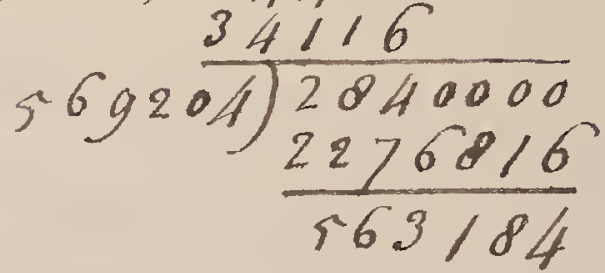

$$
\begin{aligned}
& \begin{array}{r}
4 3 2 6 3 \longdiv { 1 4 3 1 0 0 } \\
\frac{129789}{13311}
\end{array}
\end{aligned}
$$



Problem 3. ${ }^{\text {rd }}$

Thawing any tho sides of a rectangled Triangle to find the third side.

Rule

If you have the trio shortest sides given to find the Loypothenuse or longest side: From the Sum of the Squares of the trio fides extract the Square Root which is the Length of the Boypothenuse.

Let the Base or Breadth of a Ditch be 40 Yards \& the perpendicular or Altitude of the Wall be 30 yards what Length will the Hypothenuse or scaling Ladder be?

$$
\begin{aligned}
& \begin{array}{rr}
30 & 40 \\
30 & 40 \\
\hline 900 & \frac{400}{1600}
\end{array}
\end{aligned}
$$

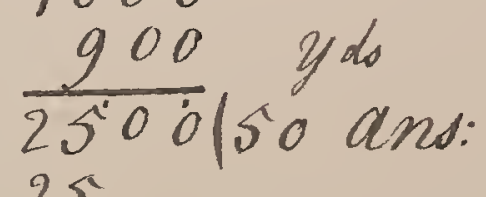

$$
\begin{aligned}
& \frac{25}{00}
\end{aligned}
$$

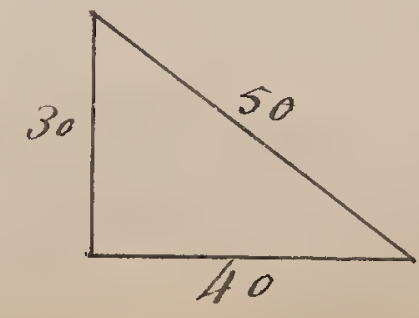





\section{probtem $4 .^{\text {th }}$}

There is a Sower about nhich is a Mloat $40^{\circ}$ Fect widels scaling Ladder bo Feet longwhich will reach from the outside of the Moat to the ten of a Mall nithin the said Moat. Demand the alu - tude of the said Wall above the Water.

\section{Cuele.}

From the Fbypothenuse squar'd deduct the Base squard the square Root of which Remain - der is the Alitude or perpendicular required. 。

given $\left\{\begin{array}{l}\text { Base } 48 \text { Treet } \\ \text { Bypothenuse } 60 \text { Feet }\end{array}\right\}$ io find the Altitude?

$$
\begin{array}{cc}
48 & 60 \\
\frac{48}{384} & \frac{60}{3600} \\
\frac{192}{2304} & \frac{2304}{1296(36} \text { Fret } \\
\hline & \frac{969396}{396} \\
\hline
\end{array}
$$

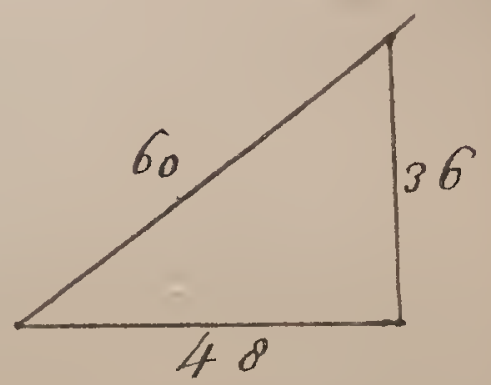



problem s. th

If the Sbypothenuse be perpendicular were give= ven to find the Base.

Rule.

From the Square of the Dbypothenuse deduct the Square of the perpendicular the Square Root of which Remainder is the Base requird.

given $\left\{\begin{array}{l}\text { Hoypothenuse bo feet } \\ \text { perpendicular } 36 \text { feet }\end{array}\right\}$ to find the Base.

$$
\begin{aligned}
& \begin{array}{cr}
60 & 36 \\
\frac{60}{3600} & \frac{36}{216} \\
\frac{1296}{230496} & \frac{100}{1296} \\
\hline 16 &
\end{array} \\
& 8016.4
\end{aligned}
$$

704

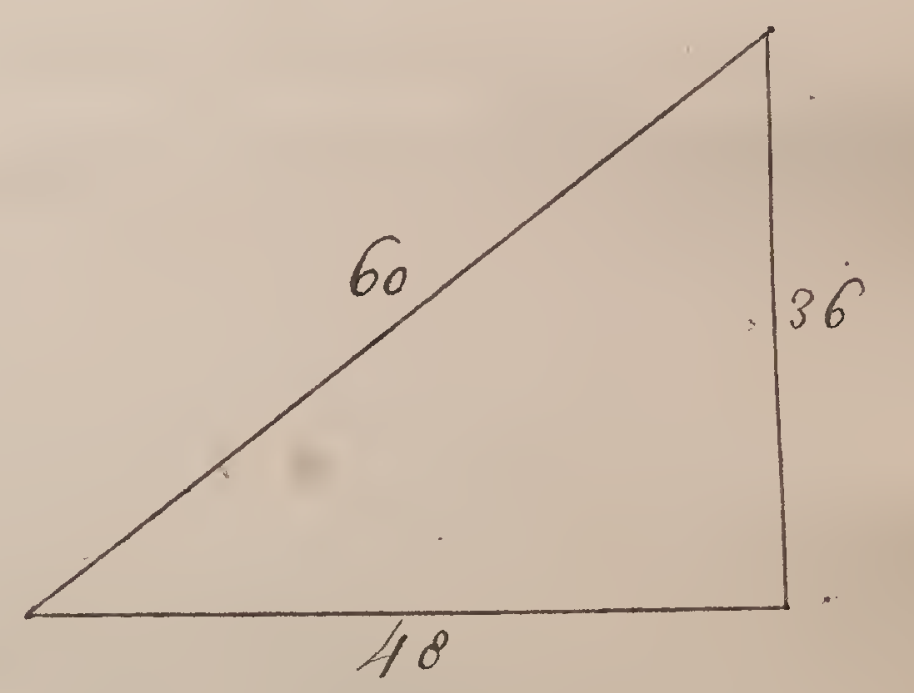



A bompany of men drinking together the Rec= koning came to 6Sodit. IDemand hon many nere in bompany \& hon much each man spent?

$$
\begin{aligned}
& 6=2 \\
& \frac{12}{72} \\
& \frac{4}{2.89(17)} 289(41 / 71 \\
& 189 \quad 119
\end{aligned}
$$

A fompany of Men Drinking together the Recks= - ning came to s. D. Demand hewmany wore in fompany and how mach each man spent?

$$
\begin{aligned}
& \text { I } \\
& \frac{12}{60} \\
& \text { 25) } \frac{1}{140} \frac{15}{90} 4 d .
\end{aligned}
$$



She Use of the Cube Root.

She principal useful Applications whereof are to find out a proportion betricen like Solids as Celebes. Cylinders \& Bubeske.

Brofilem. . st

To find the side of a bubs that shall be equal in Solidity to any given Solid as alylobe, byliner, prism, fore or such like.

Rule

Extract the aube Root of the given fold Portent of any solid body \& it will give the side of the Jube. required.

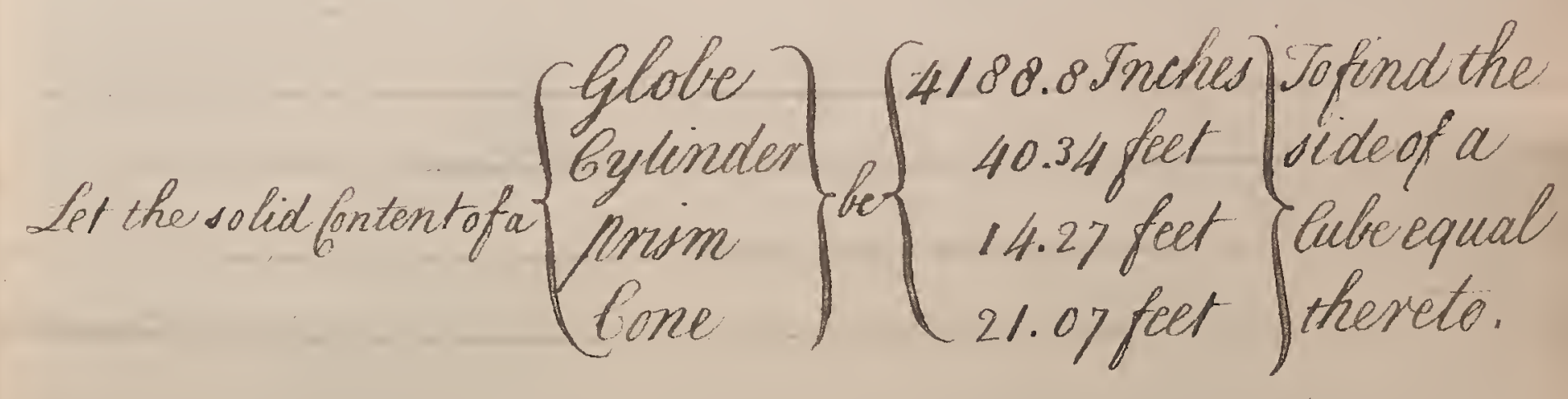





$$
\begin{aligned}
& 1=a \frac{\begin{array}{l}
4188.800000(16.09 \\
\frac{1}{3188}
\end{array} \quad 3=a \frac{27}{13340}=a^{3}}{\frac{27}{3}} \\
& \frac{3188}{3}=3 a^{2} \quad \frac{13340}{27}=3 a^{2} \\
& 6=f \frac{3}{33}=3 a \\
& 4=b \frac{9}{\frac{279}{10 d}}=3 a \\
& 100^{8}=3 a b^{2} \\
& 144=3 a^{b^{2}} \\
& \frac{\frac{216}{3096}=b^{3}}{\frac{92800000}{76800}}=3 a^{2} \quad 34=\frac{\frac{64}{12304}=b^{3}}{\frac{1036000}{3468}}=3 a^{2} \\
& \begin{array}{rlrl}
160=a & \frac{92800}{76 a^{2}} & \frac{348}{34 a^{2}} \\
480 & =3 a & 102 & =3 a
\end{array} \\
& g=b \frac{\frac{480}{768440}}{691200}=3 a x=3 x^{2} b=\frac{102}{34982}=3 a \\
& 38080=3 a b^{2} \\
& 408=3 a b^{2} \\
& 729=b^{3} \\
& \frac{\frac{69509529}{23290421}}{16.09} \\
& 8=\ell^{3} \\
& \frac{697688}{338312} \\
& \begin{array}{r}
3.42 \\
3.42 \\
684 \\
1368 \\
1026 \\
\hline 11.6964 \\
33942 \\
1969896 \\
350992 \\
338312 \\
30.340000 \text { Proof }
\end{array} \\
& \begin{array}{r}
3.42 \\
3.42 \\
684 \\
1368 \\
1026 \\
\hline 11.6964 \\
33942 \\
1969896 \\
350992 \\
338312 \\
30.340000 \text { Proof }
\end{array} \\
& \begin{array}{r}
14481 \\
96540 \\
\frac{1609}{2580881} \\
\frac{1609}{23299929}
\end{array} \\
& 155332860 \\
& 258888 \\
& \frac{232901171}{1188.800000} \text { flory }
\end{aligned}
$$





$$
\begin{aligned}
& 14.2700000(242 \\
& 21.070000(2.76 \\
& 2=a \frac{8}{\frac{6270}{12}}=3 x^{2} \\
& 2=x \\
& \frac{8}{13070}=a^{3} \\
& 4=6 \frac{6}{\frac{126}{48}}=3 a^{2} b \\
& \frac{\overline{13070}}{12}=3 a^{2}
\end{aligned}
$$

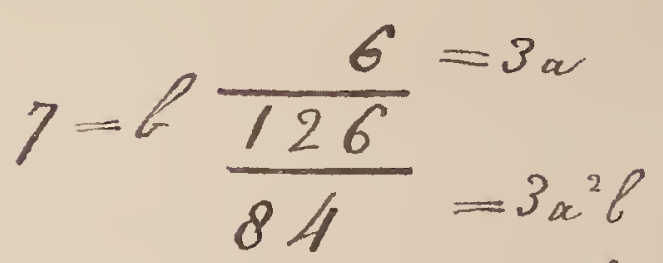

$$
\begin{aligned}
& 96=3 a b^{2} \\
& 294=3 a b_{2} \\
& \frac{64}{5884}=b^{3} \\
& 343=b^{3} \\
& 24=a \frac{446000}{1728=3 a^{2}} \\
& 27=a \frac{\frac{11687000}{2187=3 x^{2}}}{\frac{138}{280}} \\
& 81=3 x \\
& 2=f \frac{\frac{72}{1.7352}}{3456}=3 a^{2} b \\
& 6=b \frac{\frac{21951}{13122}=3 a^{2} b}{8 a b} \\
& 288=3 \times b^{2} \\
& 29 / 6=3 a b^{2} \\
& \frac{\frac{8}{348488}}{97512} \\
& \frac{216}{1341576} \\
& \begin{array}{l}
2.42 \\
2.42 \\
\hline 484
\end{array} \\
& \begin{array}{r}
968 \\
484 \\
\hline 5.8564
\end{array} \\
& 2.76 \\
& \frac{2.76}{1656} \\
& 1932 \\
& \frac{552}{7.6176} \\
& \frac{2.42}{1172128} \\
& \frac{2.76}{457056} \\
& \begin{array}{r}
117128 \\
99712 \\
14.270000 \\
\hline
\end{array} \\
& \begin{array}{r}
533232 \\
152352
\end{array} \\
& \frac{15235424}{21.079000} \text { proof }
\end{aligned}
$$



Problem 2.

Shaving the Diameters Wright of a Bullet to find the Night of another Bullet whole Diameter is given.

sue.

As the full of the Given Bullet's Diameter Ss to its Solidity or Weight

So is the full of the Diameter of any other Bullet To its Weight or Solidity.

If a Bullet of Brags of I Inches Diameter weigh 'th 'What shall a Bullet wright of Brats weigh whose Diameter is 11 Inches?

\begin{tabular}{rr}
8 & 4 \\
$\frac{8}{64}$ & $\frac{4}{16}$ \\
$\frac{8}{512}$ & $\frac{4}{64}$ \\
\cline { 2 - 2 }
\end{tabular}

$$
\begin{aligned}
& \begin{array}{r}
512: 72:: 64 \\
\frac{72}{128}
\end{array} \\
& 5 1 2 \longdiv { 4 6 0 8 1 9 } \frac { 4 0 } { 4 0 } \text {. }
\end{aligned}
$$

4608 



\section{probtem 3.-}

The Side or Root of a bube bing givento find the side of another bube that shall be double, treble, quadruplesec. or quarter, half, \& three quarters, in 2uantity to the given bube. Rule

bube your side given which muliplyed by 2,3 $48 \mathrm{c}$. Ana the bube Reot of the Product is the Side sought.

Suppose a bubical Vigoel whose Side is 12 Inches \&e it is required to find the Side of another Vefselwhich shall contain s times as much?

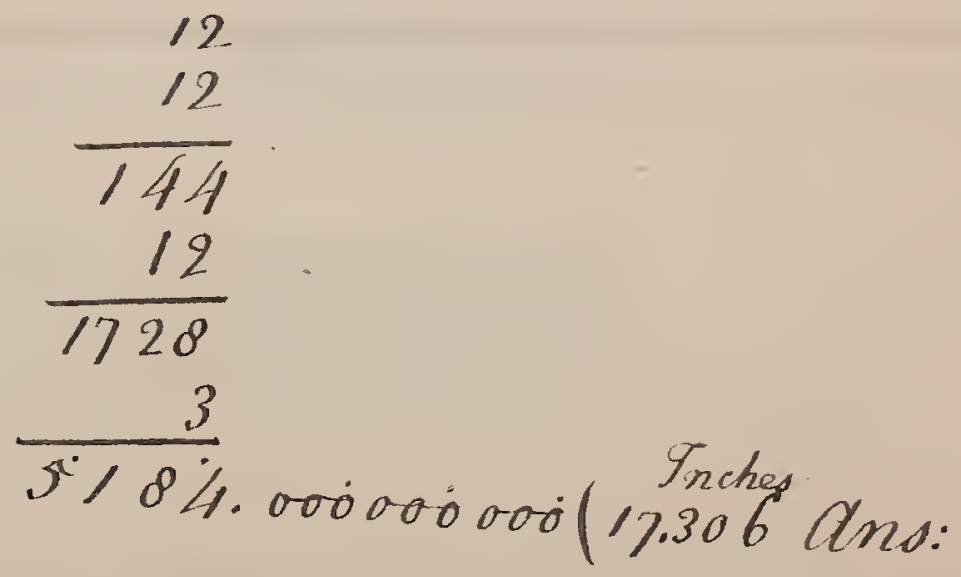





$$
\begin{aligned}
& \text { sis } 4.000000000 \text { (17.306 Ans. } \\
& 1=a \frac{1-a^{3}}{\frac{4184}{3-3 a^{2}}} \\
& 7=b \quad \frac{3}{33}=3 a \\
& 17.306 \\
& \frac{17.306}{103836} \\
& 519180 \\
& 2 \overline{1}=3 x^{2} b \\
& 121142 \\
& 147=3 a b^{2} \\
& \frac{343}{49 / 3}=l_{3} \\
& \frac{17306}{299.497636} \\
& \frac{17.306}{1796985816} \\
& 8984929080 \text {. } \\
& 20961183452
\end{aligned}
$$

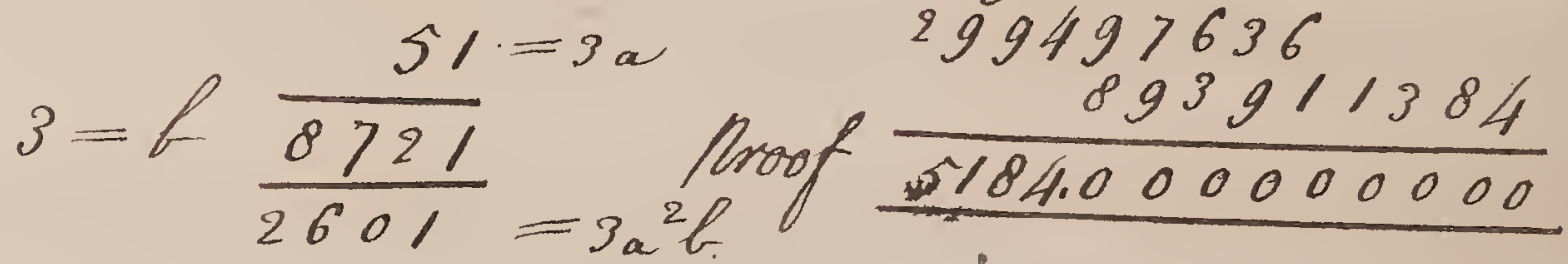

$$
\begin{aligned}
& 45 g=3 a b^{2} \\
& 27=6^{3} \\
& 264717 \\
& 1730=a \frac{6283000000}{8978700}=3 a^{2} \\
& 6=f \quad \frac{5 / 90}{89792190}=3 a \\
& 5 \frac{8872200}{538 a^{2} b} \\
& 186840=3 a b^{2} \\
& 216=63 \\
& \frac{5389088616}{893911384}
\end{aligned}
$$



It is required to find che Side of a Cubical Vlf=

sol that shall contain 's as much as another Cubical Vefsel whose Side is 20 Inches?

$$
\begin{aligned}
& 20 \\
& \frac{20}{400} \\
& 20 \\
& 2000.000000000 \text { (12.59g Answer. } \\
& 1=a \quad \frac{1}{1000}=a^{3} \\
& 2=b \frac{\frac{3}{33}}{6}=3 a \\
& 12=3 a b^{2} \\
& =b_{3} \\
& 12=a \frac{\frac{728}{272000}}{432}=3 a^{2} \\
& s=b-\frac{36}{4356}=3 a \\
& 900=3 a b^{2} \\
& \frac{\frac{125}{225125}=63}{46875000}
\end{aligned}
$$





$$
\begin{aligned}
& 125=a \quad \frac{46875000}{46875}=3 a^{2} \\
& 375^{\circ}=3 a \\
& g=b \frac{\frac{469125}{421875}=3 a^{2} b}{3 a b} \\
& 30375=3 a b^{2} \\
& 729=b^{3} \\
& \overline{42491979} \\
& 1259=a \frac{4383021000}{4755243}=3 a^{2}
\end{aligned}
$$

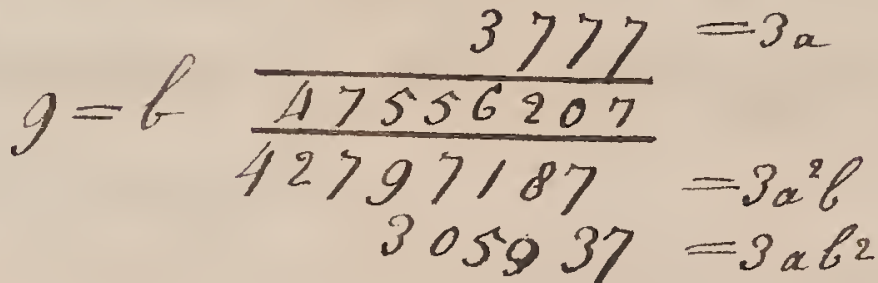

$$
\begin{aligned}
& 72 g=b^{3} \\
& \frac{4282798799}{100242201} \\
& 12.599 \\
& \frac{12.599}{113391} \\
& 113391 \\
& 62995 \\
& \frac{151188}{158.734801} \\
& \frac{12.599}{1428613209} \\
& 1428613209 \\
& \text { Aropt } \\
& \begin{array}{r}
199674005 \\
1904817612 \\
100242201 \\
\hline 2000.000000000
\end{array}
\end{aligned}
$$



Between two given Numbers to find two mean proportionals

\section{cucule}

Multiply the lefs \&xtream by the bubr Root of the Quo= tient of the greater bxtream divided by the lefs the pro duct is theilesser of the two Mean Proportionals which multiplyed by the said bube Root gives the great or Mean fought.

Suppose trivo Propertionals lietrisat 6\& 162 nere to be sought What arc they?

$$
\begin{aligned}
& \frac{6162}{27}(3 \\
& \frac{27}{0}=a^{3}
\end{aligned} \quad \frac{6}{18} \quad \frac{18}{54} \quad 6.18 \cdot 54 \cdot 162
$$





\section{flroblem s.}

The boncave Diameter of 2 Guns biing known togethernith the 2uantity of Gun-ponder fufficient tocharge one to find what svill be suffrient to charge cheosther.

As the bubc of that Diameter nhese Luantity is given $\{$ Ts to che sucantity of Gumponder given

sois the fulc of the Piameter whose 2uantity is requird So its Luantity of Gun-lowder required.

If.43 of a lli of Gum flowder be fufficient to charge a. Gun whofe Goncanc Diameter is 1. S Snch how much Gun-Bowder will suffice to charge a Gun nhese Di: ametor is Inches?

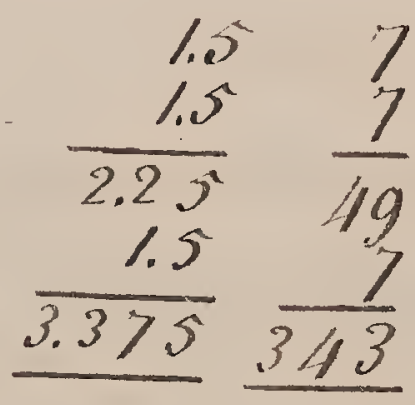

$$
\begin{aligned}
& 3.375: 43: \because 343 \\
& \frac{143}{1029} \\
& 1372^{\circ} \\
& 3 . 3 7 3 \longdiv { 1 4 7 . 4 9 0 0 ( 4 3 . 7 \text { Ans: } } \\
& \frac{13500}{12490} \\
& \frac{10 / 25}{23650} \\
& 23625
\end{aligned}
$$





\section{problem 6.}

The Boncave Diameters of two Guns feing given and the Quantity of a neaker Sort of Gun-Conder sufficient to charge one of them to find how much Gun-flowder of a stron ger Sort (the Proportion of the Strength \& Weaknefs of the gun-fonder being also ginen) nill be sufficient to charge the other feen:

First by Reciprocal Proportion find how much of the stronger Sort of Gun Ponder will lie equivalent in drength nith the given 2 uantity of the weaker fort the work by Dis rect proportion as in the poreceding florblem.

If. 43 lb of Gun-pouder be sufficient to charge a qun

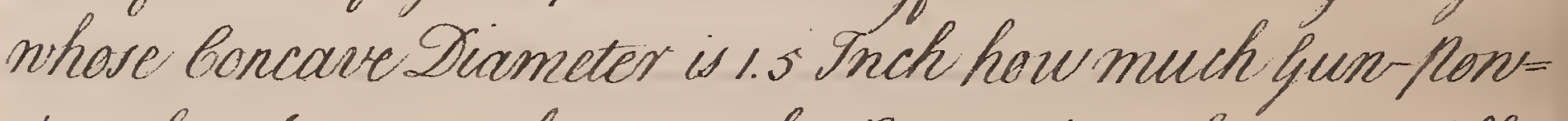
der of a Stronger fort in the floroportion of 5 to 2 nill suffice to charge a fun whose boncaure Diameter is

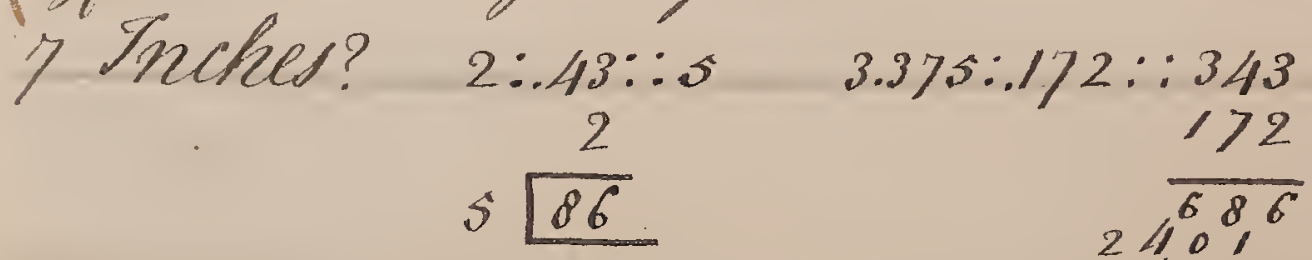

$$
\begin{aligned}
& \begin{array}{c}
1.57 \\
\frac{1.5}{2.25} \frac{7}{49} \\
\frac{1.5}{3.375} \frac{7}{3113}
\end{array} \\
& 3 . 3 7 5 \longdiv { \frac { 3 4 3 } { 5 8 . 9 9 6 0 0 } } \frac { \frac { 3 3 7 5 } { 2 5 2 4 6 } } { \frac { 2 . 3 6 2 . 5 } { 1 3 5 1 0 } }
\end{aligned}
$$

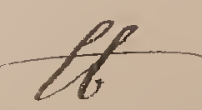










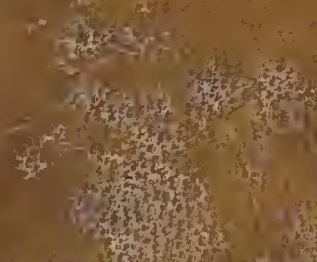

SMITHSONIAN INSTITUTION LIBRARIES

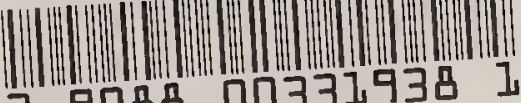

э 9088 B

nmah Arithmetic 